\title{
PdCl $_{2}$-Catalyzed Efficient Transformation of Propargylic Amines to (E)- $\alpha$-Chloroalkylidene- $\beta$-lactams
}

\author{
Shengming Ma*, Bin Wu and Xuefeng Jiang
}

State Key Laboratory of Organometallic Chemistry, Shanghai Institute of Organic Chemistry, Chinese Academy of Sciences, 354 Fenglin Lu,

Shanghai 200032, P. R. China

masm@mail.sioc.ac.cn

Supporting Information

Table of Contents

\begin{tabular}{c|c}
\hline Analytical data for compounds $\mathbf{3}, \mathbf{4}$ and $\mathbf{6}$ & S2 \\
\hline${ }^{1} \mathrm{H}$ NMR and ${ }^{13} \mathrm{C}$ NMR spectra for those compounds & S22 \\
\hline
\end{tabular}




\section{The following compounds were prepared according to Typical Procedure I.}

\section{(1) $N$-(Non-4-yn-3-yl)amine (3b).}

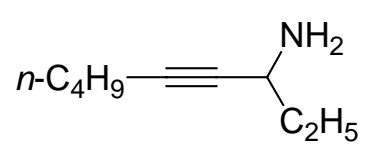

The reaction of $\mathbf{1 b}$ (4.996 g, $35.7 \mathrm{mmol}$ ), phthalimide (5.789 g, $39.4 \mathrm{mmol}), \mathrm{PPh}_{3}$ (10.291 g, 39.3 mmol), and diethyl azodicarboxylate

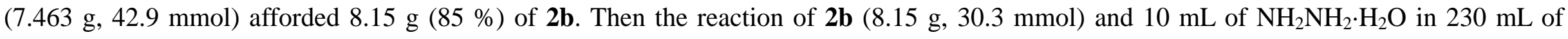
absolute EtOH afforded 2.464 g (58 \%) of 3b: liquid; ${ }^{1} \mathrm{H}$ NMR (300 MHz, $\left.\mathrm{CDCl}_{3}\right) \delta 3.60-3.50$ (m, $\left.1 \mathrm{H}\right), 2.19$ (t, $\left.J=5.9 \mathrm{~Hz}, 2 \mathrm{H}\right), 1.75-1.30$ (m, $8 \mathrm{H}), 0.99(\mathrm{t}, J=7.4 \mathrm{~Hz}, 3 \mathrm{H}), 0.91(\mathrm{t}, J=6.9 \mathrm{~Hz}, 3 \mathrm{H}) ;{ }^{13} \mathrm{C}$ NMR $\left(75.4 \mathrm{MHz}, \mathrm{CDCl}_{3}\right) \delta$ 83.3, 82.5, 50.0, 31.5, 30.9, 21.8, 18.3, 13.5, 10.3; MS (EI) m/z (\%) 139 (M+, 0.10), 110 (100); IR (neat) 3369, 2235, $1595 \mathrm{~cm}^{-1}$; HRMS (MALDI/DHB) calcd for C9 $\mathrm{H}_{18} \mathrm{~N}\left(\mathrm{M}^{+}+1\right)$ 140.1434. Found 140.1439.

(2) N-(2-Methylnon-4-yn-3-yl)amine (3c).<smiles>CCCCCCC#CC(N)[In]CC</smiles> 
The reaction of 1c (6.157 g, $40 \mathrm{mmol})$, phthalimide (6.468 g, 44 mmol), $\mathrm{PPh}_{3}$ (11.528 g, 44 mmol), diethyl azodicarboxylate (8.596 g, 49.4 mmol) afforded $7.571 \mathrm{~g}(67 \%)$ of 2c. Then the reaction of 2c (7.563 g, $26.7 \mathrm{mmol})$ and $10 \mathrm{~mL}$ of $\mathrm{NH}_{2} \mathrm{NH}_{2} \cdot \mathrm{H}_{2} \mathrm{O}$ in $250 \mathrm{~mL}$ of absolute EtOH afforded 2.172 g (53 \%) of 3c: liquid; ${ }^{1} \mathrm{H}$ NMR (300 MHz, $\left.\mathrm{CDCl}_{3}\right) \delta 3.44-3.35$ (m, $\left.1 \mathrm{H}\right), 2.19$ (dt, $J=2.0$ and $\left.6.8 \mathrm{~Hz}, 2 \mathrm{H}\right), 1.82-1.65$ (m, $1 \mathrm{H}$ ), 1.60-1.31 (m, $6 \mathrm{H}), 0.97$ (d, $J=6.6 \mathrm{~Hz}, 6 \mathrm{H}), 0.91$ (t, $J=7.1 \mathrm{~Hz}, 3 \mathrm{H}) ;{ }^{13} \mathrm{C}$ NMR $\left(75.4 \mathrm{MHz}, \mathrm{CDCl}_{3}\right) \delta$ 83.2, 81.8, 49.6, 34.5, 30.9, 21.8, 19.1, 18.2, 17.5, 13.5; MS (EI) m/z (\%) $153\left(\mathrm{M}^{+}, 0.19\right), 110$ (100); IR (neat) 3376, 2237, $1598 \mathrm{~cm}^{-1}$; HRMS (MALDI/DHB) calcd for $\mathrm{C}_{10} \mathrm{H}_{20} \mathrm{~N}\left(\mathrm{M}^{+}+1\right)$ 154.1590. Found 154.1596.

\section{(3) N-(1-Cyclohexyl-2-heptynyl)amine (3d).}

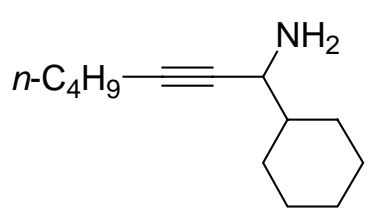

The reaction of 1d (5.038 g, 26 mmol), phthalimide (4.224 g, 28.7 mmol), $\mathrm{PPh}_{3}$ (7.498 g, 28.6 mmol), diethyl azodicarboxylate (5.431 g, $31.2 \mathrm{mmol}$ ) afforded $5.141 \mathrm{~g}(61 \%)$ of $\mathbf{2 d}$. Then the reaction of $\mathbf{2 d}(5.129 \mathrm{~g}, 15.88 \mathrm{mmol})$ and $8 \mathrm{~mL}$ of $\mathrm{NH}_{2} \mathrm{NH}_{2} \cdot \mathrm{H}_{2} \mathrm{O}$ in $230 \mathrm{~mL}$ of absolute EtOH afforded 2.451 g (80 \%) of 3d: liquid; ${ }^{1} \mathrm{H}$ NMR (300 MHz, $\left.\mathrm{CDCl}_{3}\right) \delta$ 3.43-3.30 (m, $\left.1 \mathrm{H}\right), 2.18$ (dt, $\left.J=1.7 \mathrm{and} 6.3 \mathrm{~Hz}, 2 \mathrm{H}\right), 1.91-1.56$ (m, 
$5 \mathrm{H}), 1.55-0.98$ (m, $12 \mathrm{H}), 0.91(\mathrm{t}, J=7.2 \mathrm{~Hz}, 3 \mathrm{H}) ;{ }^{13} \mathrm{C}$ NMR (75.4 MHz, $\left.\mathrm{CDCl}_{3}\right) \delta$ 83.2, 82.3, 48.9, 44.4, 31.0, 29.6, 28.2, 26.4, 26.1, 26.0, 21.9, 18.3, 13.5; MS (EI) m/z (\%) 194 (M+1, 12.48), 110 (100); IR (neat) 3374, 2228, $1595 \mathrm{~cm}^{-1}$; HRMS (MALDI/DHB) calcd for C $13 \mathrm{H}_{24} \mathrm{~N}$ $\left(\mathrm{M}^{+}+1\right)$ 194.1903. Found 194.1911.

\section{(4) N-(1-Cyclohexyl-3-phenylpropargyl)amine (3e).}

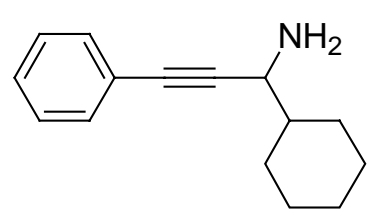

The reaction of 1e (6.402 g, $30 \mathrm{mmol})$, phthalimide (4.857 g, $33 \mathrm{mmol}), \mathrm{PPh}_{3}$ (8.653 g, $\left.33 \mathrm{mmol}\right)$, diethyl azodicarboxylate (6.270 g, 36 mmol) afforded 2e. Then the reaction of above $2 \mathbf{e}$ and $8 \mathrm{~mL}$ of $\mathrm{NH}_{2} \mathrm{NH}_{2} \cdot \mathrm{H}_{2} \mathrm{O}$ in $230 \mathrm{~mL}$ of absolute EtOH afforded $2.973 \mathrm{~g}$ (47 \%, two steps) of 3e: liquid; ${ }^{1} \mathrm{H}$ NMR (300 MHz, $\mathrm{CDCl}_{3}$ ) $\delta$ 7.48-7.37 (m, $\left.2 \mathrm{H}\right), 7.36-7.23$ (m, $\left.3 \mathrm{H}\right), 3.59$ (d, $\left.J=5.4 \mathrm{~Hz}, 1 \mathrm{H}\right), 1.98-1.62$ (m, 5 H), $1.62-1.39$ (m, 2 H), 1.39-1.06 (m, $6 \mathrm{H}) ;{ }^{13} \mathrm{C}$ NMR (75.4 MHz, $\left.\mathrm{CDCl}_{3}\right) \delta$ 131.5, 128.1, 127.8, 123.3, 83.2, 49.3, 44.3, 29.7, 28.4, 26.4, 26.1, 26.0; MS (EI) m/z (\%) 213 ( $\mathrm{M}^{+}$, 1.37), 130 (100); IR (neat) 3374, 2221, $1598 \mathrm{~cm}^{-1}$; HRMS calcd for $\mathrm{C}_{15} \mathrm{H}_{19} \mathrm{~N}\left(\mathrm{M}^{+}\right)$213.1518. Found 213.1500. 
(5) $N$-(2-Methyl-7-phenylhept-4-yn-3-yl)amine (3f).

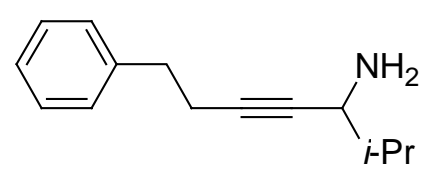

The reaction of $\mathbf{1 f}$ (4.145 g, $20.5 \mathrm{mmol}$ ), phthalimide (3.330 g, $22.65 \mathrm{mmol}), \mathrm{PPh}_{3}$ (5.909 g, 22.55 mmol), diethyl azodicarboxylate (4.310 g, $24.77 \mathrm{mmol}$ ) afforded $4.548 \mathrm{~g}(67 \%)$ of $\mathbf{2 f}$. Then the reaction of above $2 \mathbf{f}(4.548 \mathrm{~g}, 13.74 \mathrm{mmol})$ and $8 \mathrm{~mL}$ of $\mathrm{NH}_{2} \mathrm{NH}_{2} \cdot \mathrm{H}_{2} \mathrm{O}$ in $200 \mathrm{~mL}$ of absolute EtOH afforded 2.061 g (75 \%) of 3f: liquid; ${ }^{1} \mathrm{H}$ NMR (300 MHz, $\mathrm{CDCl}_{3}$ ) $\delta$ 7.35-7.15 (m, $5 \mathrm{H}$ ), 3.35 (dt, $J=2.1 \mathrm{and} 5.4 \mathrm{~Hz}, 1 \mathrm{H}$ ), 2.81 (t, $J=7.5 \mathrm{~Hz}, 2 \mathrm{H}), 2.48(\mathrm{dt}, J=2.1$ and $7.5 \mathrm{~Hz}, 2 \mathrm{H}), 1.79-1.60(\mathrm{~m}, 1 \mathrm{H}), 1.46(\mathrm{bs}, 2 \mathrm{H}), 0.93(\mathrm{~d}, J=6.9 \mathrm{~Hz}, 3 \mathrm{H}), 0.92(\mathrm{~d}, J=6.9 \mathrm{~Hz}, 3 \mathrm{H}) ;{ }^{13} \mathrm{C}$ NMR (75.4 MHz, $\left.\mathrm{CDCl}_{3}\right) \delta 140.7,128.4,128.2,126.1,82.6,82.5,49.6,35.2,34.4,20.8,19.1,17.5$; MS (EI) m/z (\%) 202 (M+1, 3.03$), 158$ (100); IR (neat) 3374, 2235, $1603 \mathrm{~cm}^{-1}$; HRMS calcd for $\mathrm{C}_{11} \mathrm{H}_{12} \mathrm{~N}\left(\mathrm{M}^{+}-\mathrm{C}_{3} \mathrm{H}_{7}\right)$ 158.0970. Found 158.0971.

\section{(6) $N$-(2,6,6-Trimethylhept-4-yn-3-yl)amine (3g).}

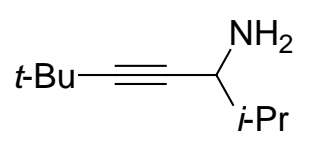

The reaction of $1 \mathrm{~g}$ (3.776 g, $24.5 \mathrm{mmol}$ ), phthalimide (3.968 g, $26.95 \mathrm{mmol}), \mathrm{PPh}_{3}$ (7.068 g, 26.95 mmol), diethyl azodicarboxylate (5.153 g, 
$29.6 \mathrm{mmol}$ ) afforded $5.359 \mathrm{~g}(67 \%)$ of $\mathbf{2 g}$. Then the reaction of above $\mathbf{2 g}(5.359 \mathrm{~g}, 18.9 \mathrm{mmol})$ and $8 \mathrm{~mL}$ of $\mathrm{NH}_{2} \mathrm{NH}_{2} \cdot \mathrm{H}_{2} \mathrm{O}$ in $200 \mathrm{~mL}$ of absolute EtOH afforded 1.183 g (41 \%) of 3g: liquid; ${ }^{1} \mathrm{H}$ NMR (300 MHz, $\left.\mathrm{CDCl}_{3}\right) \delta 3.37$ (d, $\left.J=5.7 \mathrm{~Hz}, 1 \mathrm{H}\right), 1.81-1.65$ (m, $\left.1 \mathrm{H}\right), 1.41$ (bs, $\left.2 \mathrm{H}\right), 1.21$ (s, $9 \mathrm{H}), 0.97$ (d, $J=3.6 \mathrm{~Hz}, 6 \mathrm{H}) ;{ }^{13} \mathrm{C}$ NMR $\left(75.4 \mathrm{MHz}, \mathrm{CDCl}_{3}\right) \delta$ 91.7, 80.2, 49.4, 34.6, 31.1, 27.2, 19.2, 17.4; MS (EI) m/z (\%) $152\left(\mathrm{M}^{+}-1,0.88\right)$, 110 (100); IR (neat) 3375, 2228, $1597 \mathrm{~cm}^{-1}$; HRMS (MALDI/DHB) calcd for $\mathrm{C}_{10} \mathrm{H}_{20} \mathrm{~N}\left(\mathrm{M}^{+}+1\right)$ 154.1590. Found 154.1599.

\section{The following compounds were prepared according to Typical Procedure II.}

\section{(1) $N$-(Non-4-yn-3-yl)-N-benzylamine (4b).}

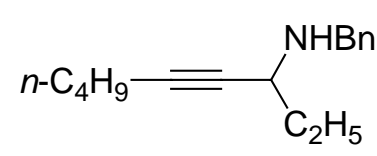

The reaction of $\mathbf{3 b}$ (692 mg, $4.98 \mathrm{mmol}$ ), $\mathrm{K}_{2} \mathrm{CO}_{3}$ (721 mg, $5.22 \mathrm{mmol}$ ), and $\mathrm{PhCH}_{2} \mathrm{Br}$ (934 mg, $5.46 \mathrm{mmol}$ ) afforded $525 \mathrm{mg}$ (46 \%) of $4 \mathbf{b}$ : liquid; ${ }^{1} \mathrm{H}$ NMR (300 MHz, $\left.\mathrm{CDCl}_{3}\right) \delta$ 7.46-7.20 (m, 5 H), 4.01 (d, $\left.J=12.9 \mathrm{~Hz}, 1 \mathrm{H}\right), 3.80$ (d, $\left.J=12.9 \mathrm{~Hz}, 1 \mathrm{H}\right), 3.35-3.24$ (m, $\left.1 \mathrm{H}\right), 2.24(\mathrm{dt}, J=$ 1.9 and $6.9 \mathrm{~Hz}, 2 \mathrm{H}), 1.79-1.35$ (m, $7 \mathrm{H}), 1.00$ (t, $J=7.5 \mathrm{~Hz}, 3 \mathrm{H}), 0.93$ (t, $J=7.2 \mathrm{~Hz}, 3 \mathrm{H}) ;{ }^{13} \mathrm{C}$ NMR $\left(75.4 \mathrm{MHz}, \mathrm{CDCl}_{3}\right) \delta 140.3,128.4,128.3$, 126.9, 84.0, 81.1, 51.4, 51.0, 31.1, 29.3, 21.9, 18.4, 13.6, 10.5; MS (EI) m/z (\%) $230\left(\mathrm{M}^{+}+1,1.36\right), 200\left(\mathrm{M}^{+}-\mathrm{C}_{2} \mathrm{H}_{5}, 73.33\right)$, 91 (100); IR (neat) 3309, 2235, $1604 \mathrm{~cm}^{-1}$; HRMS (MALDI/DHB) calcd for $\mathrm{C}_{16} \mathrm{H}_{24} \mathrm{~N}\left(\mathrm{M}^{+}+1\right)$ 230.1903. Found 230.1917. 
(2) $N$-(2-Methylnon-4-yn-3-yl)- $N$-benzylamine (4c).

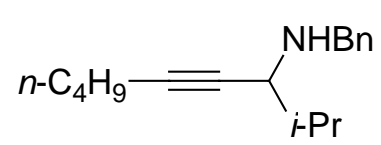

The reaction of 3c (73 mg, $0.48 \mathrm{mmol}), \mathrm{K}_{2} \mathrm{CO}_{3}$ (74 mg, $\left.0.54 \mathrm{mmol}\right)$, and $\mathrm{PhCH}_{2} \mathrm{Br}(120 \mathrm{mg}, 0.71 \mathrm{mmol})$ afforded $88 \mathrm{mg}$ (76 \%) of $4 \mathbf{c}$ : liquid; ${ }^{1} \mathrm{H}$ NMR (300 MHz, $\left.\mathrm{CDCl}_{3}\right) \delta$ 7.45-7.18 (m, $5 \mathrm{H}$ ), 4.02 (d, $\left.J=12.9 \mathrm{~Hz}, 1 \mathrm{H}\right), 3.79$ (d, $\left.J=12.9 \mathrm{~Hz}, 1 \mathrm{H}\right), 3.16$ (dt, $J=1.6 \mathrm{and} 3.7 \mathrm{~Hz}, 1$ H), 2.24 (dt, $J=2.1$ and $6.8 \mathrm{~Hz}, 2 \mathrm{H}), 1.92-1.75$ (m, $1 \mathrm{H}), 1.60-1.38$ (m, $4 \mathrm{H}), 1.31$ (bs, $1 \mathrm{H}), 0.98$ (d, $J=6.9 \mathrm{~Hz}, 6 \mathrm{H}), 0.93$ (t, $J=7.2 \mathrm{~Hz}, 3 \mathrm{H})$;

${ }^{13} \mathrm{C}$ NMR $\left(75.4 \mathrm{MHz}, \mathrm{CDCl}_{3}\right) \delta 140.4,128.3,128.3,126.8,84.5,79.7,55.7,51.6,32.8,31.1,21.9,19.7,18.4,17.8,13.6$; MS (EI) $m / z(\%) 243$ ( $\left.\mathrm{M}^{+}, 0.09\right), 242\left(\mathrm{M}^{+}-1,0.37\right), 200$ (100); IR (neat) 3325, 2240, $1605 \mathrm{~cm}^{-1}$; HRMS calcd for $\mathrm{C}_{17} \mathrm{H}_{25} \mathrm{~N}\left(\mathrm{M}^{+}\right)$243.1982. Found 243.1980.

(3) $N$-(1-Cyclohexyl-2-heptynyl)- $N$-benzylamine (4d).

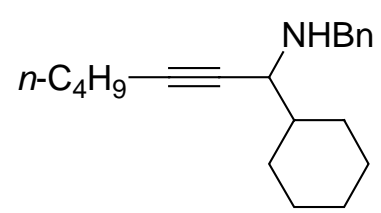

The reaction of 3d (765 mg, $3.96 \mathrm{mmol}), \mathrm{K}_{2} \mathrm{CO}_{3}$ (575 mg, $\left.4.17 \mathrm{mmol}\right)$, and $\mathrm{PhCH}_{2} \mathrm{Br}$ (763 mg, $\left.4.46 \mathrm{mmol}\right)$ afforded $715 \mathrm{mg}(64 \%)$ of $4 \mathbf{d}$ : liquid; ${ }^{1} \mathrm{H}$ NMR (300 MHz, $\left.\mathrm{CDCl}_{3}\right) \delta$ 7.48-7.15 (m, $\left.5 \mathrm{H}\right), 4.02$ (d, $\left.J=12.9 \mathrm{~Hz}, 1 \mathrm{H}\right), 3.78$ (d, $\left.J=12.9 \mathrm{~Hz}, 1 \mathrm{H}\right), 3.23-2.10(\mathrm{~m}, 1 \mathrm{H}), 2.24(\mathrm{dt}, J=$ 
1.9 and $6.9 \mathrm{~Hz}, 2 \mathrm{H}), 1.90-1.60$ (m, $5 \mathrm{H}), 1.60-1.36$ (m, $5 \mathrm{H}), 1.36-1.05(\mathrm{~m}, 6 \mathrm{H}), 0.94(\mathrm{t}, J=7.1 \mathrm{~Hz}, 3 \mathrm{H})$; ${ }^{13} \mathrm{C} \mathrm{NMR}(75.4 \mathrm{MHz}, \mathrm{CDCl} 3) \delta 140.4$, 128.3, 128.3, 126.8, 84.6, 80.2, 55.0, 51.6, 42.6, 31.2, 30.2, 28.5, 26.5, 26.2, 26.1, 21.9, 18.4, 13.6; MS (EI) m/z (\%) 284 (M+1, 2.03$), 283$ (M+', 0.94), 200 (100); IR (neat) 3370, 2228, $1604 \mathrm{~cm}^{-1}$; HRMS (MALDI/DHB) calcd for $\mathrm{C}_{20} \mathrm{H}_{30} \mathrm{~N}\left(\mathrm{M}^{+}+1\right)$ 284.2373. Found 284.2372.

\section{(4) $N$-(2-Methyl-7-phenylhept-4-yn-3-yl)- $N$-benzylamine (4f).}

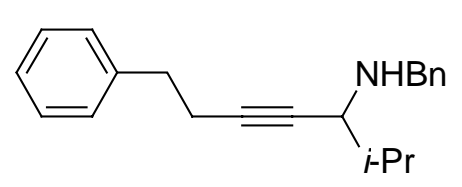

The reaction of $\mathbf{3 f}$ (593 mg, $2.95 \mathrm{mmol}$ ), $\mathrm{K}_{2} \mathrm{CO}_{3}$ (421 mg, $3.05 \mathrm{mmol}$ ), and $\mathrm{PhCH}_{2} \mathrm{Br}$ (606 mg, $3.54 \mathrm{mmol}$ ) afforded $606 \mathrm{mg}$ (71 \%) of $\mathbf{4 f}$ :

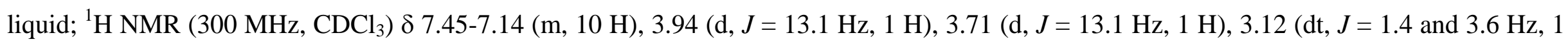
H), 2.84 (t, $J=7.5 \mathrm{~Hz}, 2 \mathrm{H}), 2.55$ (dt, $J=1.8$ and $7.5 \mathrm{~Hz}, 2 \mathrm{H}), 1.90-1.70$ (m, $1 \mathrm{H}), 1.31$ (bs, $1 \mathrm{H}), 0.94$ (d, $J=6.8 \mathrm{~Hz}, 3 \mathrm{H}), 0.93$ (d, $J=6.8 \mathrm{~Hz}$, $3 \mathrm{H}) ;{ }^{13} \mathrm{C}$ NMR $\left(75.4 \mathrm{MHz}, \mathrm{CDCl}_{3}\right) \delta 140.7,140.3,128.5,128.3,128.3,128.2,126.8,126.2,83.7,80.6,55.6,51.5,35.4,32.7,20.8,19.7,17.8$; MS (EI) m/z (\%) $292\left(\mathrm{M}^{+}+1,1.31\right), 248\left(\mathrm{M}^{+}-\mathrm{C}_{3} \mathrm{H}_{7}, 75.23\right), 91$ (100); IR (neat) 3324, 2235, $1604 \mathrm{~cm}^{-1}$; HRMS (MALDI/DHB) calcd for C $21 \mathrm{H}_{26} \mathrm{~N}$ $\left(\mathrm{M}^{+}+1\right)$ 292.2060. Found 292.2044. 


\section{The following compounds were prepared according to Typical Procedure III.}

\section{(1) (E)- $\alpha-(1-C h l o r o p e n t y l i d e n e)-\beta$-ethyl- $\beta$-lactam ((E)-6b).}

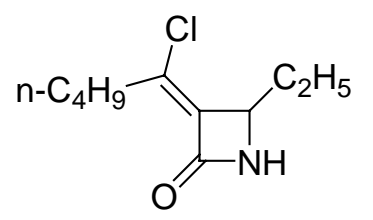

The reaction of 3b (139 mg, $1.0 \mathrm{mmol}), \mathrm{PdCl}_{2}$ (9 mg, $\left.0.05 \mathrm{mmol}\right), \mathrm{CuCl}_{2}$ (268 mg, $\left.1.99 \mathrm{mmol}\right)$, and benzoquinone (112 mg, $\left.1.04 \mathrm{mmol}\right)$ was stirred at $45-50{ }^{\circ} \mathrm{C}$ for $8 \mathrm{~h}$ to afford $96 \mathrm{mg}$ (48 \%) of (E)-6b: liquid; ${ }^{1} \mathrm{H}$ NMR (300 MHz, $\left.\mathrm{CDCl}_{3}\right) \delta 6.90$ (bs, $1 \mathrm{H}$ ), 4.18 (dd, $J=3.2 \mathrm{and} 7.4 \mathrm{~Hz}, 1$ H), 2.85-2.65 (m, 2 H), 2.08-1.90 (m, 1 H), 1.77-1.52 (m, 3 H), 1.45-1.29 (m, 2 H), 0.96 (t, $J=7.4$ Hz, 3 H), 0.93 (t, $J=7.5$ Hz, 3 H); ${ }^{13} \mathrm{C} \mathrm{NMR}$ $\left(75.4 \mathrm{MHz}, \mathrm{CDCl}_{3}\right) \delta 162.7,137.2,136.7,58.1,34.5,29.1,24.6,21.5,13.7,8.9 ; \mathrm{MS}(\mathrm{EI}) \mathrm{m} / \mathrm{z}(\%) 203\left(\mathrm{M}^{+}\left({ }^{37} \mathrm{Cl}\right), 2.61\right), 201$ (M $\left.\left.{ }^{+}{ }^{35} \mathrm{Cl}\right), 7.87\right), 81$ (100); IR (neat) 3249, 1750, $1707 \mathrm{~cm}^{-1}$; HRMS (MALDI/DHB) calcd for $\mathrm{C}_{10} \mathrm{H}_{17}{ }^{35} \mathrm{ClNO}\left(\mathrm{M}^{+}+1\right)$ 202.0993. Found 202.1008. 


\section{(2) (E)- $\alpha-(1-C h l o r o p e n t y l i d e n e)-\beta-(i-p r o p y l)-\beta-\operatorname{lactam}((E)-6 c)$ :}

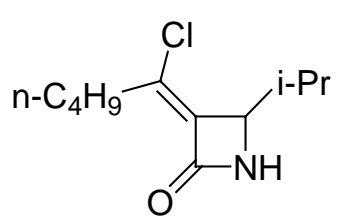

The reaction of 3c (148 mg, $0.97 \mathrm{mmol}), \mathrm{PdCl}_{2}$ (9 mg, $\left.0.051 \mathrm{mmol}\right), \mathrm{CuCl}_{2}$ (267 mg, $1.98 \mathrm{mmol}$ ), and benzoquinone (110 mg, $1.02 \mathrm{mmol}$ ) was stirred at $45-50{ }^{\circ} \mathrm{C}$ for $8 \mathrm{~h}$ to afford $110 \mathrm{mg}$ (53 \%) of (E)-6c: liquid; ${ }^{1} \mathrm{H}$ NMR (300 MHz, $\left.\mathrm{CDCl}_{3}\right) \delta 6.88$ (bs, $\left.1 \mathrm{H}\right), 4.15$ (d, $J=2.7 \mathrm{~Hz}, 1 \mathrm{H}$ ), 2.95-2.61 (m, 2 H), 2.36-2.12 (m, 1 H), 1.70-1.51 (m, 2 H), 1.50-1.28 (m, 2 H), 1.00 (d, $J=6.9$ Hz, 3 H), 0.94 (d, $J=7.2$ Hz, 3 H), 0.90 (d, $J=$ 6.6 Hz, $3 \mathrm{H}) ;{ }^{13} \mathrm{C}$ NMR $\left(75.4 \mathrm{MHz}, \mathrm{CDCl}_{3}\right) \delta 163.0,136.7,136.6,62.4,34.5,29.1,28.4,21.5,19.3,15.4,13.7$; MS (EI) $\mathrm{m} / \mathrm{z}(\%) 217\left(\mathrm{M}^{+}\left({ }^{37} \mathrm{Cl}\right)\right.$, 1.35), $215\left(\mathrm{M}^{+}\left({ }^{35} \mathrm{Cl}\right), 3.92\right), 172$ (100); IR (neat) 3238, 1748, $1713 \mathrm{~cm}^{-1}$; HRMS (MALDI/DHB) calcd for $\mathrm{C}_{11} \mathrm{H}_{19}{ }^{35} \mathrm{ClNO}\left(\mathrm{M}^{+}+1\right) 216.1150$. Found 216.1161.

(3) (E)- $\alpha$-(1-Chloropentylidene)- $\beta$-cyclohexyl- $\beta$-lactam ((E)-6d): 


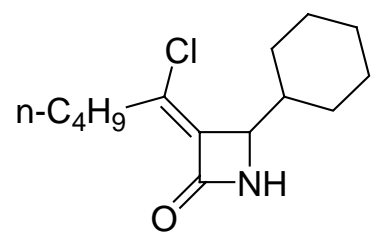

The reaction of 3d (151 mg, $0.78 \mathrm{mmol}), \mathrm{PdCl}_{2}$ (7 mg, $0.04 \mathrm{mmol}$ ), $\mathrm{CuCl}_{2}$ (215 mg, $1.59 \mathrm{mmol}$ ), and benzoquinone (89 mg, $0.82 \mathrm{mmol}$ ) was stirred at $45-50{ }^{\circ} \mathrm{C}$ for $8 \mathrm{~h}$ to afford $100 \mathrm{mg}$ (50 \%) of (E)-6d: solid, mp 100.5-102 ${ }^{\circ} \mathrm{C}\left(n\right.$-Hexane/Ethyl acetate); ${ }^{1} \mathrm{H} \mathrm{NMR} \mathrm{(300} \mathrm{MHz,} \mathrm{CDCl} 3$ ) $\delta 5.93$ (bs, $1 \mathrm{H}), 4.12$ (d, $J=3 \mathrm{~Hz}, 1 \mathrm{H}), 2.90-2.65$ (m, $2 \mathrm{H}), 1.98-1.52$ (m, $9 \mathrm{H}), 1.48-0.98$ (m, $6 \mathrm{H}), 0.93$ (t, $J=7.4 \mathrm{~Hz}, 3 \mathrm{H}) ;{ }^{13} \mathrm{C}$ NMR $(75.4$ $\left.\mathrm{MHz}, \mathrm{CDCl}_{3}\right) \delta 163.0,136.5,136.3,62.0,38.2,34.5,29.9,29.2,26.14,26.09,25.9,25.7,21.5,13.7 ; \mathrm{MS}(\mathrm{EI}) \mathrm{m} / \mathrm{z}(\%) 257\left(\mathrm{M}^{+}\left({ }^{37} \mathrm{Cl}\right), 2.43\right), 255$ (M ${ }^{+}\left({ }^{35} \mathrm{Cl}\right)$, 6.48), 172 (100); IR (KBr) 3205, 1741, $1703 \mathrm{~cm}^{-1}$; Anal. Calcd for $\mathrm{C}_{14} \mathrm{H}_{22} \mathrm{ClNO}$ : C, 65.74; H, 8.67; N, 5.48. Found: C, 65.55; H, 8.38; N, 5.48. 


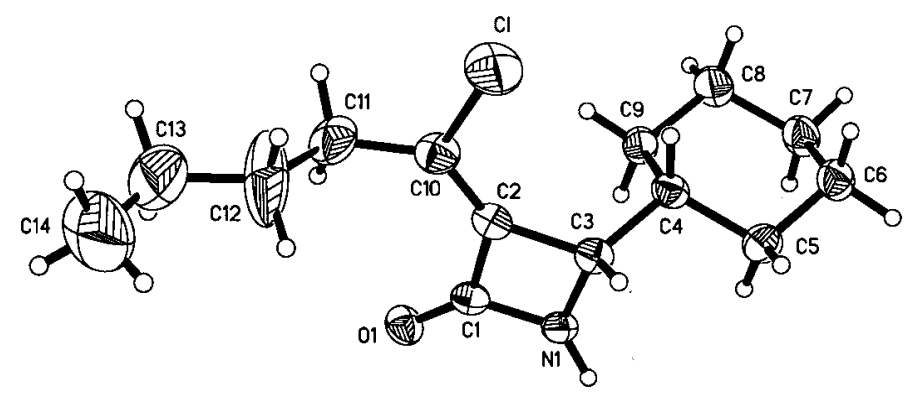

Fig. 1. The X-ray structure of compound (E)-6d.

(4) (E)- $\alpha-(1-C h l o r o b e n z y l i d e n e)-\beta-c y c l o h e x y l-\beta-\operatorname{lactam}((E)-6 e)$.

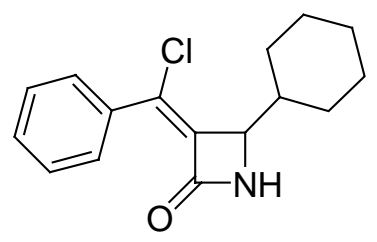

The reaction of $3 \mathbf{e}(167 \mathrm{mg}, 0.78 \mathrm{mmol}), \mathrm{PdCl}_{2}$ (7 mg, $\left.0.04 \mathrm{mmol}\right), \mathrm{CuCl}_{2}$ (216 mg, $\left.1.60 \mathrm{mmol}\right)$, and benzoquinone (89 mg, $\left.0.82 \mathrm{mmol}\right)$ was stirred at $45-50{ }^{\circ} \mathrm{C}$ for $10.5 \mathrm{~h}$ to afford $69 \mathrm{mg}(32 \%)$ of $(\boldsymbol{E})$-6e: solid, mp 123-124 ${ }^{\circ} \mathrm{C}$ ( $n$-Hexane/Ethyl acetate); ${ }^{1} \mathrm{H}$ NMR $(300 \mathrm{MHz}, \mathrm{CDCl})_{3} \delta$ 8.05-7.90 (m, 2 H), 7.50-7.35 (m, 3 H), 6.92 (bs, 1 H), 4.22 (d, J = 3 Hz, 1 H), 2.22-1.95 (m, 1 H), 1.90-1.55 (m, 5 H), $1.45-0.95$ (m, 5 H); ${ }^{13} \mathrm{C}$ 
NMR (75.4 MHz, $\left.\mathrm{CDCl}_{3}\right) \delta 161.3,137.5,133.6,132.6,130.3,128.4,128.1,61.8,37.8,29.9,26.3,26.1,25.7,25.4$; MS (EI) m/z (\%) 277 ( $\mathrm{M}^{+}\left({ }^{37} \mathrm{Cl}\right)$, 3.40), 275 ( $\mathrm{M}^{+}\left({ }^{35} \mathrm{Cl}\right)$, 10.57), 192 (100); IR (KBr) 3198, 1736, $1657 \mathrm{~cm}^{-1}$; Anal. Calcd for $\mathrm{C}_{16} \mathrm{H}_{18} \mathrm{ClNO:} \mathrm{C,} \mathrm{69.68;} \mathrm{H,} \mathrm{6.58;} \mathrm{N,} \mathrm{5.08.}$

Found: C, 69.58; H, 6.57; N, 5.10.

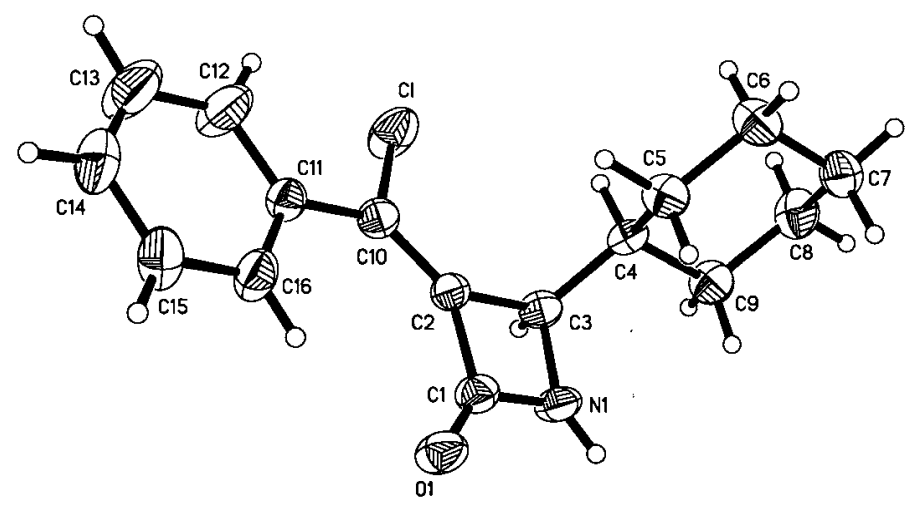

Fig. 2. The X-ray structure of compound (E)-6e.

(5) (E)- $\alpha-(1-C h l o r o-3-p h e n y l p r o p y l i d e n e)-\beta-(i-p r o p y l)-\beta-\operatorname{lactam}((E)-6 f)$. 


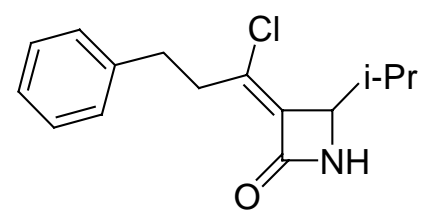

The reaction of $3 \mathbf{f}$ (156 mg, $0.78 \mathrm{mmol}), \mathrm{PdCl}_{2}$ (7 mg, $\left.0.04 \mathrm{mmol}\right), \mathrm{CuCl}_{2}$ (220 mg, $1.63 \mathrm{mmol}$ ), and benzoquinone (90 mg, $\left.0.83 \mathrm{mmol}\right)$ was stirred at $45-50{ }^{\circ} \mathrm{C}$ for $8 \mathrm{~h}$ to afford $86 \mathrm{mg}$ (42 \%) of (E)-6f: liquid; ${ }^{1} \mathrm{H}$ NMR (300 MHz, $\left.\mathrm{CDCl}_{3}\right) \delta$ 7.38-7.15 (m, $5 \mathrm{H}$ ), 6.06 (bs, $\left.1 \mathrm{H}\right), 4.09$ (d, $J=$ 3.6 Hz, $1 \mathrm{H}), 3.35-3.16$ (m, $1 \mathrm{H}), 3.10-2.85$ (m, $3 \mathrm{H}), 2.30-2.10(\mathrm{~m}, 1 \mathrm{H}), 0.92(\mathrm{~d}, J=6.8 \mathrm{~Hz}, 3 \mathrm{H}), 0.70(\mathrm{~d}, J=6.8 \mathrm{~Hz}, 3 \mathrm{H})$; ${ }^{13} \mathrm{C} \mathrm{NMR}(75.4$ $\left.\mathrm{MHz}, \mathrm{CDCl}_{3}\right) \delta 162.6,139.5,137.4,135.0,128.5,128.3,126.1,62.4,36.0,33.2,28.3,19.2,15.1 ; \mathrm{MS}(\mathrm{EI}) \mathrm{m} / \mathrm{z}(\%) 265\left(\mathrm{M}^{+}\left({ }^{37} \mathrm{Cl}\right), 4.58\right), 263$ ( $\left.\mathrm{M}^{+}\left({ }^{35} \mathrm{Cl}\right), 13.83\right), 91(100)$; IR (neat) 3243, 1746, $1709 \mathrm{~cm}^{-1}$; HRMS calcd for $\mathrm{C}_{15} \mathrm{H}_{18}{ }^{35} \mathrm{ClNO}\left(\mathrm{M}^{+}\right)$263.1077. Found 263.1067.

\section{The following compounds were prepared according to Typical Procedure IV.}

\section{(1) (E)- $\alpha$-(1-Chloropentylidene)- $\beta$-(n-pentyl)- $N$-benzyl- $\beta$-lactam ((E)-6g).}

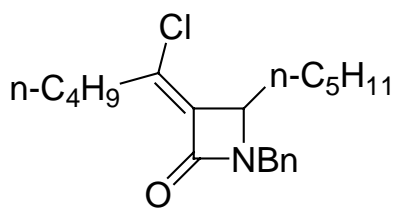

The reaction of 4a (143 mg, $0.53 \mathrm{mmol}), \mathrm{PdCl}_{2}$ (5 mg, $0.028 \mathrm{mmol}$ ), $\mathrm{CuCl}_{2}$ (148 mg, $1.10 \mathrm{mmol}$ ), and benzoquinone (62 mg, $0.57 \mathrm{mmol}$ ) 
was stirred for $12 \mathrm{~h}$ at $45-50{ }^{\circ} \mathrm{C}$ to afford $108 \mathrm{mg}(61 \%)$ of $(\boldsymbol{E})$-6g: liquid; ${ }^{1} \mathrm{H}$ NMR (300 MHz, $\left.\mathrm{CDCl}_{3}\right) \delta$ 7.41-7.20 (m, $\left.5 \mathrm{H}\right)$, $4.74(\mathrm{~d}, J=15.2$ Hz, 1 H), 4.15 (d, $J=15.2$ Hz, 1 H), 4.07 (t, $J=4.2$ Hz, 1 H), 2.91-2.65 (m, 2 H), 1.83-1.50 (m, 4 H), 1.48-1.06 (m, 8 H), 0.94 (t, $J=7.4$ Hz, 3 H), 0.84 (t, $J=6.9 \mathrm{~Hz}, 3 \mathrm{H}) ;{ }^{13} \mathrm{C}$ NMR $\left(75.4 \mathrm{MHz}, \mathrm{CDCl}_{3}\right) \delta 161.8,136.2,135.7,135.1,128.7,128.2,127.7,59.7,44.7,34.8,31.6,29.1,23.5$, 22.4, 21.5, 13.9, 13.7; MS (EI) $\mathrm{m} / \mathrm{z}$ (\%) $334\left(\mathrm{M}^{+}-1\left({ }^{37} \mathrm{Cl}\right), 0.97\right), 332\left(\mathrm{M}^{+}-1\left({ }^{35} \mathrm{Cl}\right), 1.87\right), 91$ (100); IR (neat) $1747,1707 \mathrm{~cm}{ }^{-1}$; HRMS (MALDI/DHB) calcd for $\mathrm{C}_{20} \mathrm{H}_{29}{ }^{35} \mathrm{ClNO}\left(\mathrm{M}^{+}+1\right)$ 334.1932. Found 334.1953.

\section{(2) (E)- $\alpha$-(1-Chloropentylidene)- $\beta$-ethyl- $N$-benzyl- $\beta$-lactam ((E)-6h).}

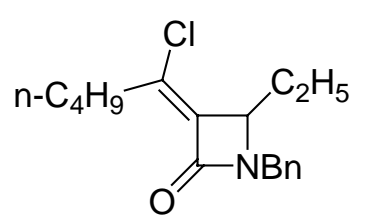

The reaction of $\mathbf{4 b}$ (135 mg, $0.59 \mathrm{mmol}), \mathrm{PdCl}_{2}$ (6 mg, $\left.0.034 \mathrm{mmol}\right), \mathrm{CuCl}_{2}$ (164 mg, $1.21 \mathrm{mmol}$ ), and benzoquinone (66 mg, $\left.0.61 \mathrm{mmol}\right)$ was stirred for $12 \mathrm{~h}$ at $45-50{ }^{\circ} \mathrm{C}$ to afford $134 \mathrm{mg}$ (78 \%) of (E)-6h: liquid; ${ }^{1} \mathrm{H}$ NMR (300 MHz, $\left.\mathrm{CDCl}_{3}\right) \delta$ 7.41-7.20 (m, $\left.5 \mathrm{H}\right), 4.77$ (d, $J=15.2$ Hz, $1 \mathrm{H}), 4.11$ (d, $J=15.2 \mathrm{~Hz}, 1 \mathrm{H}), 4.07$ (d, $J$ = $3.9 \mathrm{~Hz}, 1 \mathrm{H}), 2.93-2.65$ (m, $2 \mathrm{H}), 1.93-1.68$ (m, $2 \mathrm{H}), 1.68-1.50$ (m, 2 H), $1.46-1.28$ (m, 2 H), 0.94 (t, $J=7.2 \mathrm{~Hz}, 3 \mathrm{H}), 0.83$ (t, $J=7.5 \mathrm{~Hz}, 3 \mathrm{H}) ;{ }^{13} \mathrm{C} \mathrm{NMR}\left(75.4 \mathrm{MHz}, \mathrm{CDCl}_{3}\right) \delta$ 161.8, 135.7, 135.6, 135.3, 128.7, 128.2, 127.7, 60.3, 44.5, 
34.8, 29.2, 21.6, 21.5, 13.7, 7.8; MS (EI) m/z (\%) 293 ( $\left.\mathrm{M}^{+}\left({ }^{37} \mathrm{Cl}\right), 3.12\right), 291\left(\mathrm{M}^{+}\left({ }^{35} \mathrm{Cl}\right), 9.77\right), 91$ (100); IR (neat) $1748,1717 \mathrm{~cm}{ }^{-1}$; HRMS (MALDI/DHB) calcd for $\mathrm{C}_{17} \mathrm{H}_{23}{ }^{35} \mathrm{ClNO}\left(\mathrm{M}^{+}+1\right)$ 292.1463. Found 292.1488.

\section{(3) (E)- $\alpha$-(1-Chloropentylidene)- $\beta$-cyclohexyl- $N$-benzyl- $\beta$-lactam ((E)-6j).}

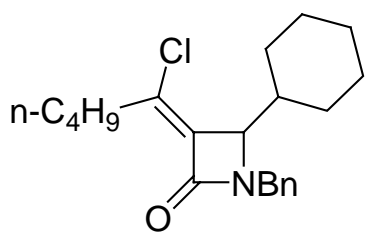

The reaction of 4d (141 mg, $0.5 \mathrm{mmol}), \mathrm{PdCl}_{2}$ (5 mg, $\left.0.028 \mathrm{mmol}\right), \mathrm{CuCl}_{2}$ (140 mg, $1.04 \mathrm{mmol}$ ), and benzoquinone (58 mg, $0.54 \mathrm{mmol}$ ) was stirred for $12 \mathrm{~h}$ at $45-50{ }^{\circ} \mathrm{C}$ to afford $108 \mathrm{mg}(63 \%)$ of $(\boldsymbol{E})-\mathbf{6 j}$ : liquid; ${ }^{1} \mathrm{H}$ NMR (300 MHz, $\left.\mathrm{CDCl}_{3}\right) \delta$ 7.43-7.18 (m, $\left.5 \mathrm{H}\right), 4.84(\mathrm{~d}, \mathrm{~J}=15.3$ Hz, $1 \mathrm{H}), 4.11$ (d, $J=15.3 \mathrm{~Hz}, 1 \mathrm{H}), 3.95$ (d, $J=1.5 \mathrm{~Hz}, 1 \mathrm{H})$, 2.95-2.80 (m, $1 \mathrm{H}), 2.80-2.65$ (m, $1 \mathrm{H}), 1.92-1.50$ (m, 8 H), $1.49-1.32$ (m, 2 H), 1.32-1.00 (m, $5 \mathrm{H}), 0.94$ (t, $J=7.2 \mathrm{~Hz}, 3 \mathrm{H}) ;{ }^{13} \mathrm{C}$ NMR (75.4 MHz, $\left.\mathrm{CDCl}_{3}\right) \delta$ 162.6, 135.8, 135.3, 135.2, 128.7, 128.2, 127.6, 64.7, 45.6, 38.1, 34.9, 30.0, 29.2, 27.3, 26.5, 26.2, 26.1, 21.5, 13.7; MS (EI) m/z (\%) $347\left(\mathrm{M}^{+}\left({ }^{37} \mathrm{Cl}\right), 0.74\right), 345$ ( $\left.\mathrm{M}^{+}\left({ }^{35} \mathrm{Cl}\right), 1.84\right), 91$ (100); IR (neat) 1748, 1713 $\mathrm{cm}^{-1}$; HRMS (MALDI/DHB) calcd for $\mathrm{C}_{21} \mathrm{H}_{29}{ }^{35} \mathrm{CINO}\left(\mathrm{M}^{+}+1\right)$ 346.1932. Found 346.1970. 
(4) (E)- $\alpha$-(1-Chloro-3-phenylpropylidene)- $\beta$-(i-propyl)- $N$-benzyl- $\beta$-lactam ((E)-6k).

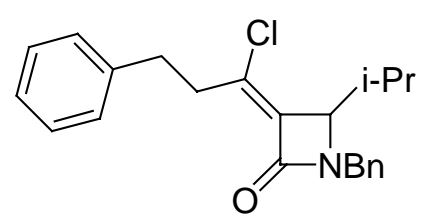

The reaction of $\mathbf{4 f}(147 \mathrm{mg}, 0.51 \mathrm{mmol}), \mathrm{PdCl}_{2}$ (5 mg, $\left.0.028 \mathrm{mmol}\right), \mathrm{CuCl}_{2}$ (139 mg, $1.03 \mathrm{mmol}$ ), and benzoquinone (56 mg, $\left.0.52 \mathrm{mmol}\right)$ was stirred for $12 \mathrm{~h}$ at $45-50{ }^{\circ} \mathrm{C}$ to afford $125 \mathrm{mg}(70 \%)$ of $(\boldsymbol{E})$-6k: liquid; ${ }^{1} \mathrm{H}$ NMR (300 MHz, $\left.\mathrm{CDCl}_{3}\right) \delta$ 7.40-7.10 (m, $\left.10 \mathrm{H}\right), 4.83(\mathrm{~d}, \mathrm{~J}=15.5$ Hz, 1 H), 3.99 (d, $J=15.5$ Hz, 1 H), 3.89 (d, $J=2.1$ Hz, 1 H), 3.31-3.18 (m, 1 H), 3.11-2.88 (m, 3 H), 2.20-2.00 (m, 1 H), 0.82 (d, $J$ = 7.5 Hz, 3 H), 0.77 (d, $J=7.2 \mathrm{~Hz}, 3 \mathrm{H}) ;{ }^{13} \mathrm{C}$ NMR $\left(75.4 \mathrm{MHz}, \mathrm{CDCl}_{3}\right) \delta$ 162.3, 139.5, 136.4, 135.5, 133.7, 128.7, 128.6, 128.3, 128.1, 127.6, 126.1, 64.9, 45.5, 36.3, 33.2, 28.0, 19.1, 16.4; MS (EI) m/z (\%) 355 ( $\left.\mathrm{M}^{+}\left({ }^{37} \mathrm{Cl}\right), 1.51\right), 353$ ( $\left.\mathrm{M}^{+}\left({ }^{35} \mathrm{Cl}\right), 4.33\right), 91$ (100); IR (neat) 1747, 1716 cm ${ }^{-1}$; HRMS (MALDI/DHB) calcd for $\mathrm{C}_{22} \mathrm{H}_{25}{ }^{35} \mathrm{ClNO}\left(\mathrm{M}^{+}+1\right)$ 354.1619. Found 354.1624.

(5) (E)- $\alpha$-(1-Chloropentylidene)- $\beta$-methyl- $N$-benzyl- $\beta$-lactam ((E)-6l).

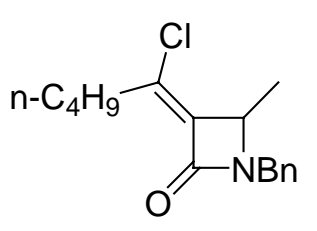


The reaction of $\mathbf{4 h}$ (125 mg, $0.58 \mathrm{mmol}), \mathrm{PdCl}_{2}$ (6 mg, $\left.0.034 \mathrm{mmol}\right), \mathrm{CuCl}_{2}$ (157 mg, $1.16 \mathrm{mmol}$ ), and benzoquinone (63 mg, $\left.0.58 \mathrm{mmol}\right)$ was stirred for $8 \mathrm{~h}$ at $30-40{ }^{\circ} \mathrm{C}$ to afford $98 \mathrm{mg}(61 \%)$ of $(\boldsymbol{E})-6 \mathbf{l}$ : liquid; ${ }^{1} \mathrm{H}$ NMR (300 MHz, $\left.\mathrm{CDCl}_{3}\right) \delta$ 7.41-7.20 (m, $\left.5 \mathrm{H}\right), 4.70$ (d, $J=15.3 \mathrm{~Hz}$, $1 \mathrm{H}), 4.18(\mathrm{~d}, J=15.3 \mathrm{~Hz}, 1 \mathrm{H}), 4.03(\mathrm{q}, J=6.1 \mathrm{~Hz}, 1 \mathrm{H}), 2.75(\mathrm{dt}, J=0.9$ and $7.4 \mathrm{~Hz}, 2 \mathrm{H}), 1.68-1.52(\mathrm{~m}, 2 \mathrm{H}), 1.48-1.22$ (m, 2 H), $1.32(\mathrm{~d}, J=$ 6.1 Hz, $3 \mathrm{H}), 0.94$ (t, $J=7.4 \mathrm{~Hz}, 3 \mathrm{H}) ;{ }^{13} \mathrm{C} \mathrm{NMR}\left(75.4 \mathrm{MHz}, \mathrm{CDCl}_{3}\right) \delta$ 161.2, 138.1, 135.7, 134.9, 128.8, 128.1, 127.7, 55.5, 43.9, 34.8, 29.2, 21.5, 15.9, 13.7; MS (EI) m/z (\%) $279\left(\mathrm{M}^{+}\left({ }^{37} \mathrm{Cl}\right), 4.46\right), 277\left(\mathrm{M}^{+}\left({ }^{35} \mathrm{Cl}\right), 13.41\right), 91$ (100); IR (neat) 1748, $1716 \mathrm{~cm}^{-1}$; HRMS calcd for $\mathrm{C}_{16} \mathrm{H}_{20}{ }^{35} \mathrm{ClNO}\left(\mathrm{M}^{+}\right)$277.1233. Found 277.1253.

\section{(6) (E)- $\alpha$-(1-Chloropentylidene)- $\beta$-phenyl- $N$-Tosyl- $\beta$-lactam ((E)-6m):}

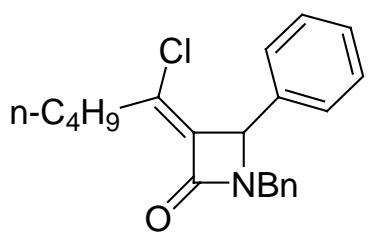

The reaction of $4 \mathbf{l}(167 \mathrm{mg}, 0.49 \mathrm{mmol}), \mathrm{PdCl}_{2}$ (5 mg, $\left.0.028 \mathrm{mmol}\right), \mathrm{CuCl}_{2}$ (137 mg, $\left.1.01 \mathrm{mmol}\right)$, and benzoquinone (59 mg, $\left.0.55 \mathrm{mmol}\right)$ was stirred for $12 \mathrm{~h}$ at $45-50{ }^{\circ} \mathrm{C}$ to afford $72 \mathrm{mg}$ (36 \%) of (E)-6m: solid, mp 103-104 ${ }^{\circ} \mathrm{C}\left(\right.$ - $-\mathrm{Hexane} / \mathrm{Ethyl}$ acetate); ${ }^{1} \mathrm{H} \mathrm{NMR}(300 \mathrm{MHz}, \mathrm{CDCl} 3$ ) $\delta$ 7.64-7.14 (m, 9 H), 5.49 (s, 1 H), 2.78 (t, $J=7.5$ Hz, 2 H), 2.39 (s, 3 H), 1.75-1.48 (m, 2 H), 1.42-1.20 (m, 2 H), 0.90 (t, $J=7.2$ Hz, 3 H); ${ }^{13} \mathrm{C}$ 
NMR (75.4 MHz, $\left.\mathrm{CDCl}_{3}\right) \delta 157.5,145.7,144.8,136.0,134.2,133.4,129.6,129.2,128.6,128.0,127.1,65.2,35.6,29.2,21.6,21.5,13.6$; MS (EI) $\mathrm{m} / \mathrm{z}$ (\%) 405 ( $\left.\mathrm{M}^{+}\left({ }^{37} \mathrm{Cl}\right), 0.66\right), 403\left(\mathrm{M}^{+}\left({ }^{35} \mathrm{Cl}\right), 1.53\right), 91$ (100); IR (KBr) 1775, $1701 \mathrm{~cm}^{-1}$; Anal. Calcd for $\mathrm{C}_{21} \mathrm{H}_{22} \mathrm{ClNO}_{3} \mathrm{~S}$ : C, 62.44; $\mathrm{H}, 5.49$; N, 3.47. Found: C, 62.37; H, 5.43; N, 3.54.

(7) $(R)-(+)-(E)-\alpha-(1-C h l o r o-3-p h e n y l p r o p y l i d e n e)-\beta-(i-p r o p y l)-N$-benzyl- $\beta-l a c t a m((R)-(+)-(E)-6 \mathbf{k})$.

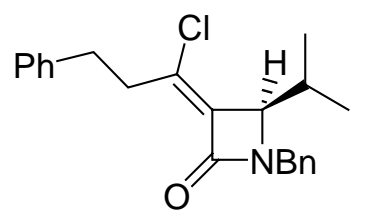

The reaction of $(\boldsymbol{R})-(+)-4 f\left(144 \mathrm{mg}, 0.49 \mathrm{mmol}, \quad 98 \%\right.$ ee), $\mathrm{PdCl}_{2}$ (6 mg, $\left.0.034 \mathrm{mmol}\right), \mathrm{CuCl}_{2}$ (140 mg, 1.04 mmol), and benzoquinone (59 mg, $0.55 \mathrm{mmol})$, afforded $86 \mathrm{mg}$ (50\% yield) of $(\boldsymbol{R})-(+)-(\boldsymbol{E})-\mathbf{6 k}$ with $98 \%$ ee as determined by HPLC analysis (Chiralcel As-H, $n$-Hexane : $i-\mathrm{PrOH}=100: 0.5,230 \mathrm{~nm}), \mathrm{t}_{\mathrm{r}} 11.2$ (major), 12.4 (minor); $[\alpha]_{\mathrm{D}}^{20}=+75.1\left(\mathrm{c}=1.0, \mathrm{CHCl}_{3}\right)$.

(8) $(R)-(+)-(E)-\alpha-(1-C h l o r o p e n t y l i d e n e)-\beta-m e t h y l-N$-benzyl- $\beta$-lactam $((R)-(+)-(E)-6 l)$. 


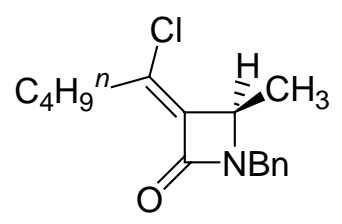

The reaction of $(\boldsymbol{R})-(+)-4 h\left(131 \mathrm{mg}, 0.61 \mathrm{mmol}, \quad 97 \%\right.$ ee), $\mathrm{PdCl}_{2}(7 \mathrm{mg}, 0.04 \mathrm{mmol}), \mathrm{CuCl}_{2}$ (175 mg, $\left.1.30 \mathrm{mmol}\right)$, and benzoquinone (74 mg, $0.69 \mathrm{mmol}$ ), afforded $93 \mathrm{mg}$ (55\% yield) of $(\boldsymbol{R})-(+)-(\boldsymbol{E})-\mathbf{6 l}$ with 94\% ee as determined by HPLC analysis (Chiralcel AD-H, $n$-Hexane : $i-\mathrm{PrOH}=90: 10,230 \mathrm{~nm}), \mathrm{t}_{\mathrm{r}} 6.4$ (major), 7.0 (minor); $[\alpha]^{20}{ }_{\mathrm{D}}=+51.7\left(\mathrm{c}=1.1, \mathrm{CHCl}_{3}\right)$.

(9) (S)-(-)-(E)- $\alpha-(1-C h l o r o p e n t y l i d e n e)-\beta-m e t h y l-N$-benzyl- $\beta$-lactam ((S)-(-)-(E)-6l).

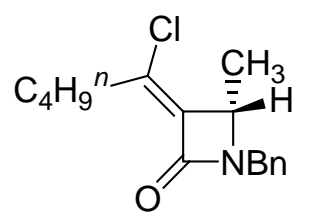

The reaction of (S)-(-)-4h (130 mg, $0.6 \mathrm{mmol}, \quad 95 \%$ ee), $\mathrm{PdCl}_{2}$ (7 mg, $\left.0.04 \mathrm{mmol}\right), \mathrm{CuCl}_{2}$ (177 mg, $\left.1.31 \mathrm{mmol}\right)$, and benzoquinone (72 mg, $0.67 \mathrm{mmol})$, afforded $89 \mathrm{mg}$ (53\% yield) of $(\boldsymbol{S})-(-)-(\boldsymbol{E})-\mathbf{6 l}$ with 93\% ee as determined by HPLC analysis (Chiralcel AD-H, $n$-Hexane $: i-\mathrm{PrOH}=$ $90: 10,230 \mathrm{~nm}), \mathrm{t}_{\mathrm{r}} 8.1$ (major), 9.1 (minor); $[\alpha]^{20}=-53.1\left(\mathrm{c}=0.97, \mathrm{CHCl}_{3}\right)$. 
(10) $(R)-(+)-(E)-\alpha-(1-C h l o r o p e n t y l i d e n e)-\beta-e t h y l-N$-benzyl- $\beta$-lactam $((R)-(+)-(E)-6 h)$.

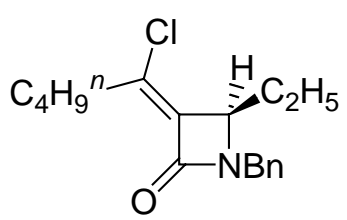

The reaction of $(\boldsymbol{R})-(+)-4 \mathbf{b}$ (134 mg, $0.59 \mathrm{mmol}, 98 \%$ ee), $\mathrm{PdCl}_{2}$ (6 mg, $\left.0.034 \mathrm{mmol}\right), \mathrm{CuCl}_{2}$ (178 mg, $1.32 \mathrm{mmol}$ ), and benzoquinone (75 mg, 0.69 mmol), afforded $84 \mathrm{mg}$ (49 yield) of $(\boldsymbol{R})-(+)-(\boldsymbol{E})-\mathbf{6 h}$ with 98\% ee as determined by HPLC analysis (Chiralcel AD-H, $n$-Hexane : $i-\mathrm{PrOH}=90: 10,230 \mathrm{~nm}), \mathrm{t}_{\mathrm{r}} 3.3$ (major), 3.6 (minor); $[\alpha]^{20}{ }_{\mathrm{D}}=+73.7\left(\mathrm{c}=1.05, \mathrm{CHCl}_{3}\right)$.

\section{(11) (S)-(-)-(E)- $\alpha-(1-C h l o r o p e n t y l i d e n e)-\beta$-ethyl- $N$-benzyl- $\beta$-lactam ((S)-(-)-(E)-6h):}

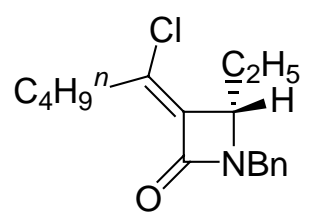

The reaction of (S)-(-)-4b (136 mg, 0.59 mmol, 96 \% ee), $\mathrm{PdCl}_{2}$ (6 mg, $\left.0.034 \mathrm{mmol}\right), \mathrm{CuCl}_{2}$ (184 mg, 1.36 mmol), and benzoquinone (72 mg, $0.67 \mathrm{mmol}$ ), afforded $98 \mathrm{mg}$ (57 yield) of (S)-(-)-(E)-6h with 91\% ee as determined by HPLC analysis (Chiralcel AD-H, $n$-Hexane : $i$-PrOH $=90: 10,230 \mathrm{~nm}), \mathrm{t}_{\mathrm{r}} 3.3$ (minor), 3.6 (major); $[\alpha]_{\mathrm{D}}^{20}=-56.1\left(\mathrm{c}=1.015, \mathrm{CHCl}_{3}\right)$. 

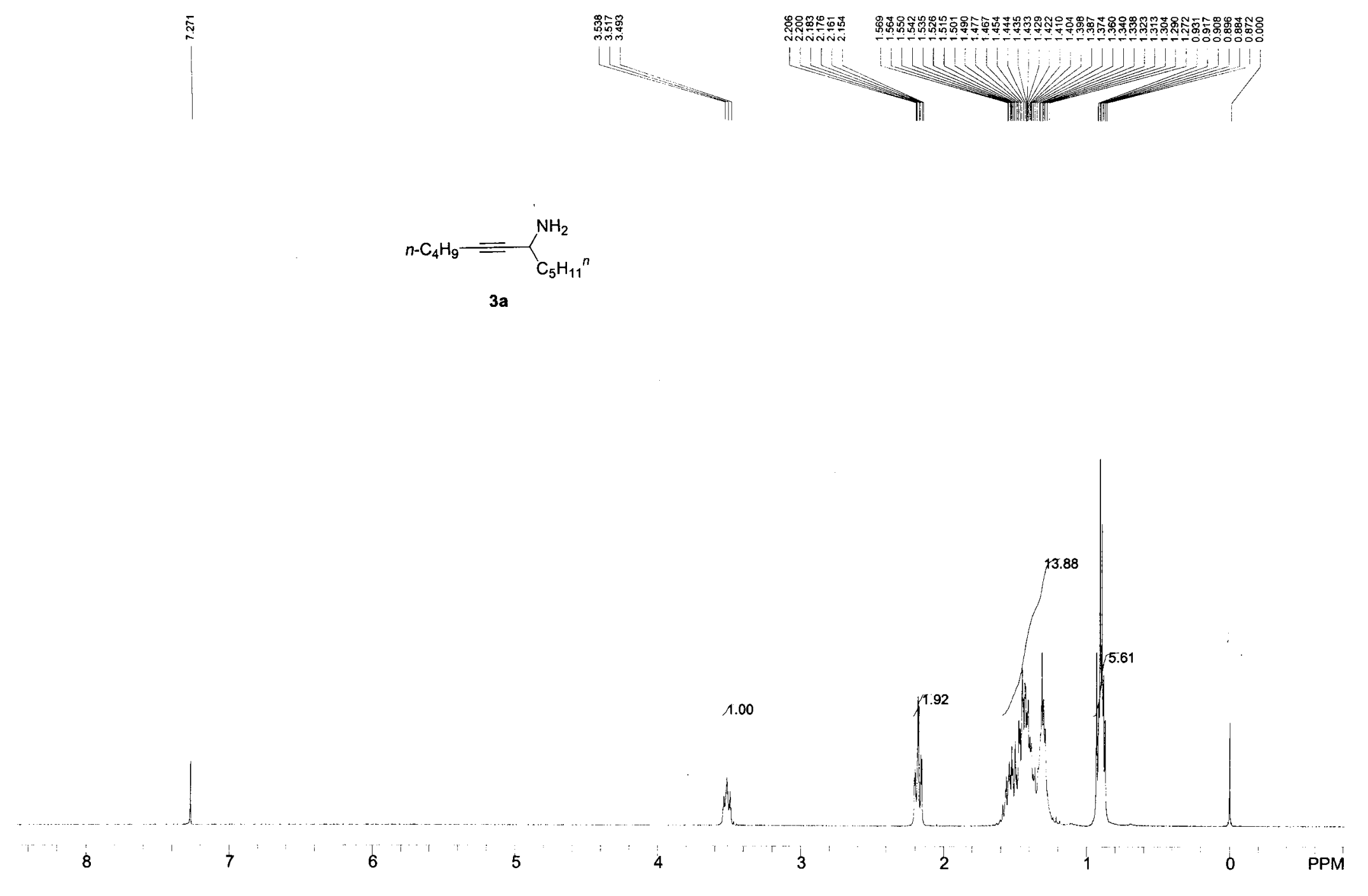
wb-7-171-2-c

Archive directory: /export/home/masm/vnmrsys/data

Sample direct
File: CARBON

Pulse Sequence: s2pul
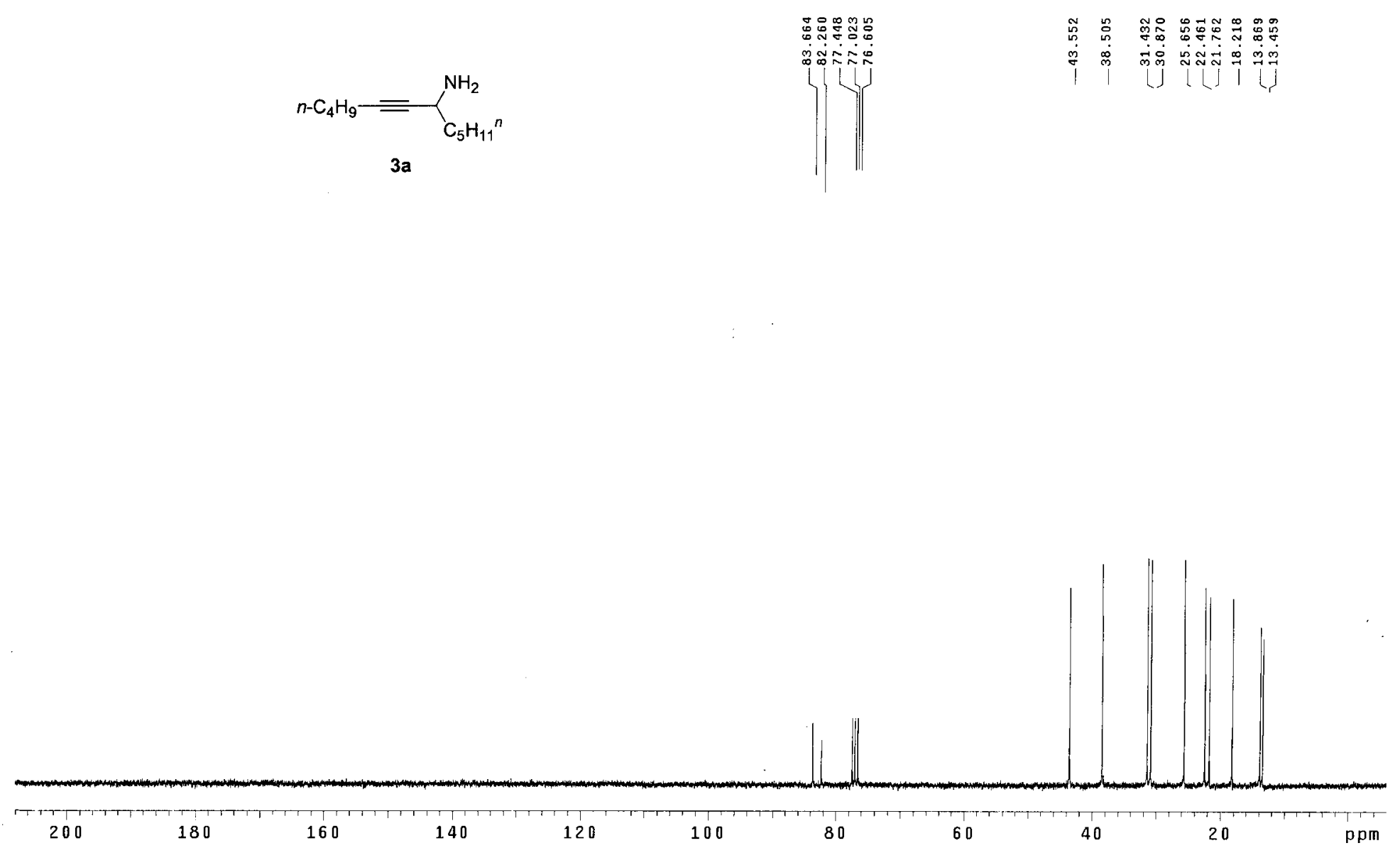


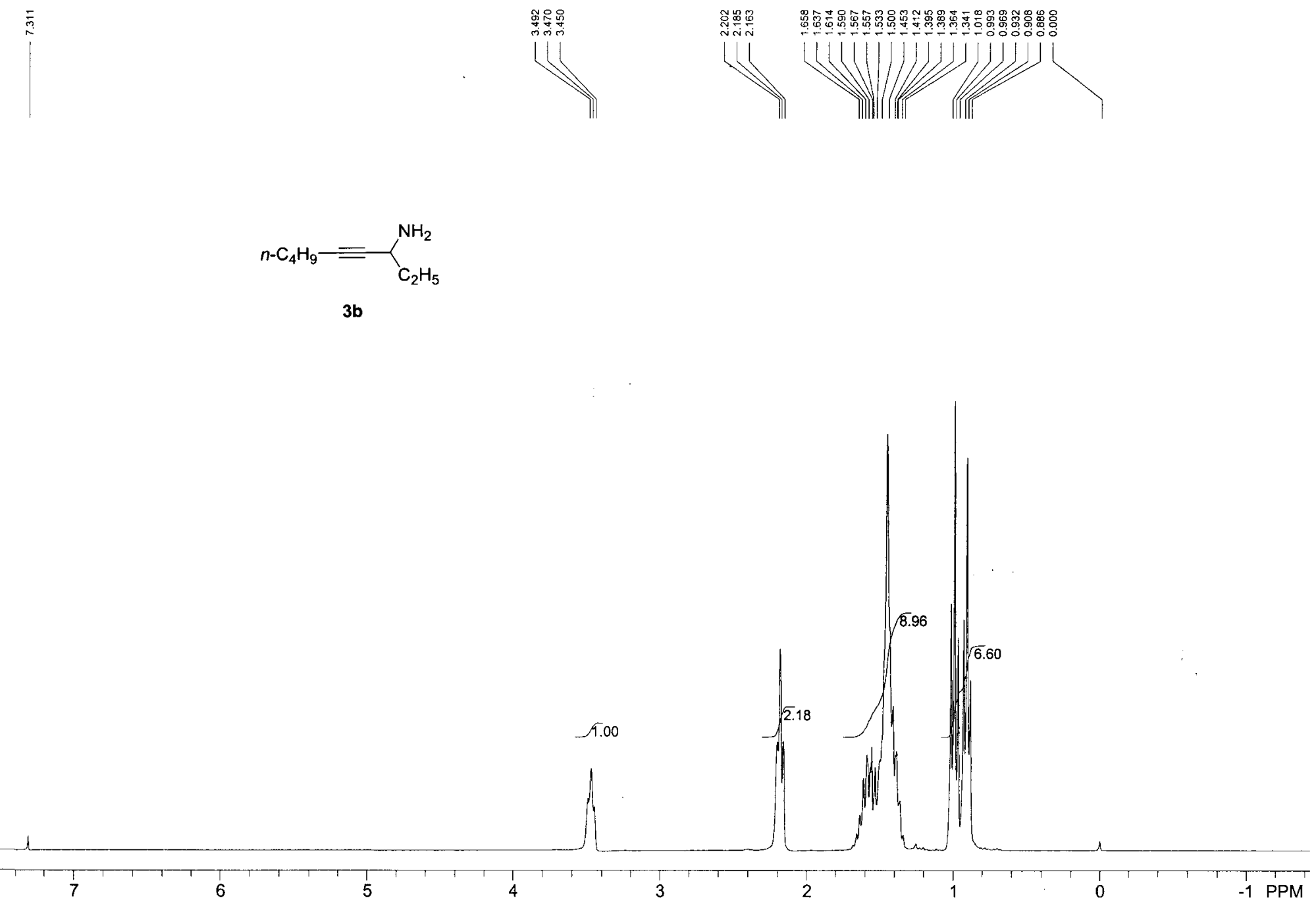


b-8-44-1

Archive directory: /export/home/masm/vnmrsys/data

Pulse Sequence: s2pul

$$
{ }_{n-\mathrm{C}_{4} \mathrm{H}_{9}}=-\mathrm{C}_{\mathrm{C}_{2} \mathrm{H}_{5}}^{\mathrm{NH}_{2}}
$$
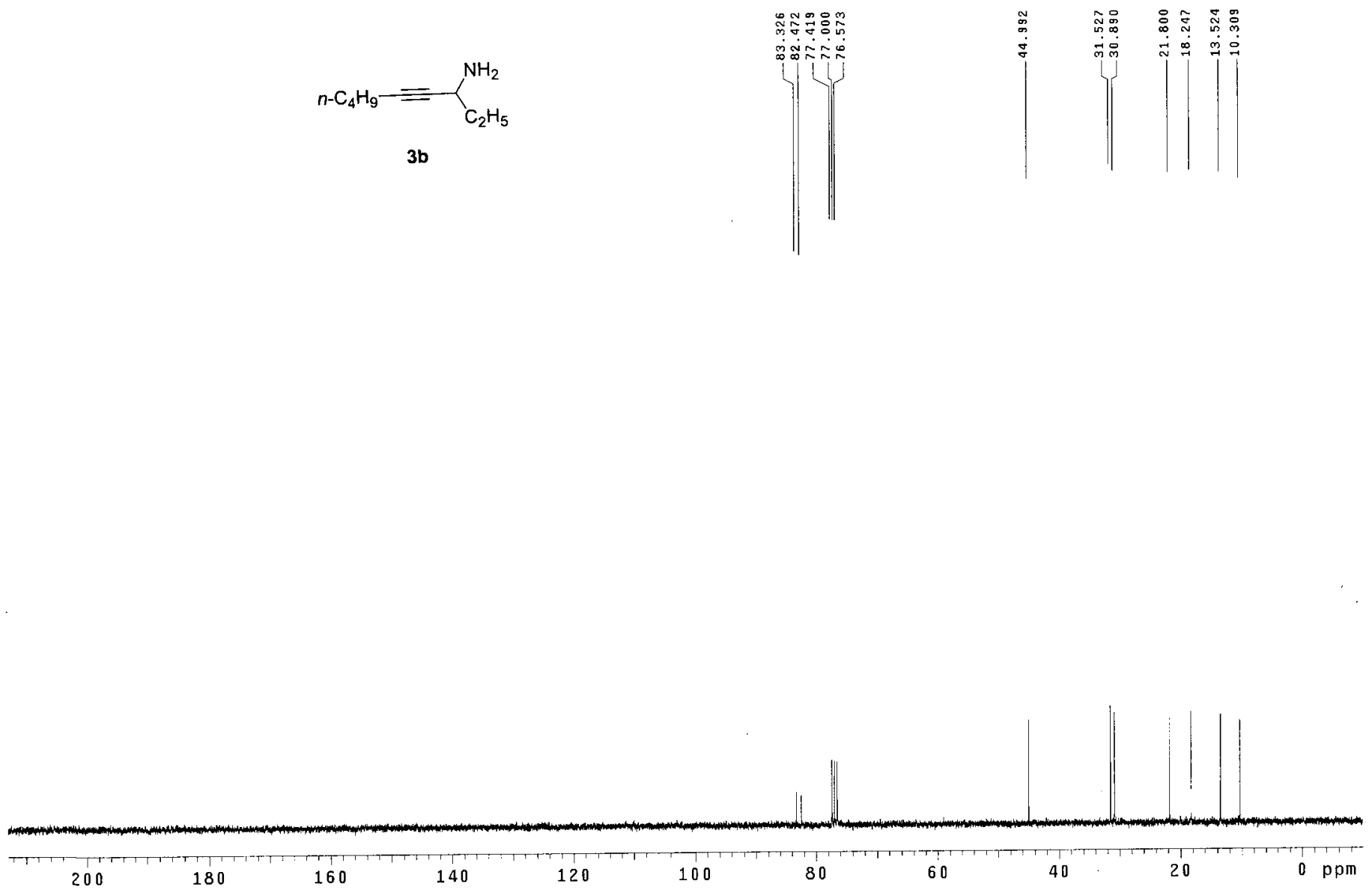


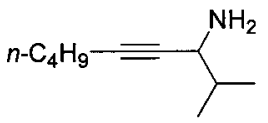
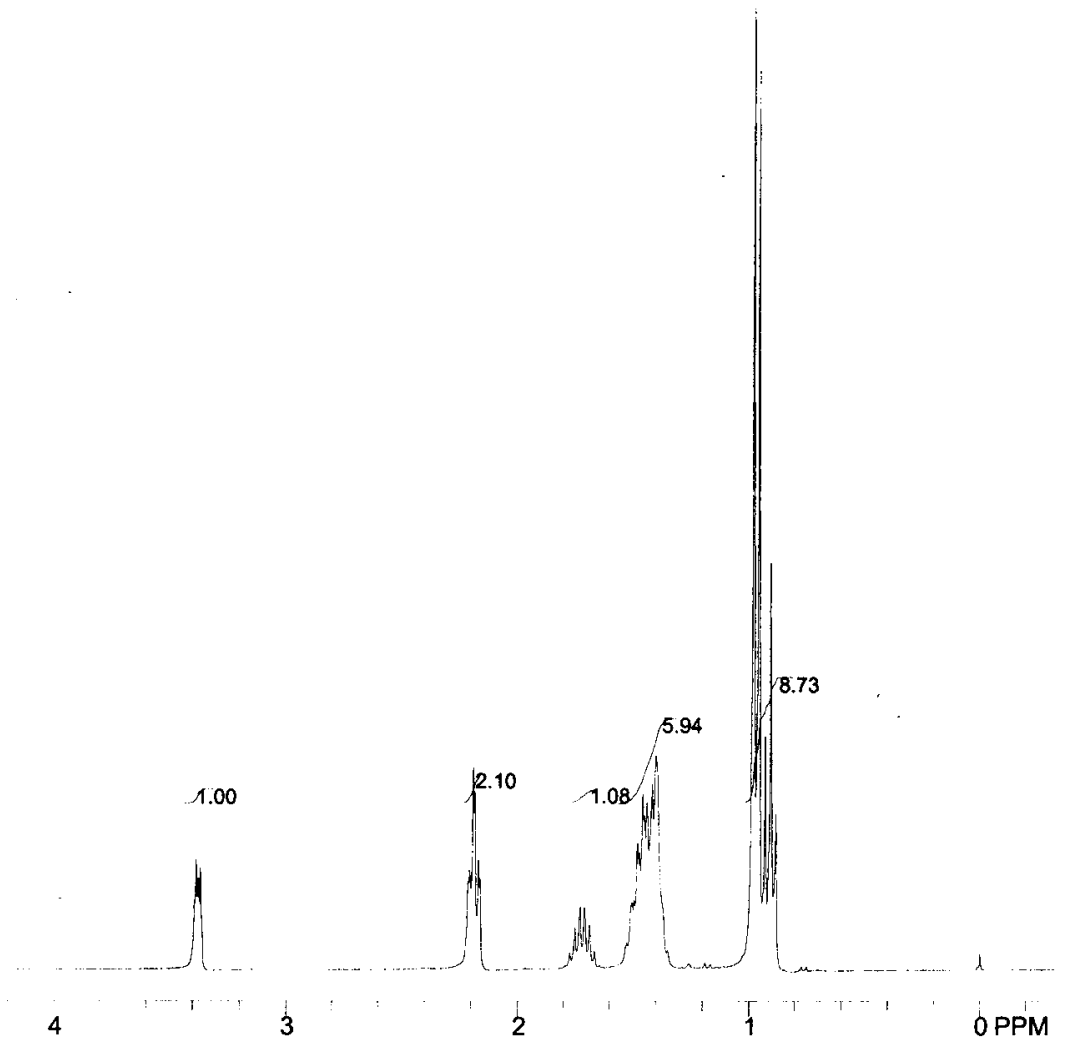
wb-8-21-1-c

Pulse Sequence: s2pur
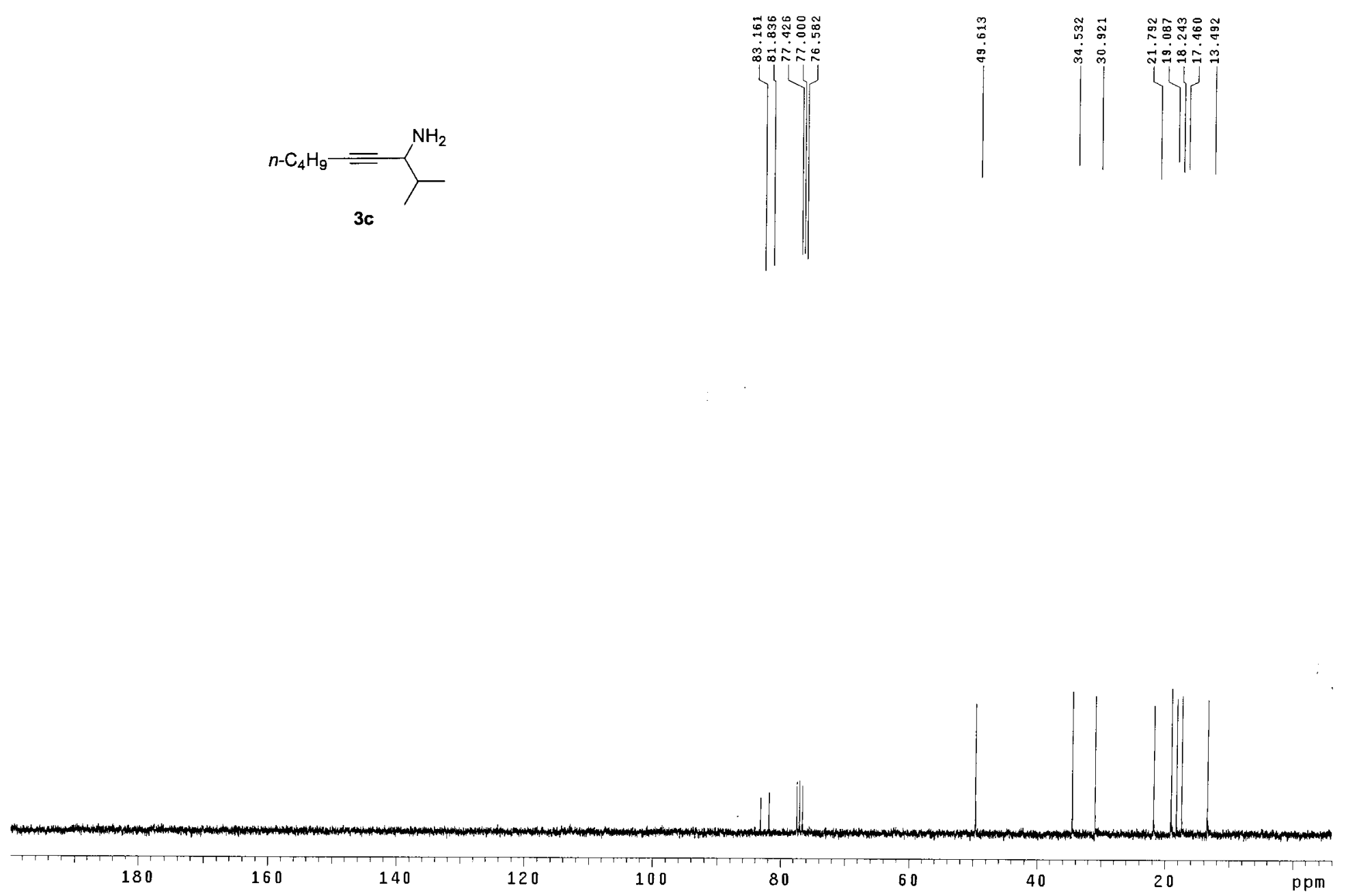

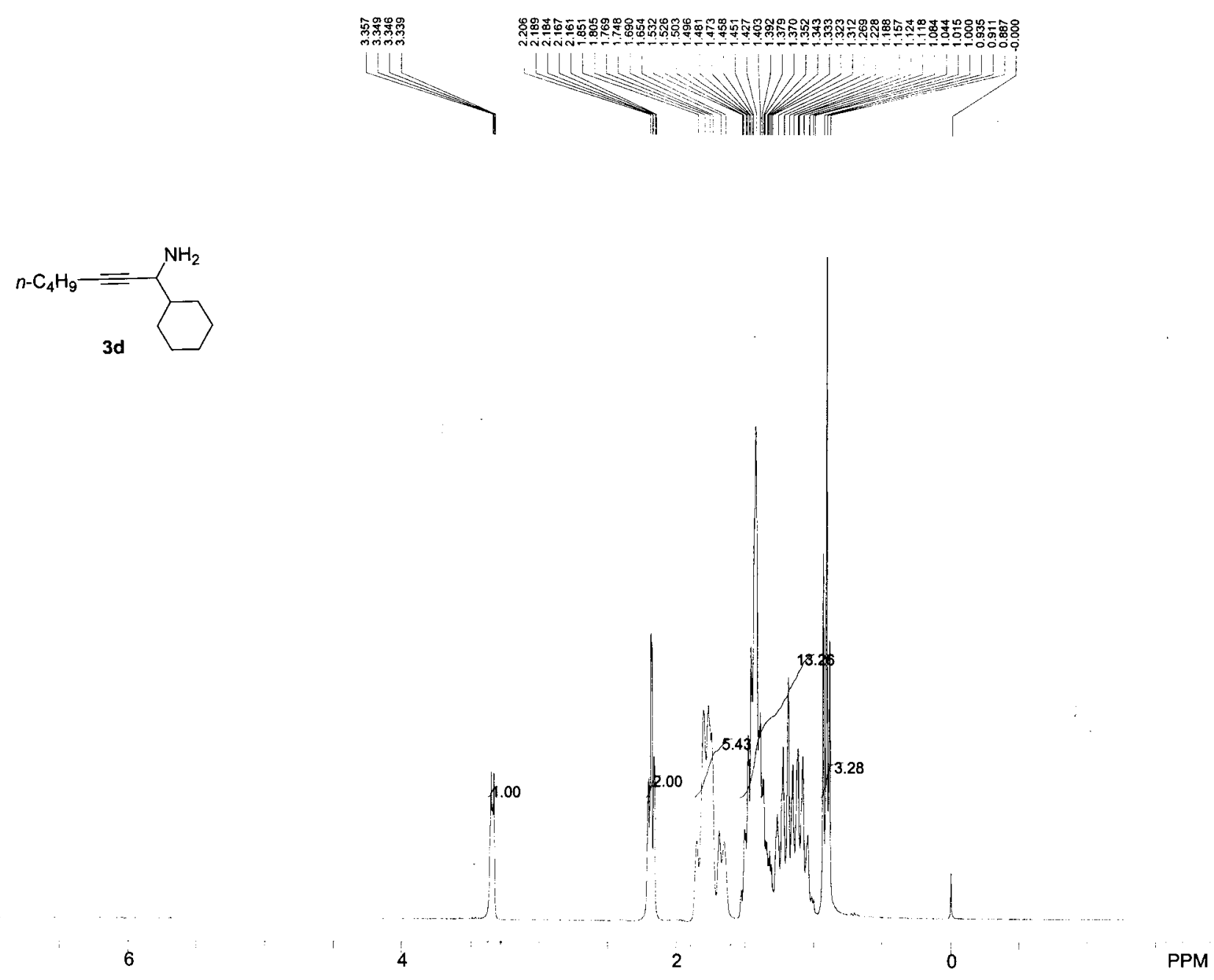
$w b-8-23-1-c$

Archive directory: /export/home/masm/unmrsys/data

Sample directory
file: CARBON

Pulse Sequence: s2pu

$$
{ }_{n-\mathrm{C}_{4} \mathrm{H}_{9}-}=\mathrm{\text {3d }}^{\mathrm{NH}_{2}}
$$

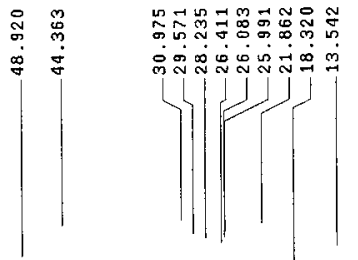

ำ

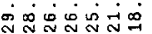

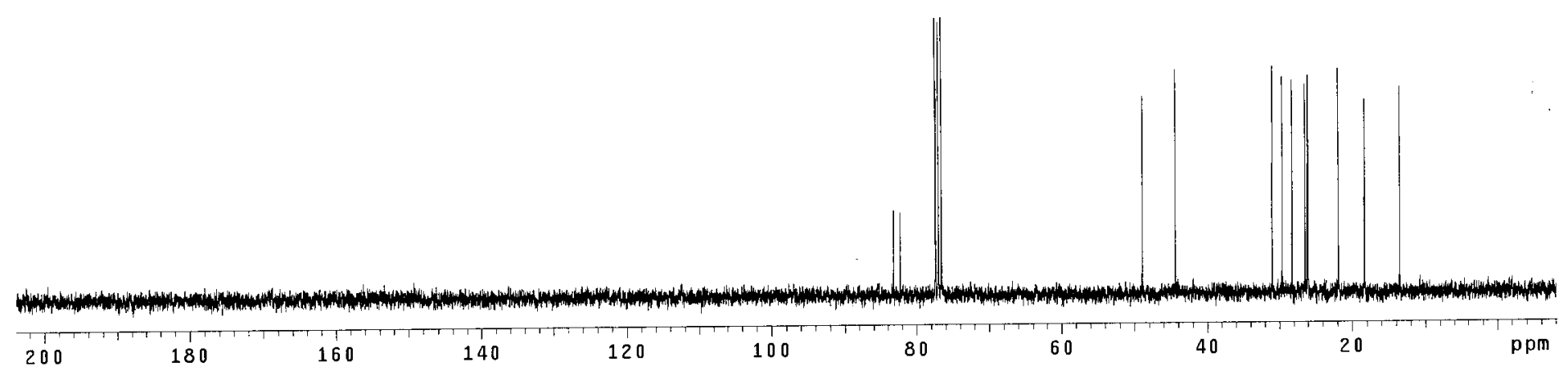




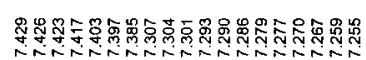

UMU

v

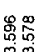

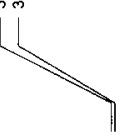

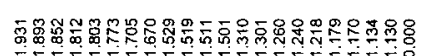

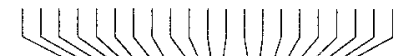

1.1) $y$

$\mathrm{Ph}=\square_{3 \mathrm{e}}^{\mathrm{NH}_{2}}$

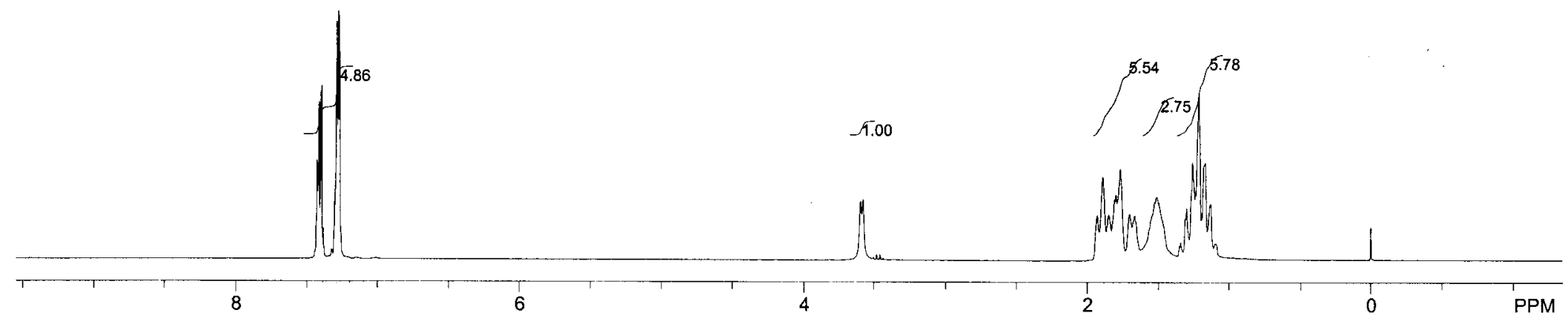


wb-8-25-1-c

Pulse Sequence: s2pul
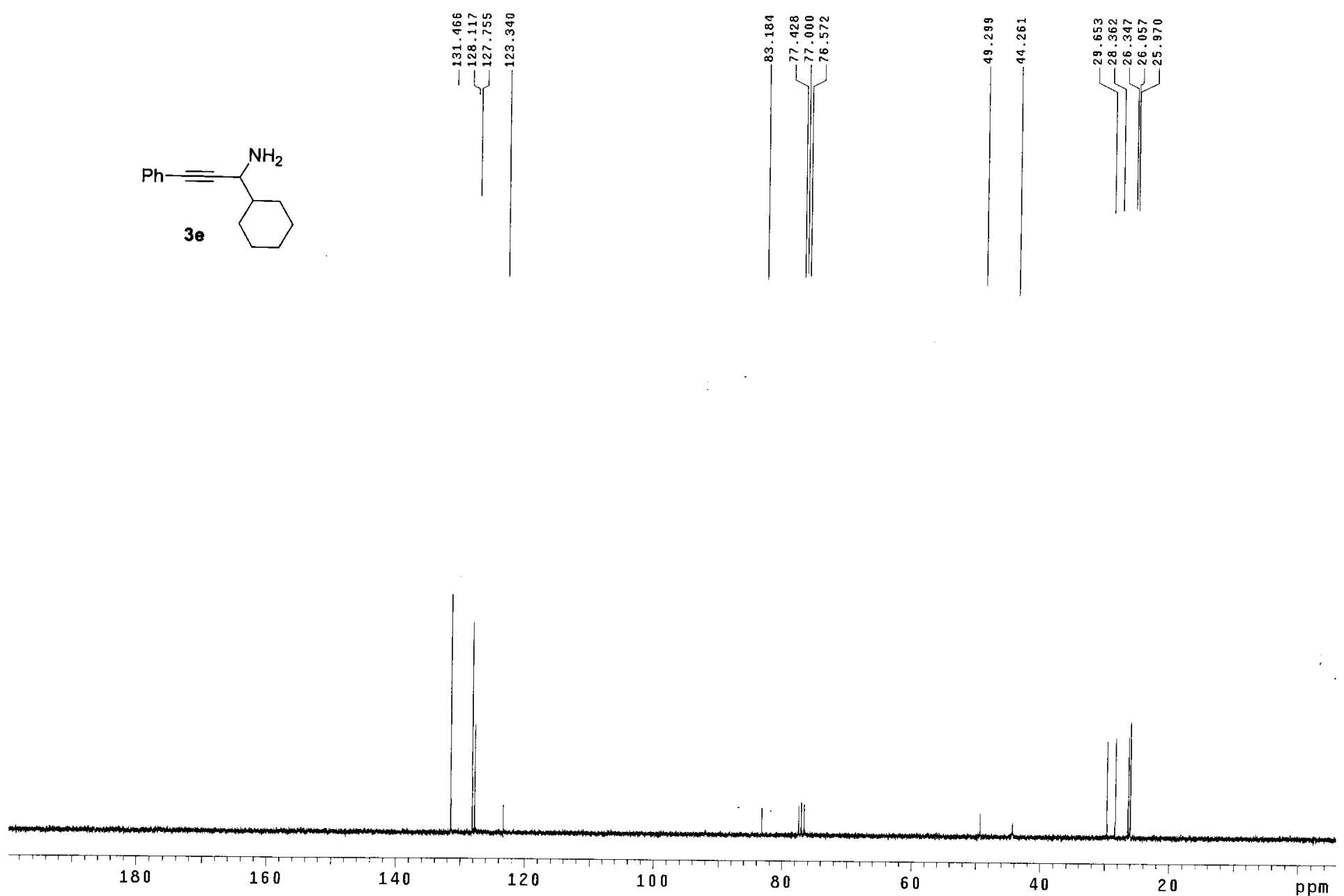


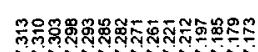

(1)

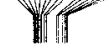

$\mathrm{Ph}=\mathcal{3 f}^{\mathrm{NH}_{2}}$

5.04

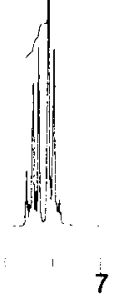

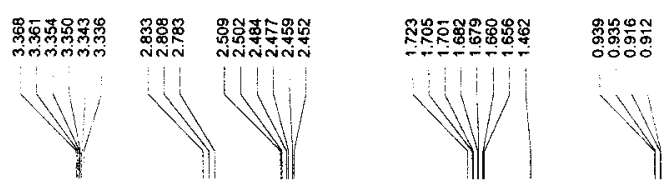

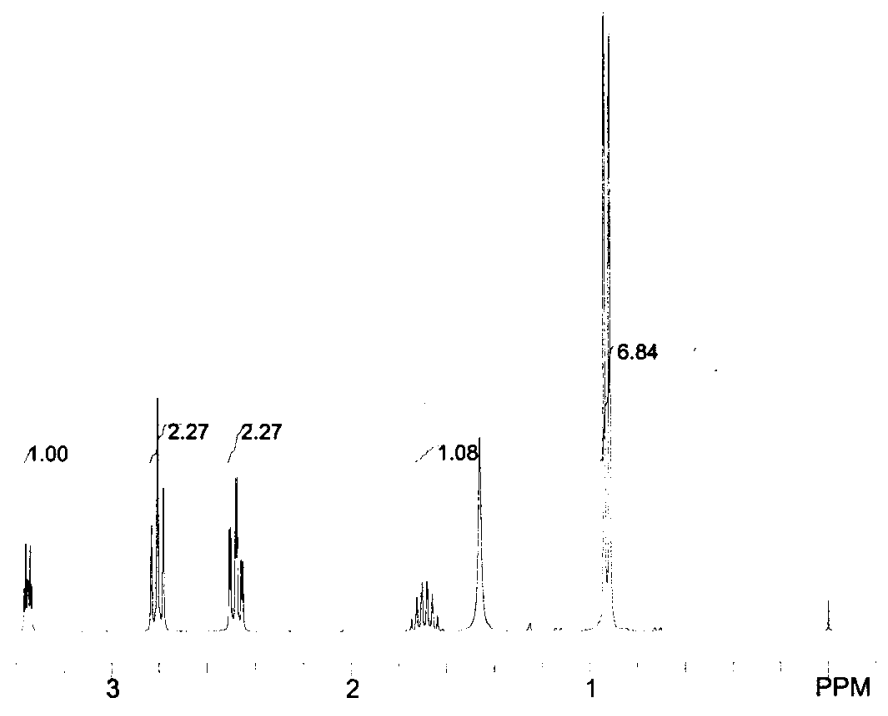



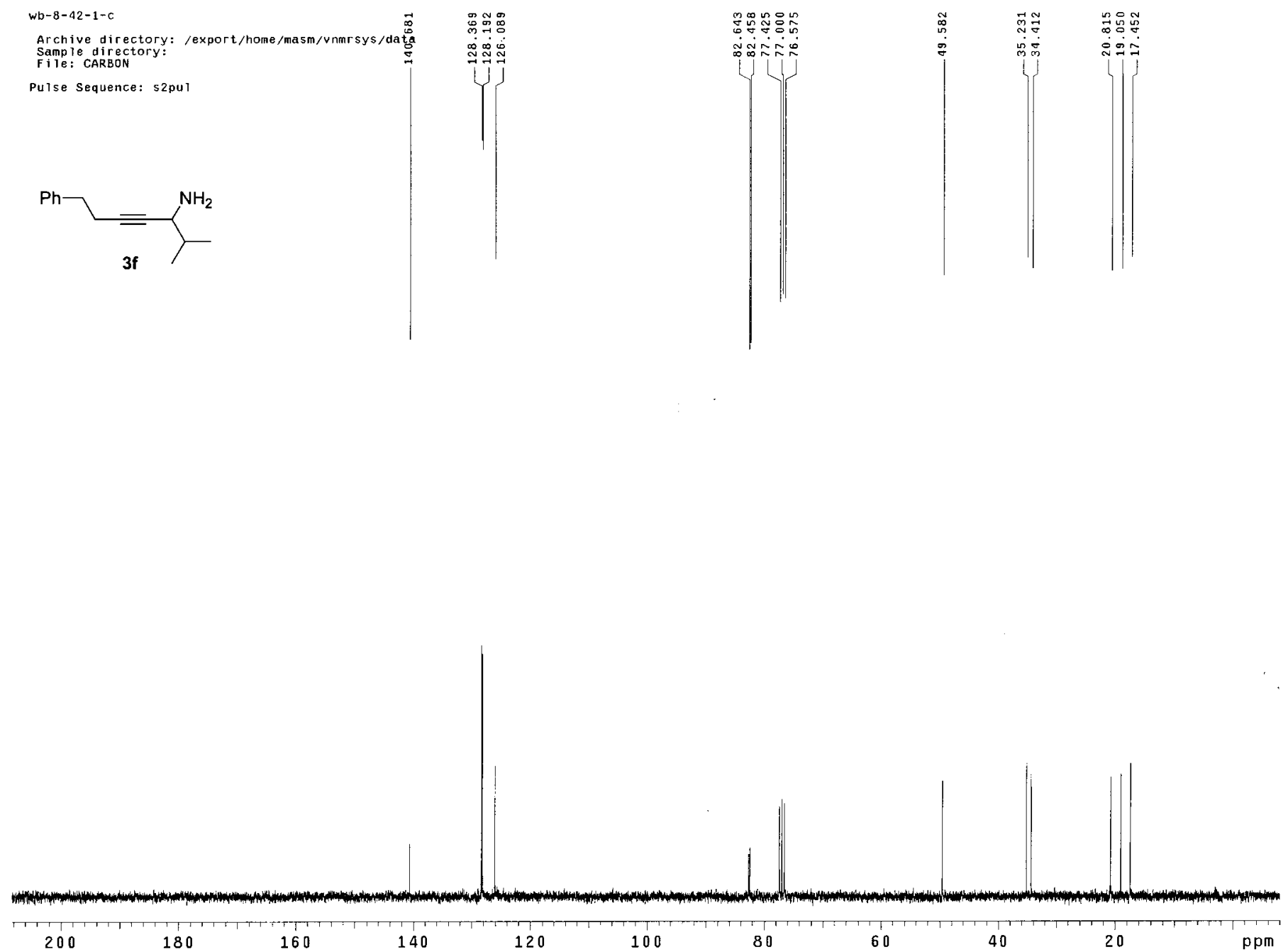
躁

$\mathrm{Bu}^{t}=-\chi^{\mathrm{Ng}}$

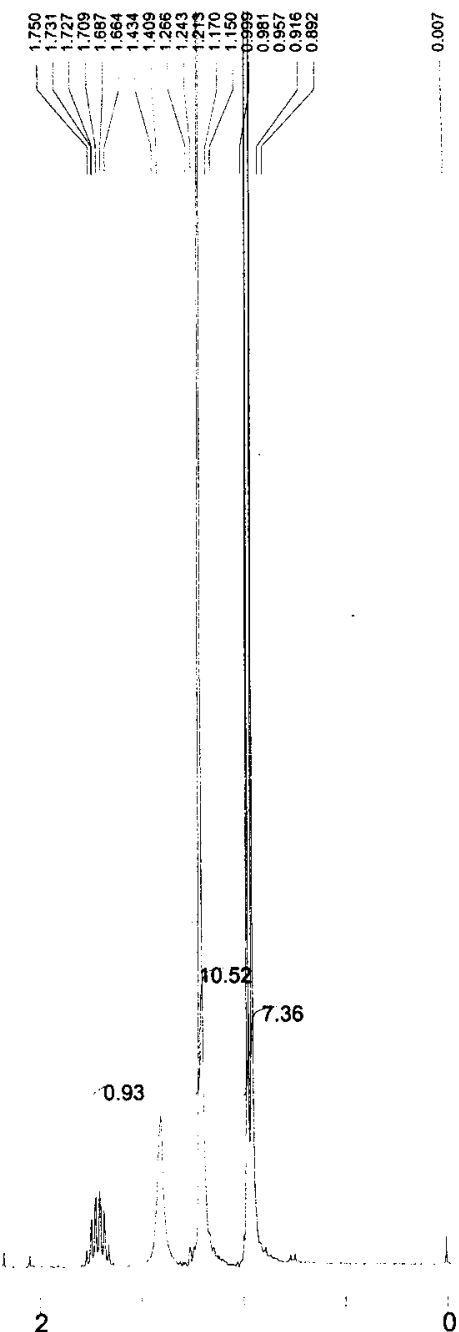

PPM 
Archive directory: /export/home/shi-m/vnmrsys/data

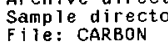

Pulse Sequence: s2pul

$$
\mathrm{Bu}^{\mathrm{t}}=\mathrm{J}_{\mathbf{3 g}}^{\mathrm{NH}_{2}}
$$
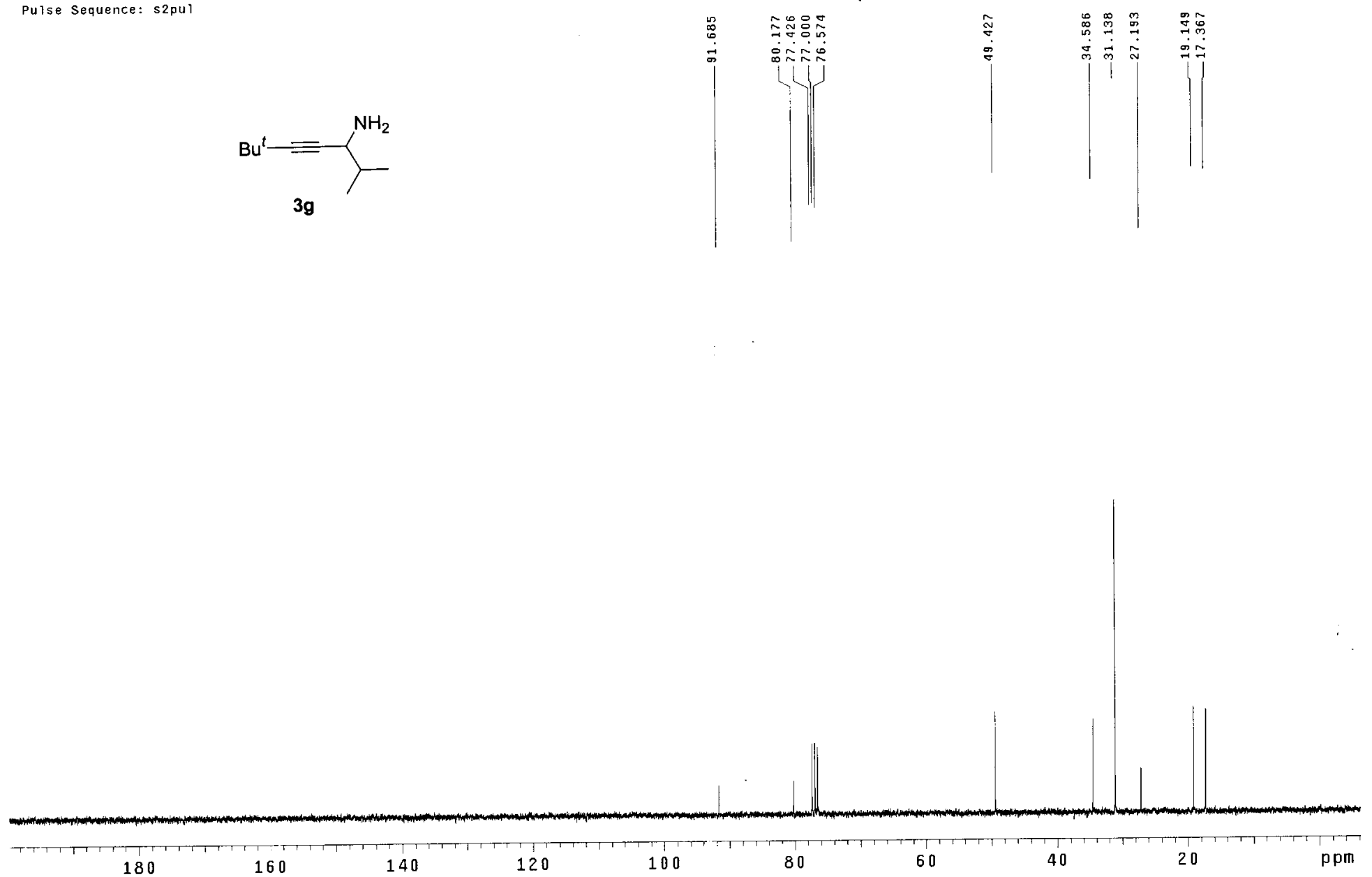

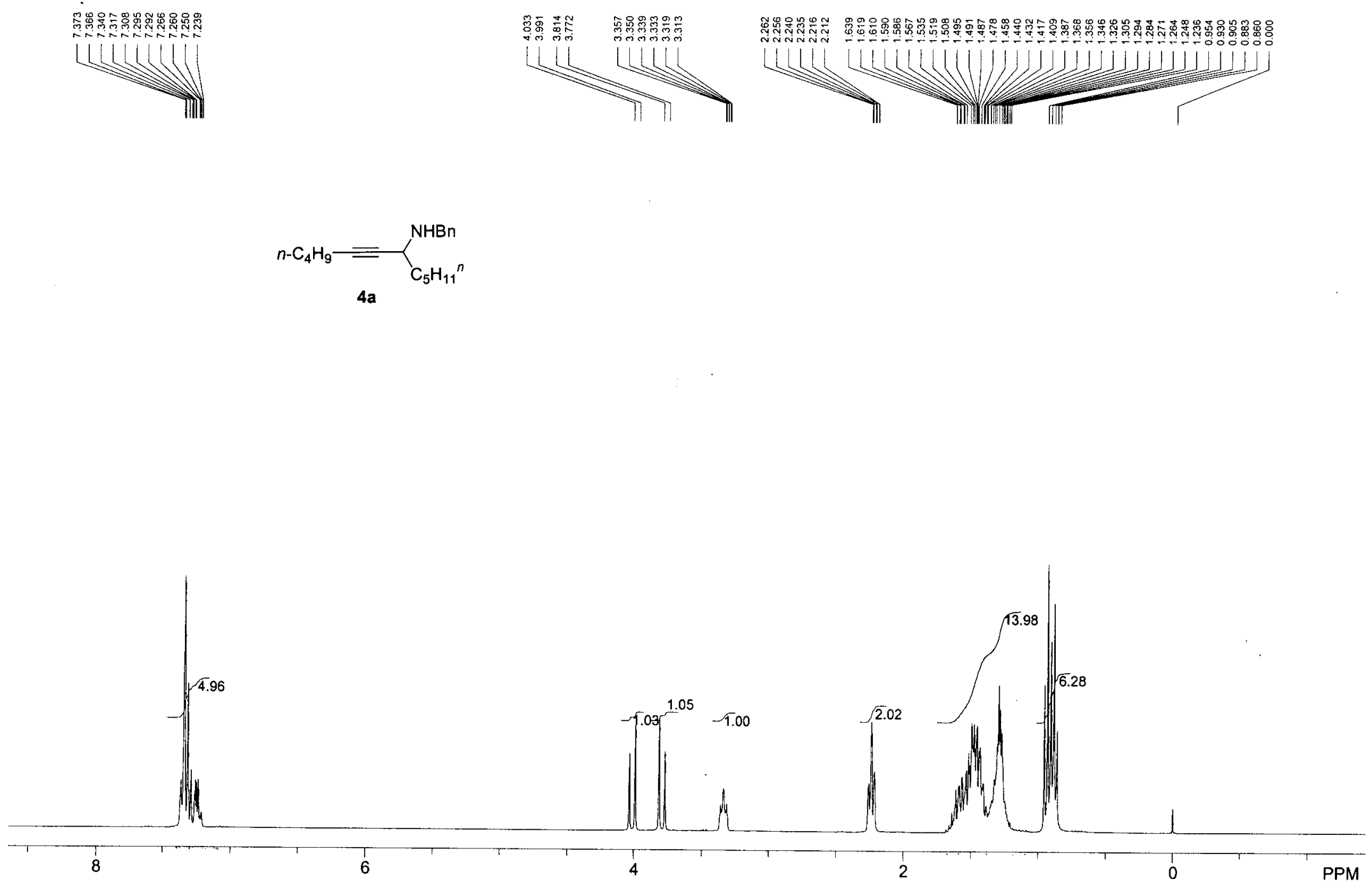
wb-8-69-c
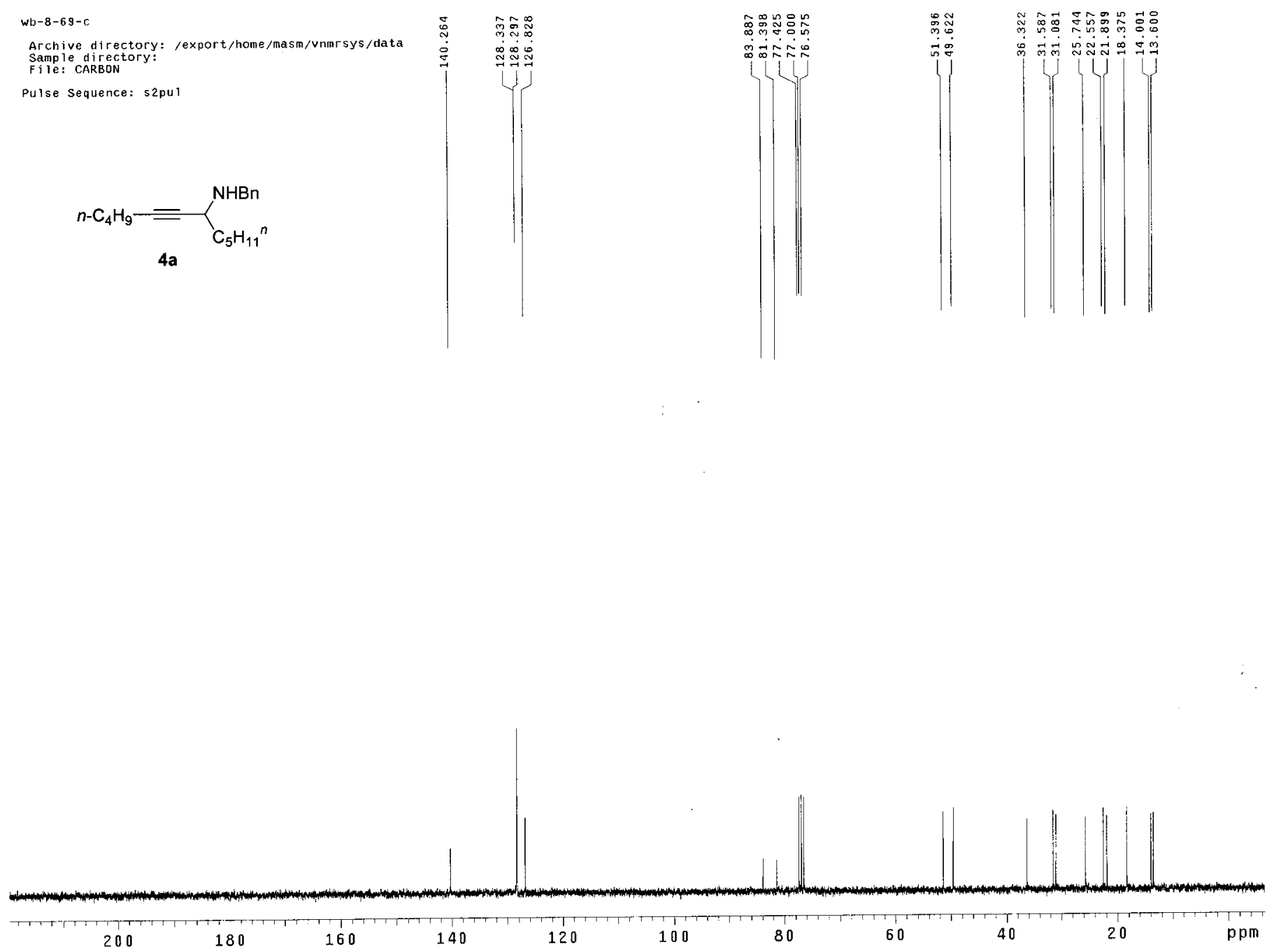

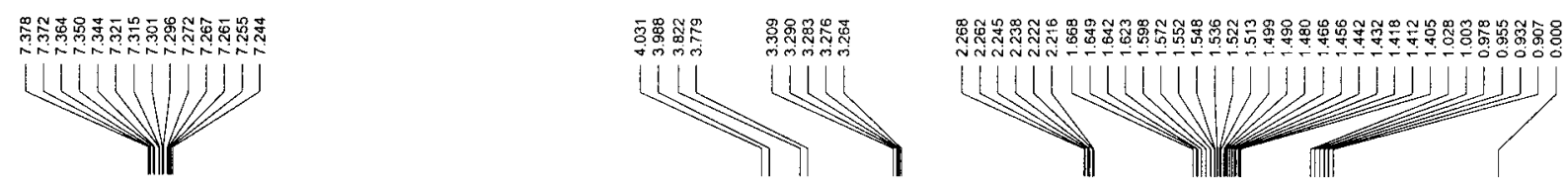

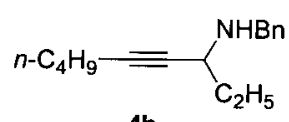
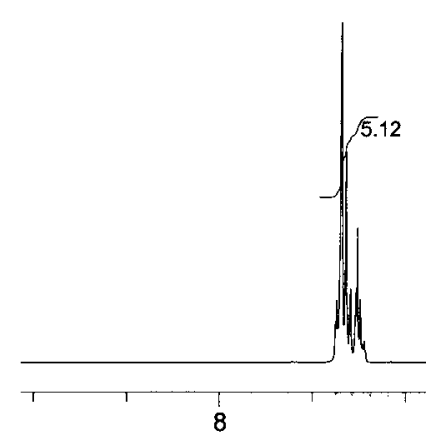

5.12 

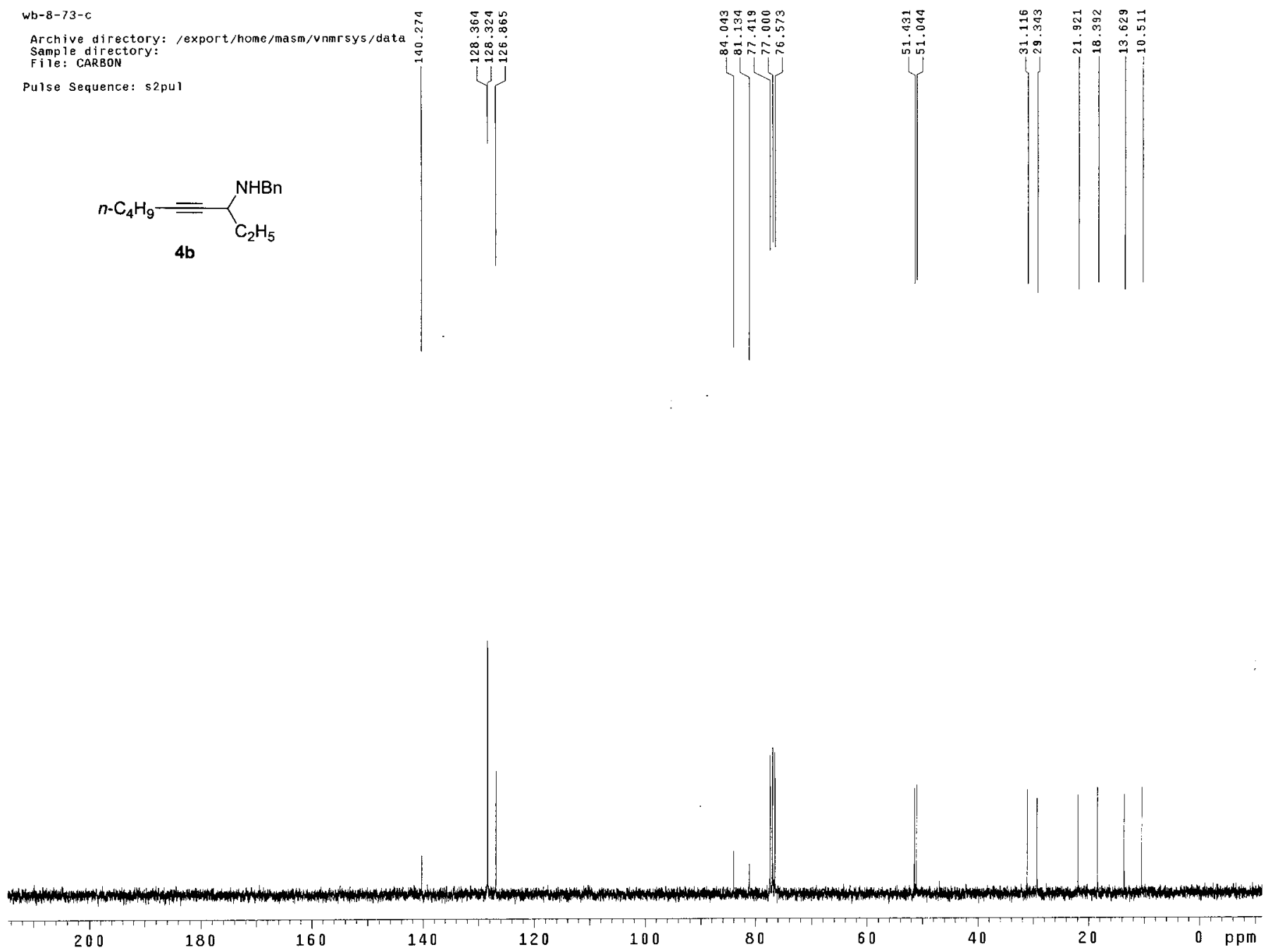
ำ

- LUUL

y.

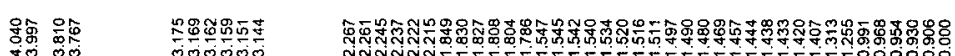

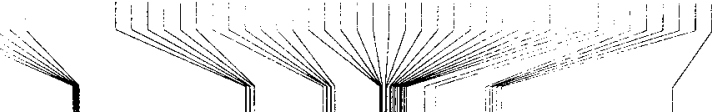

$n-\mathrm{C}_{4} \mathrm{H}_{9}=\sum_{-}^{\mathrm{NHBn}}$

4c

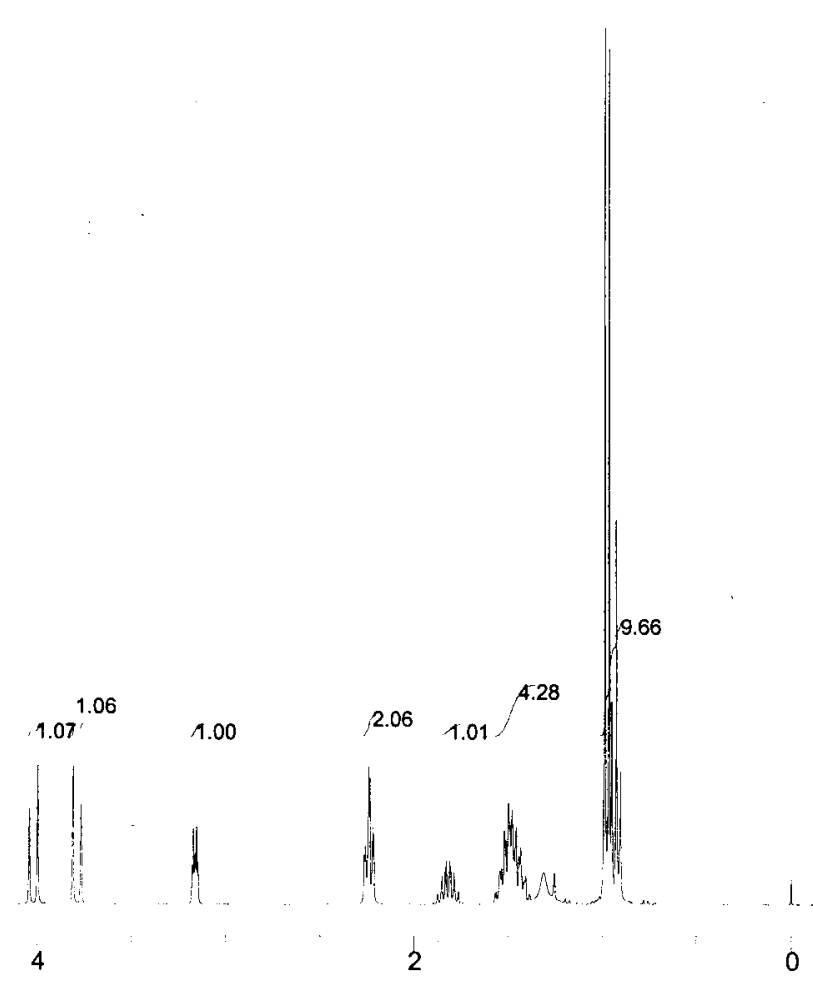


wb-8-38-c13

Archive directory: /export/home/masm/vnmrsys/data

File: CARBON

Pulse Sequence: s2pu
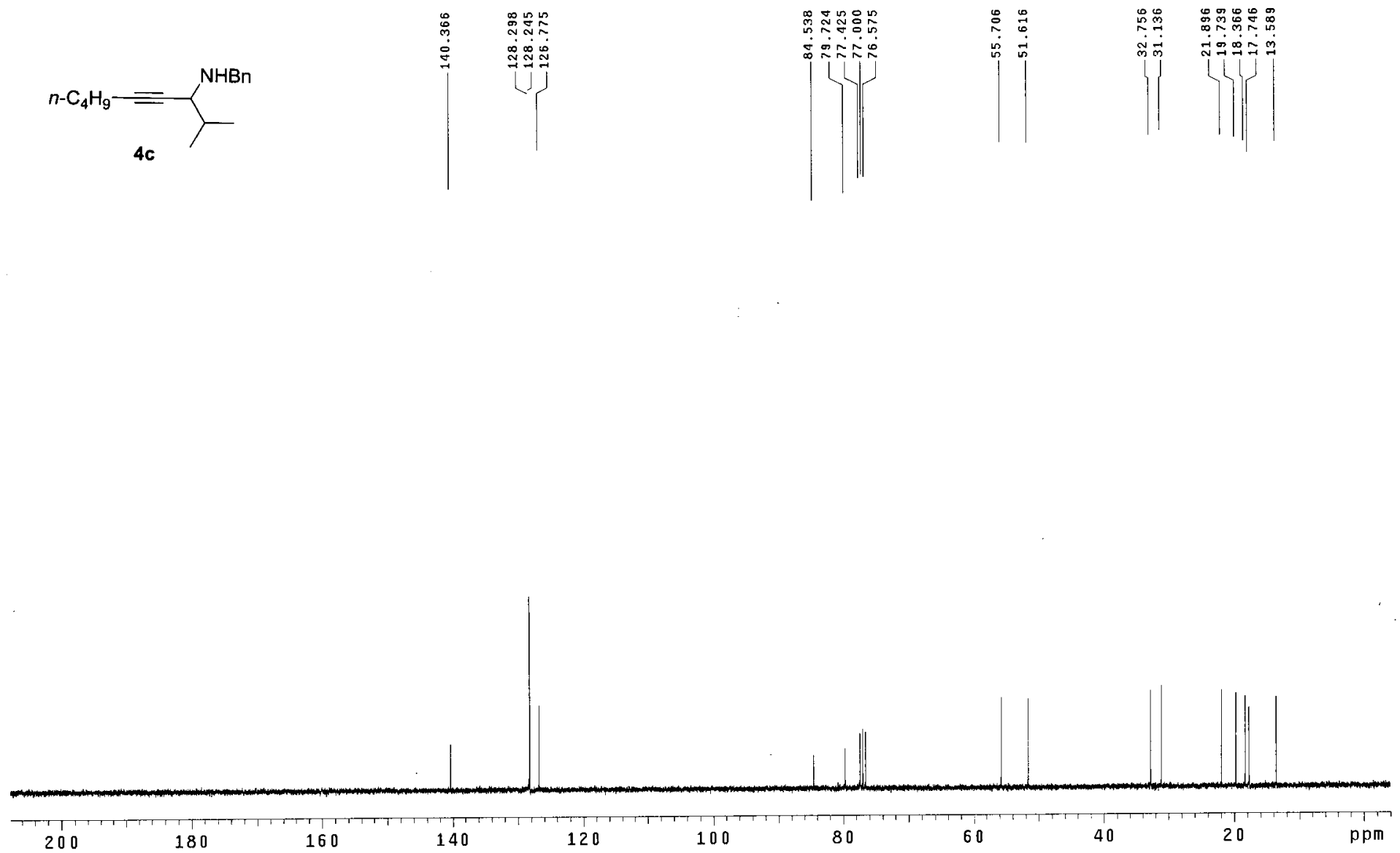

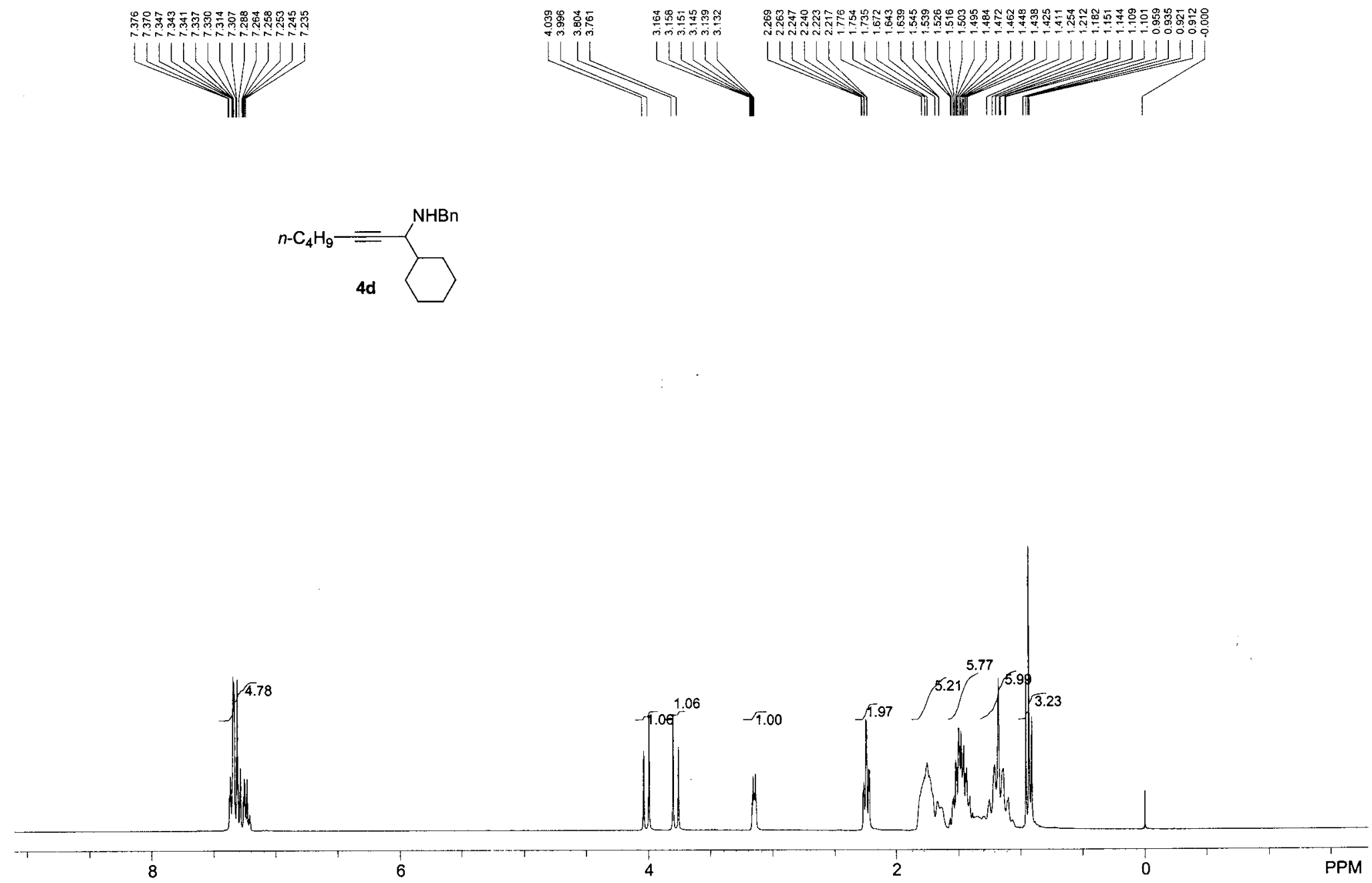


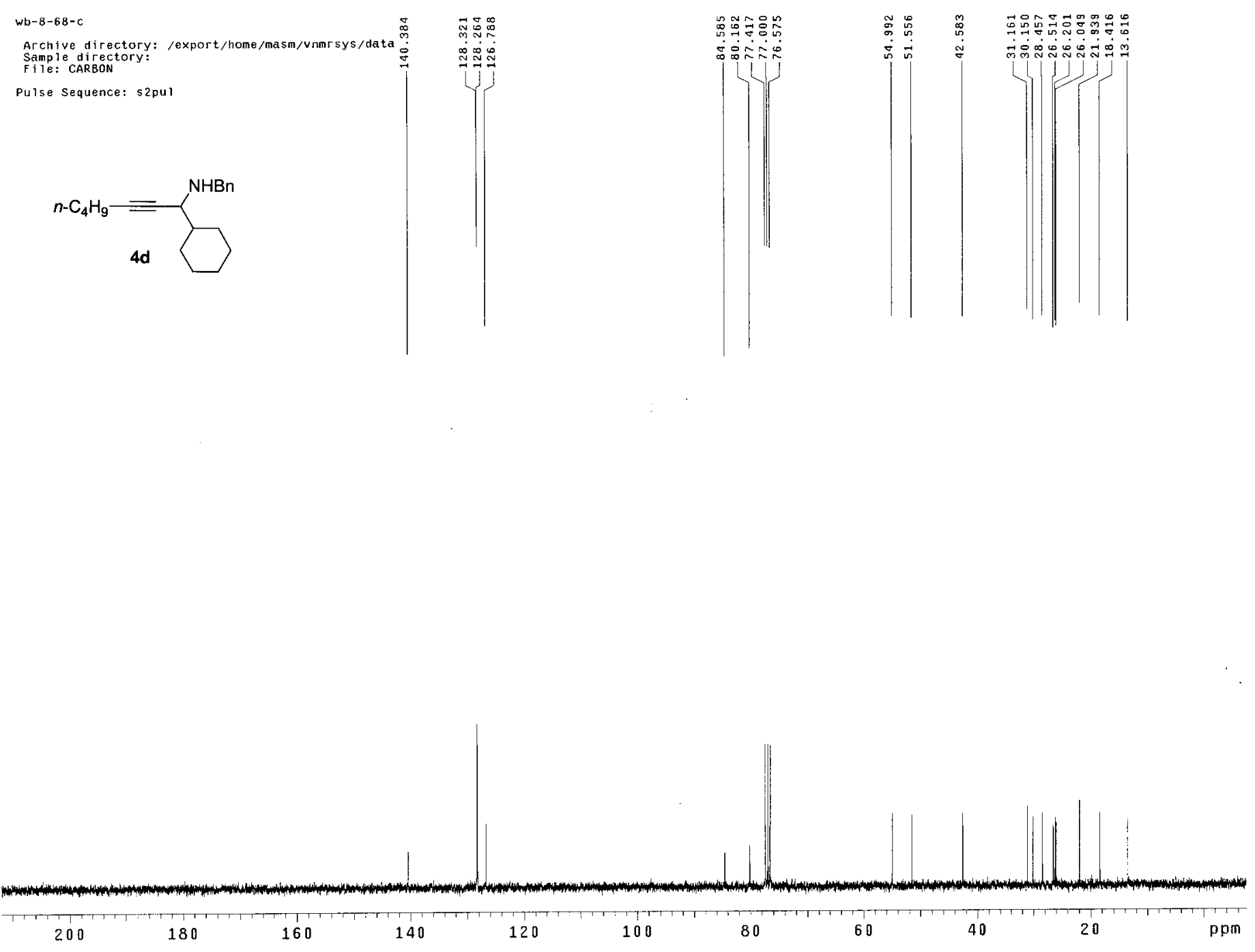




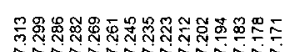

(1)IIJ)JJ)

,
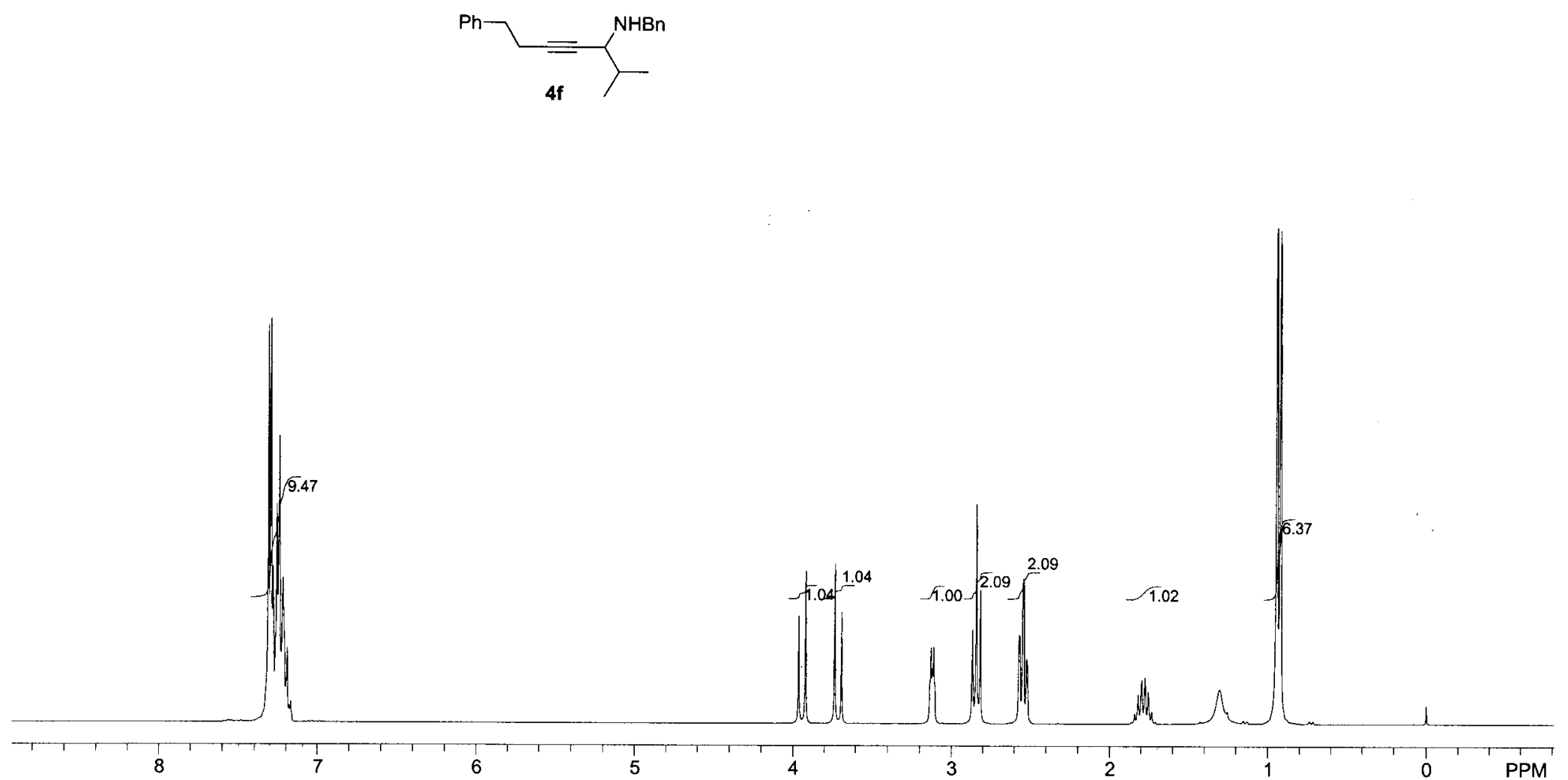


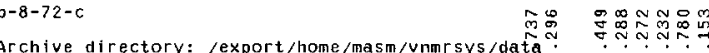

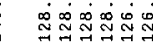

Pulse Sequence: s2pul

$\mathrm{Ph}-$

$=\gamma_{4 f}^{N H B n}$
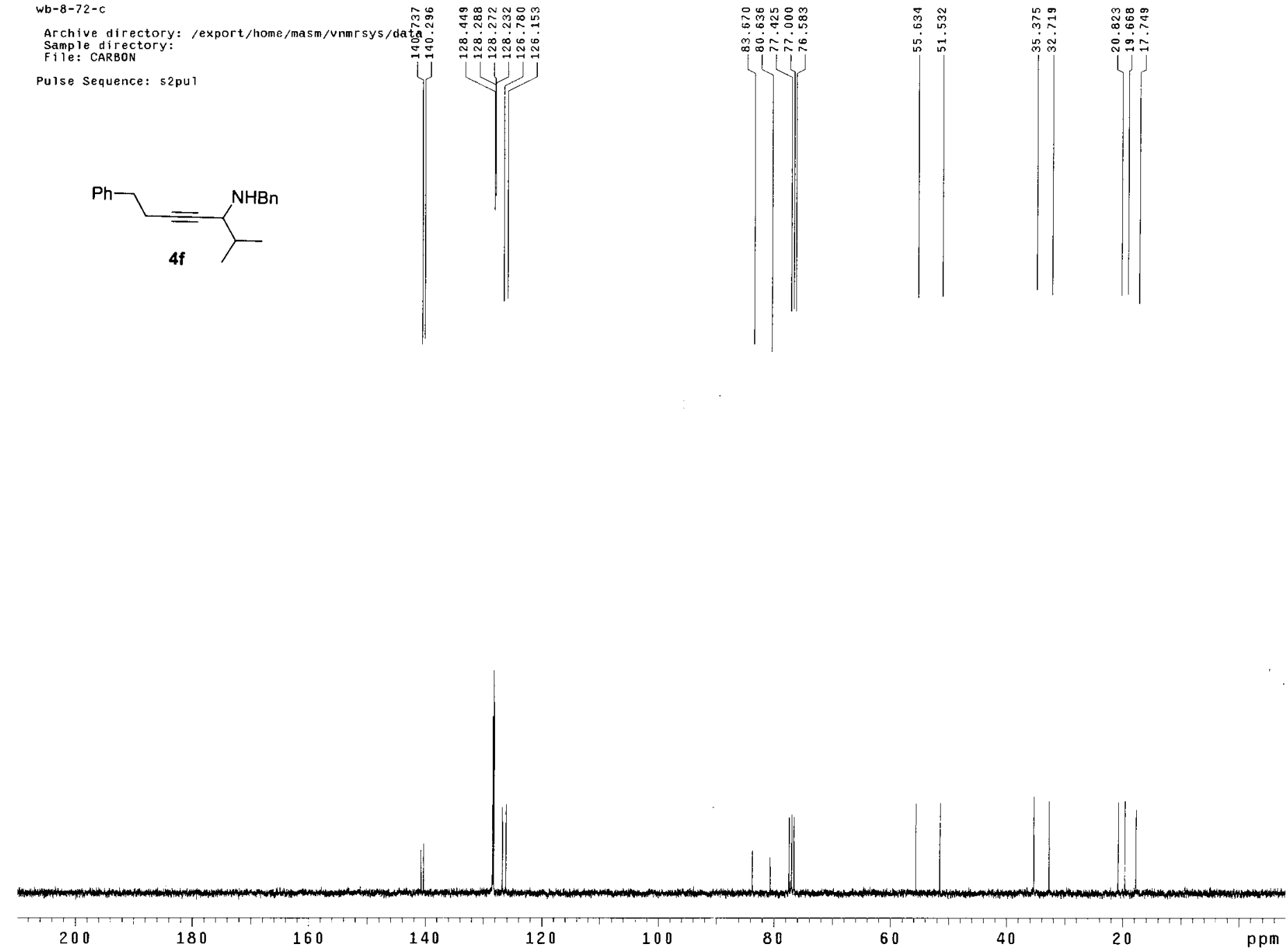


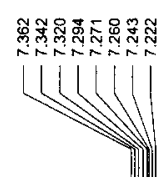

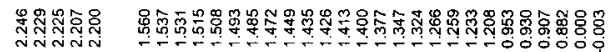

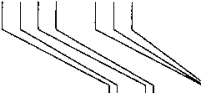

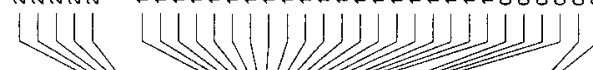

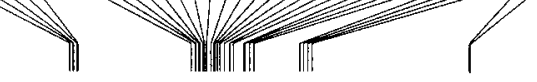

${ }_{n-\mathrm{Bu}}=<_{4 \mathrm{~h}}^{\mathrm{NHBn}}$

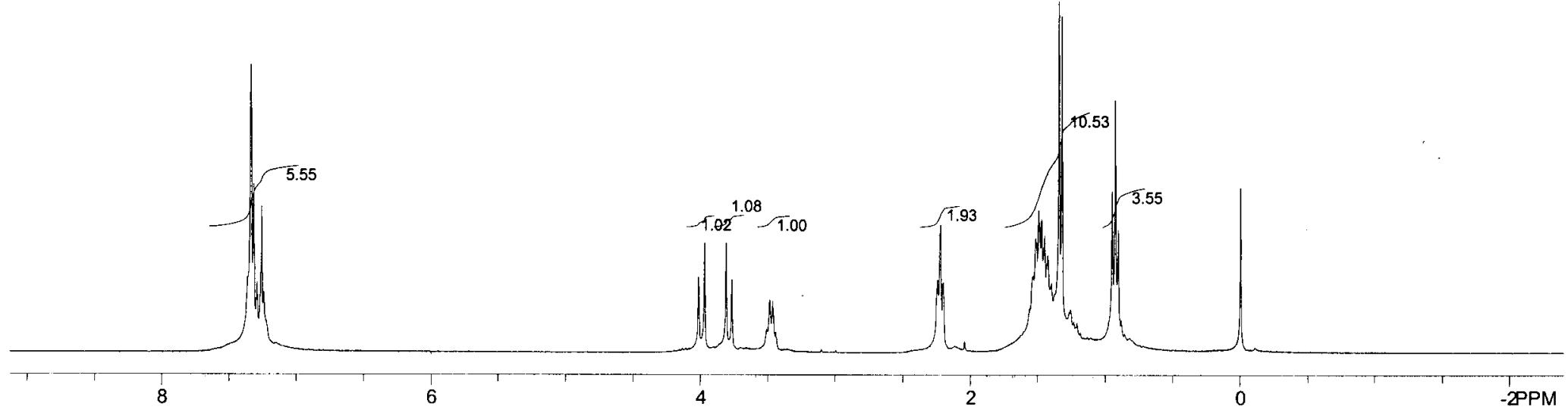


wb-8-4-c

Archive directory: /export/home/masm/vnmrsys/data

Sample directory:
File: CARBON

Pulse Sequence: s2pul

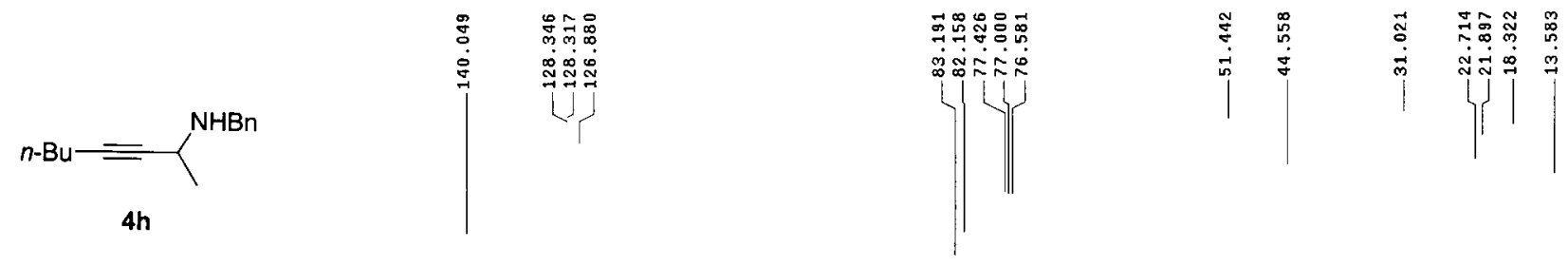

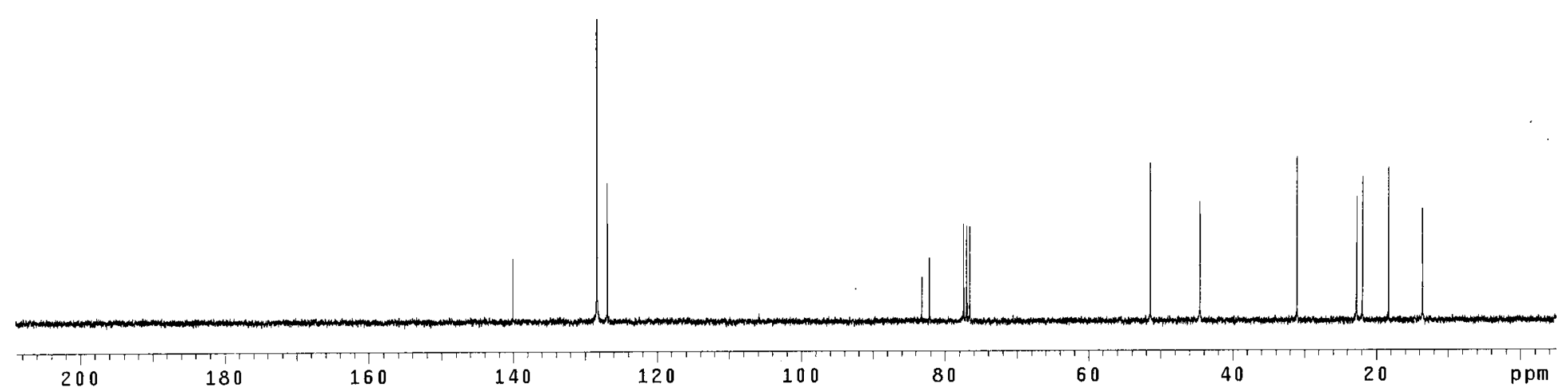



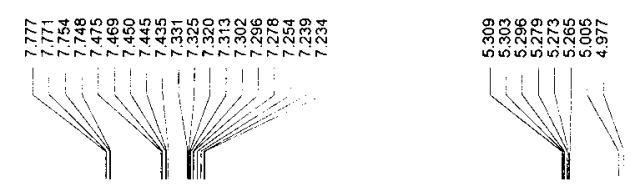

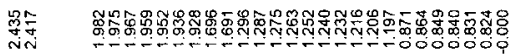

$n-\mathrm{C}_{4} \mathrm{H}_{9}=\mathcal{K}^{\mathrm{NHTS}}$

4I

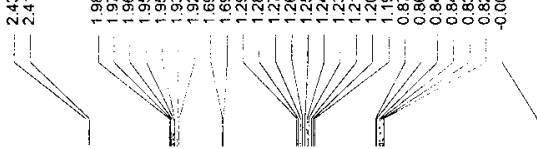

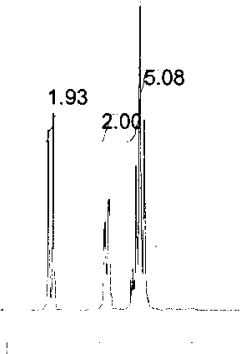

8
0.969 .00

(i)

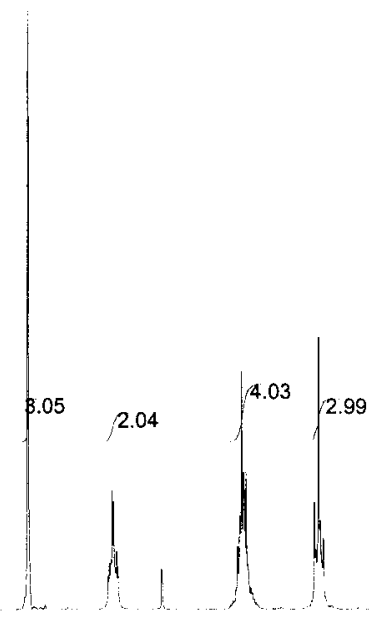

2 
wb-8-95-c

Archive directory: /export/home/masm/unmrsys/data

File: CARBON

Pu 1se Sequence: $52 \mathrm{pu} 1$
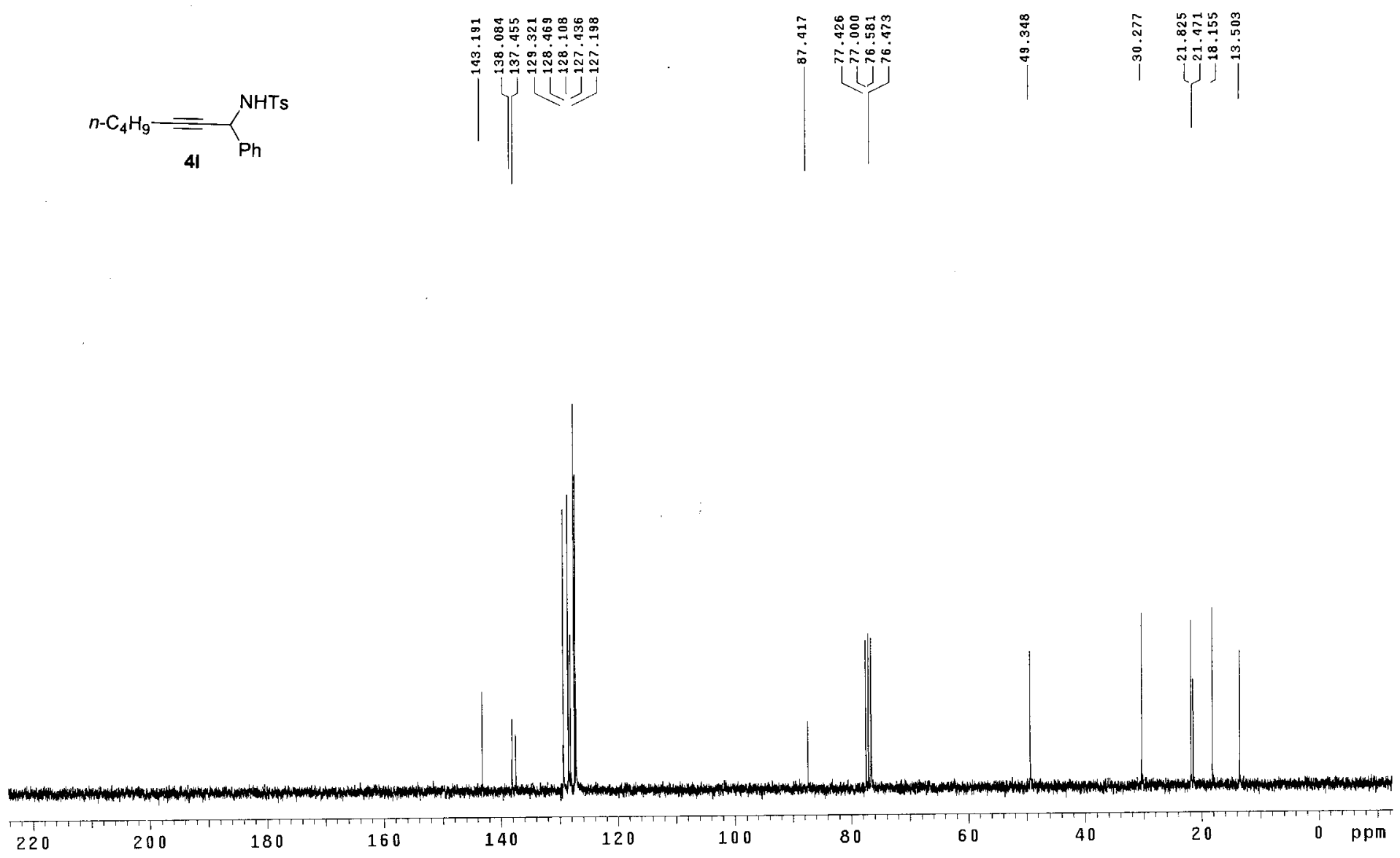


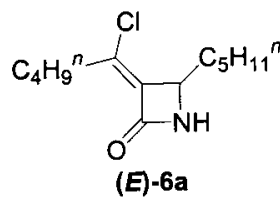

1.00

1.00

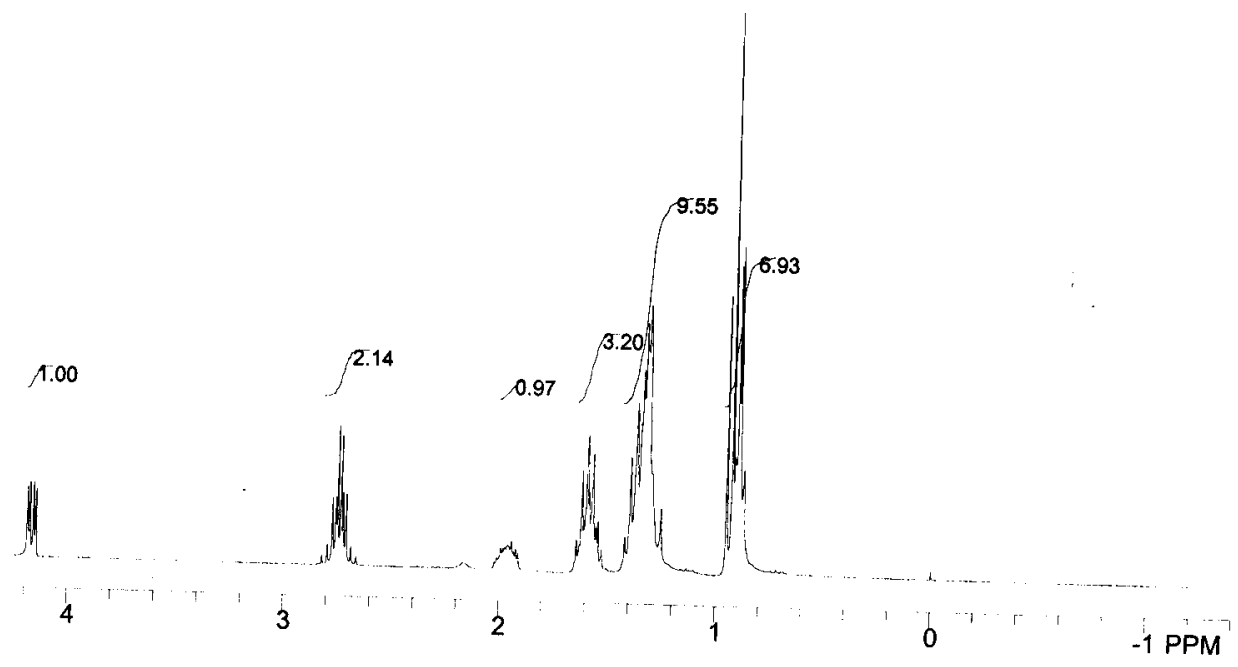


wb-7-174-c

Archive directory: /export/home/masm/vnmrsys/data

Sample director
File: CARBON

Pulse Sequence: s2pul
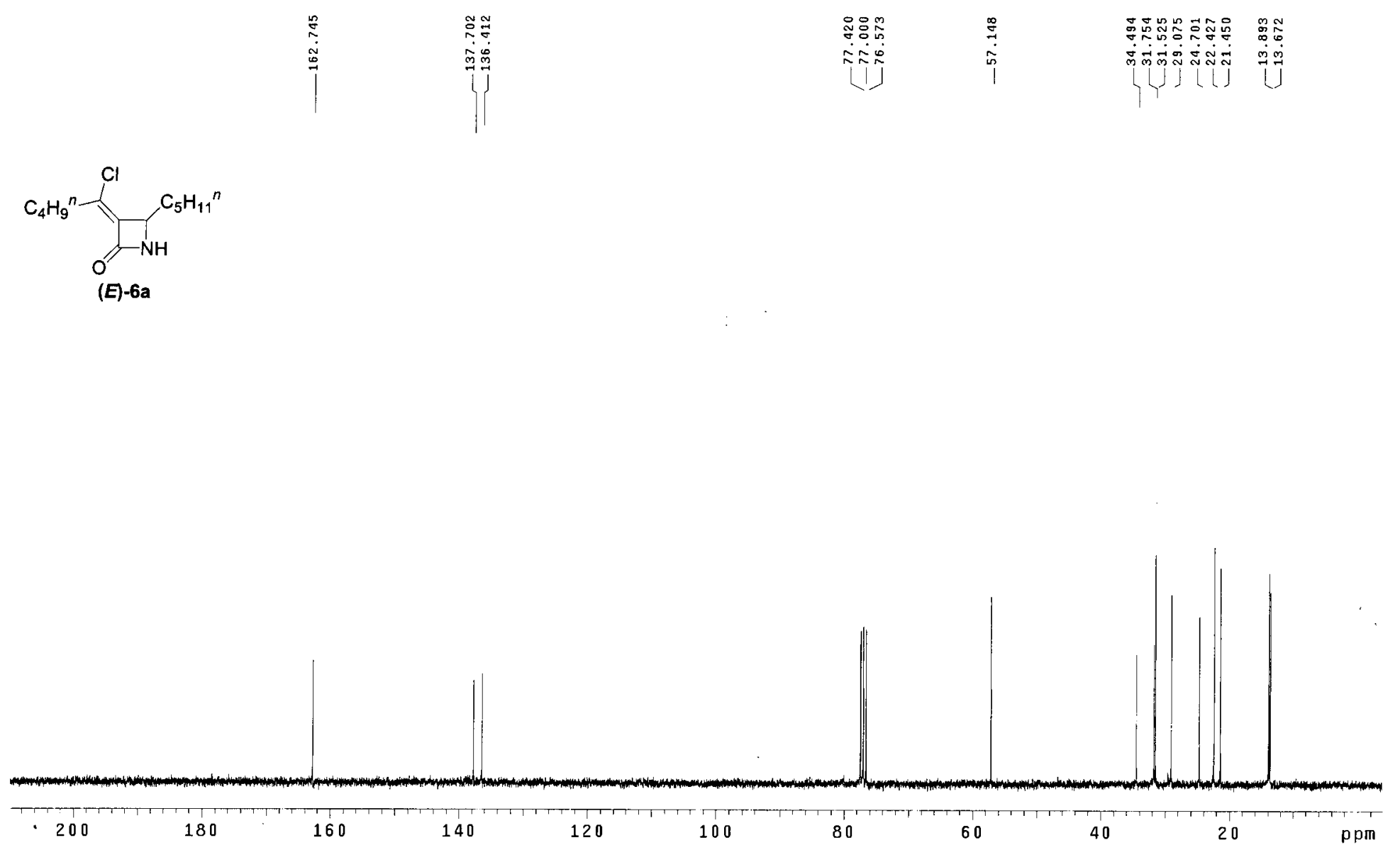

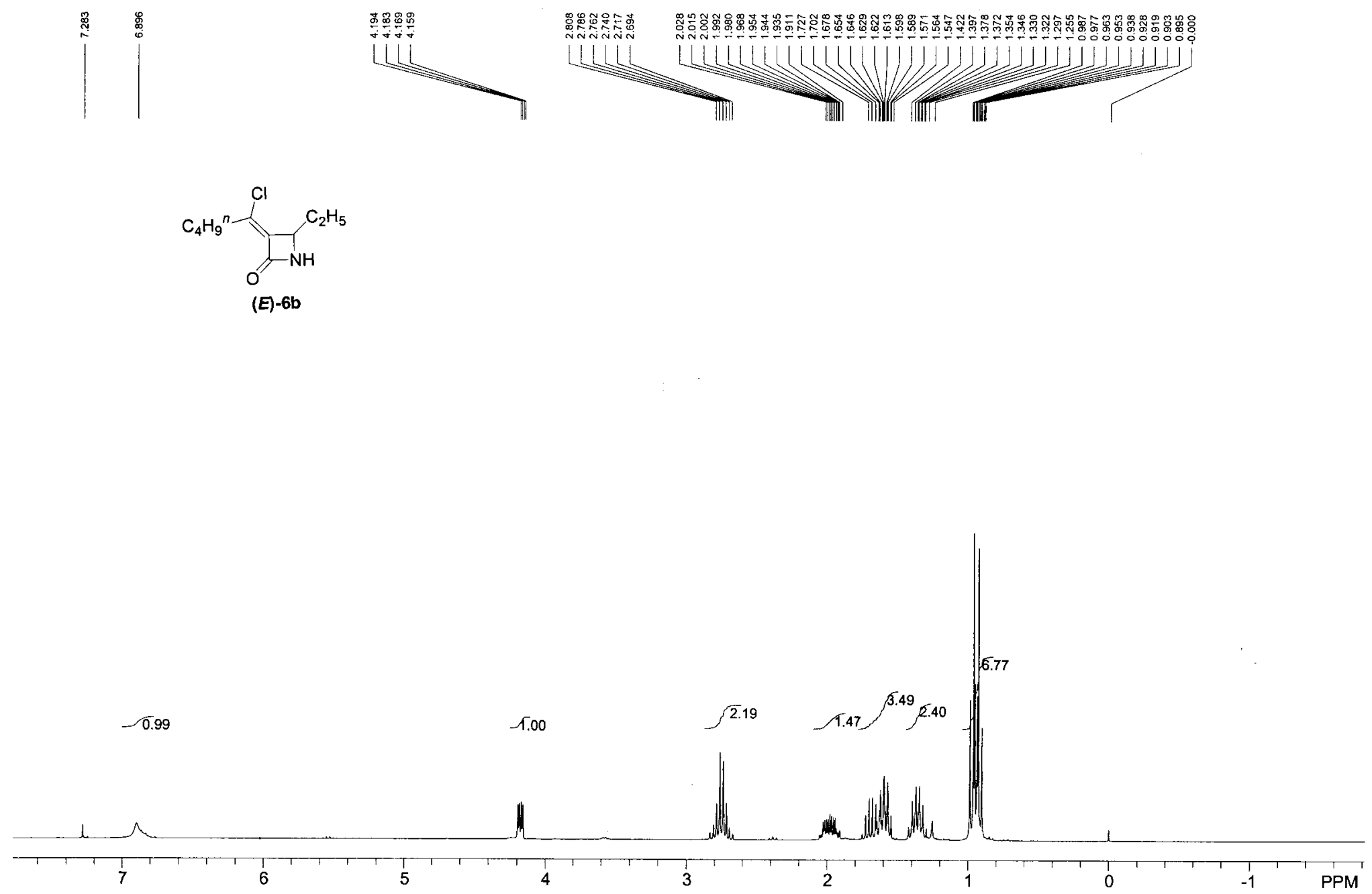

-S52- 
Archive directory: /export/home/masm/vnmrsys/data

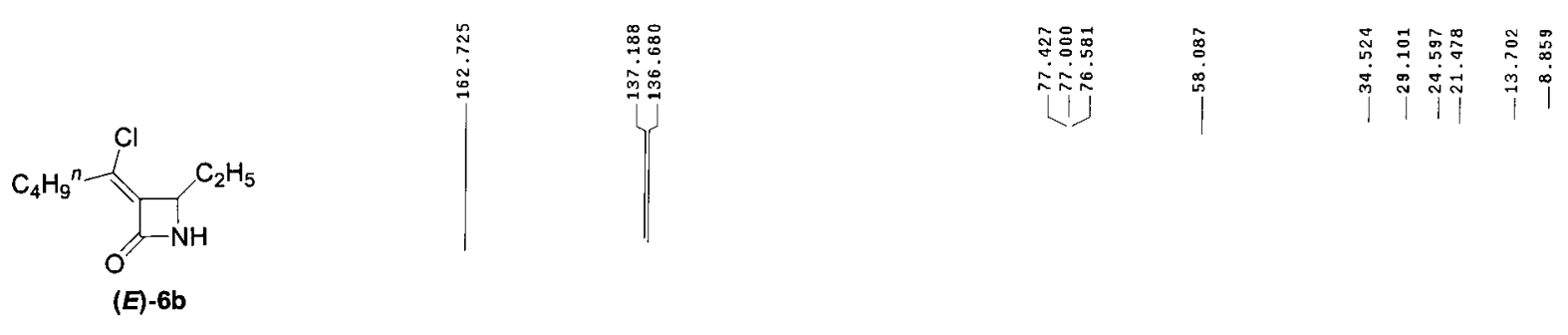

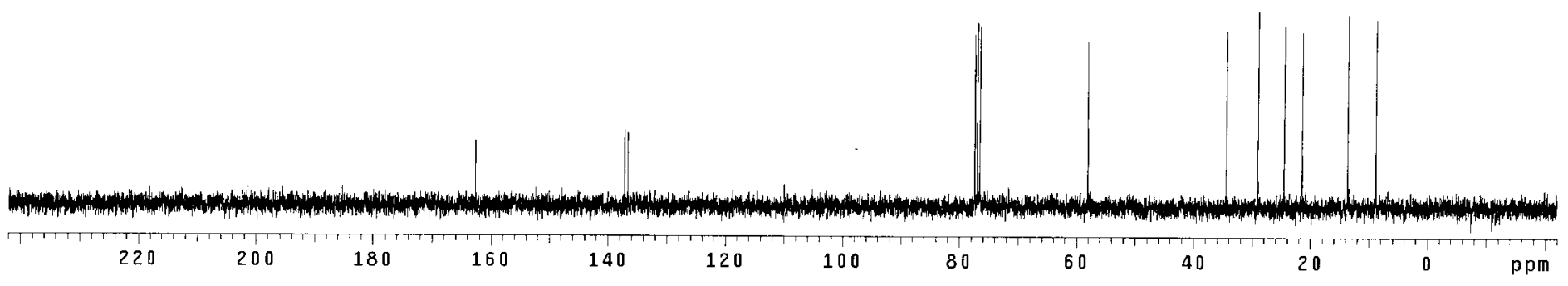



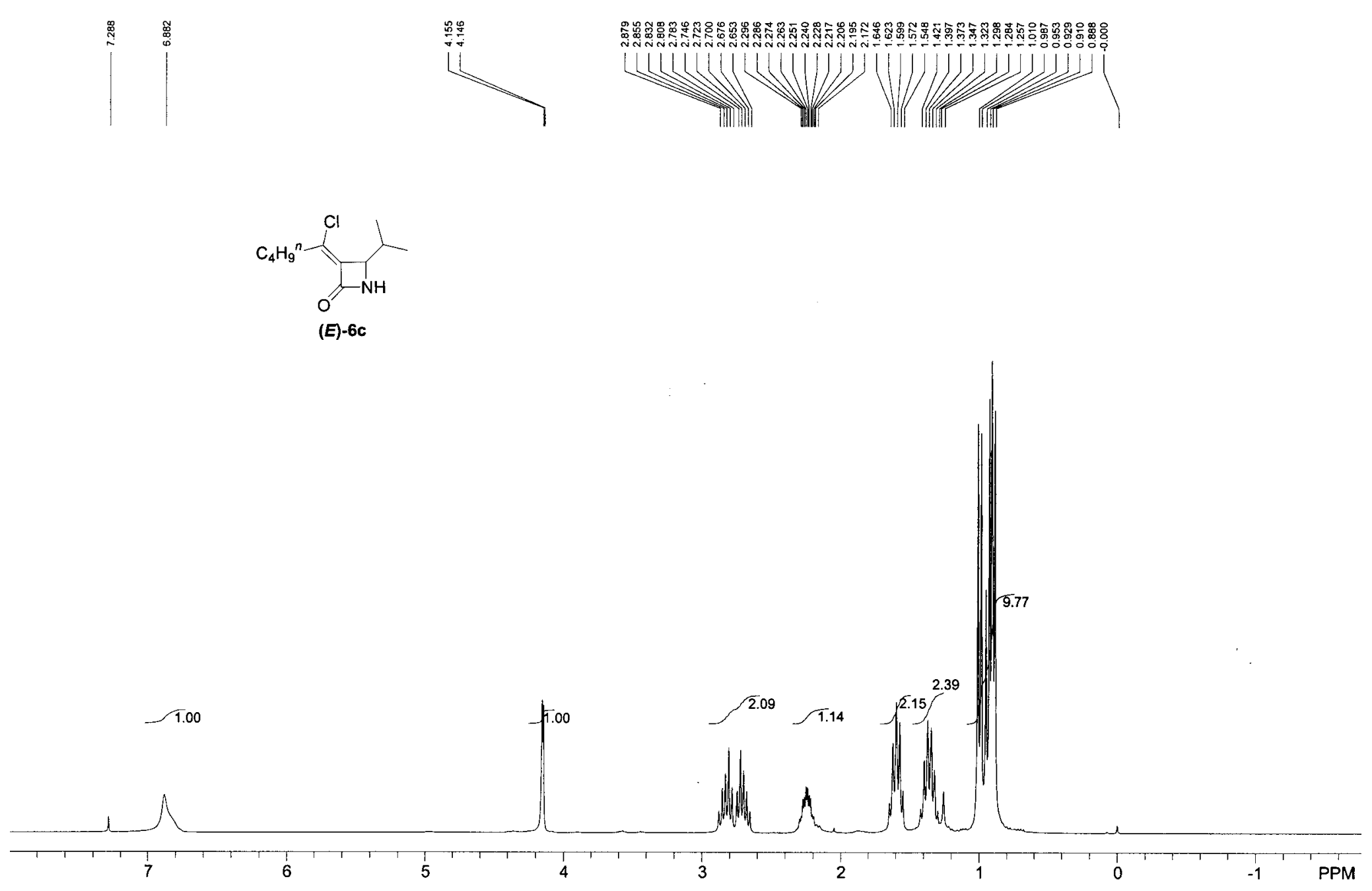
wb-8-49-c

Archive di rectory: /export/home/masm/vnmrsys/data

directory:

Pulse Sequence: szpu
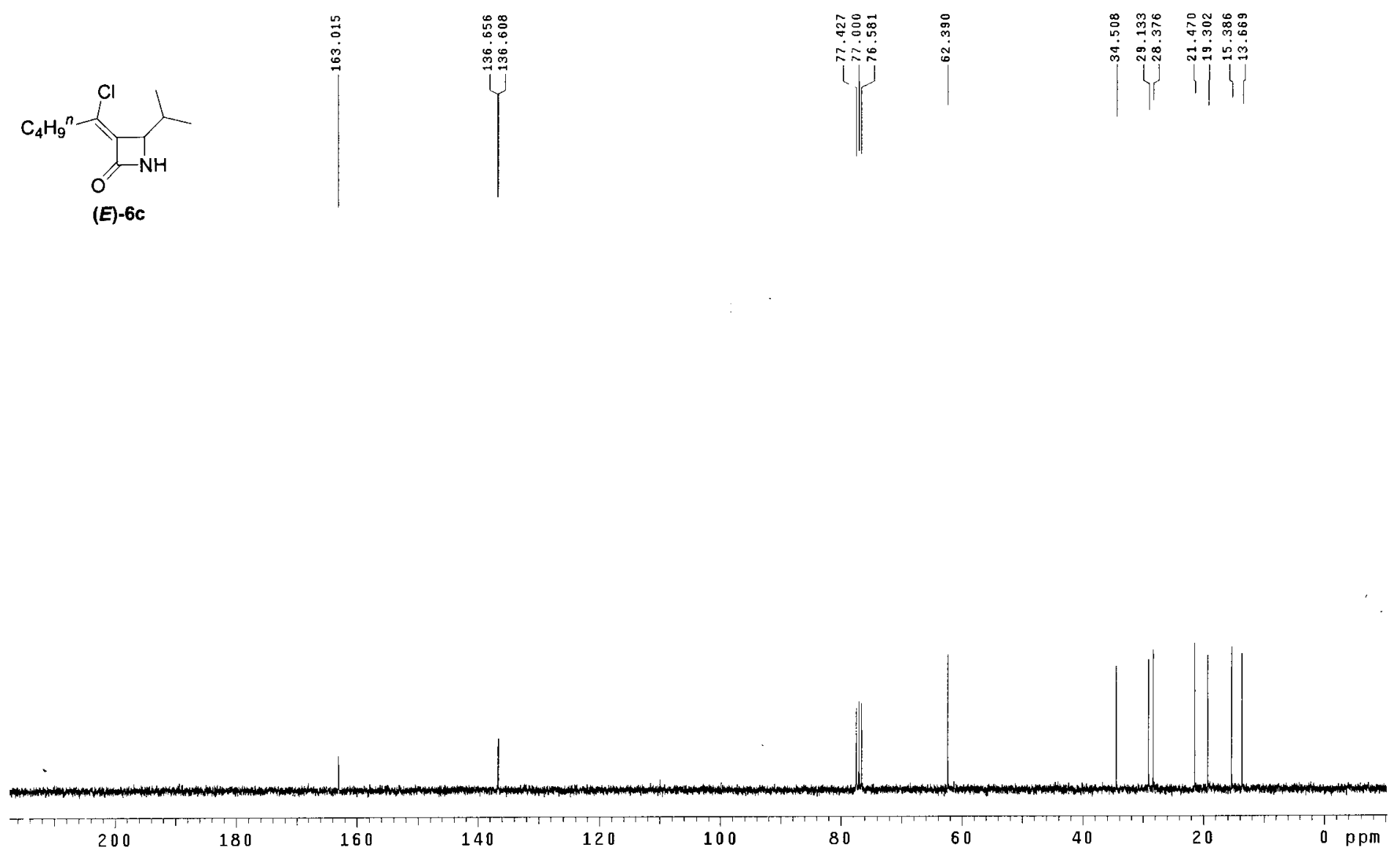

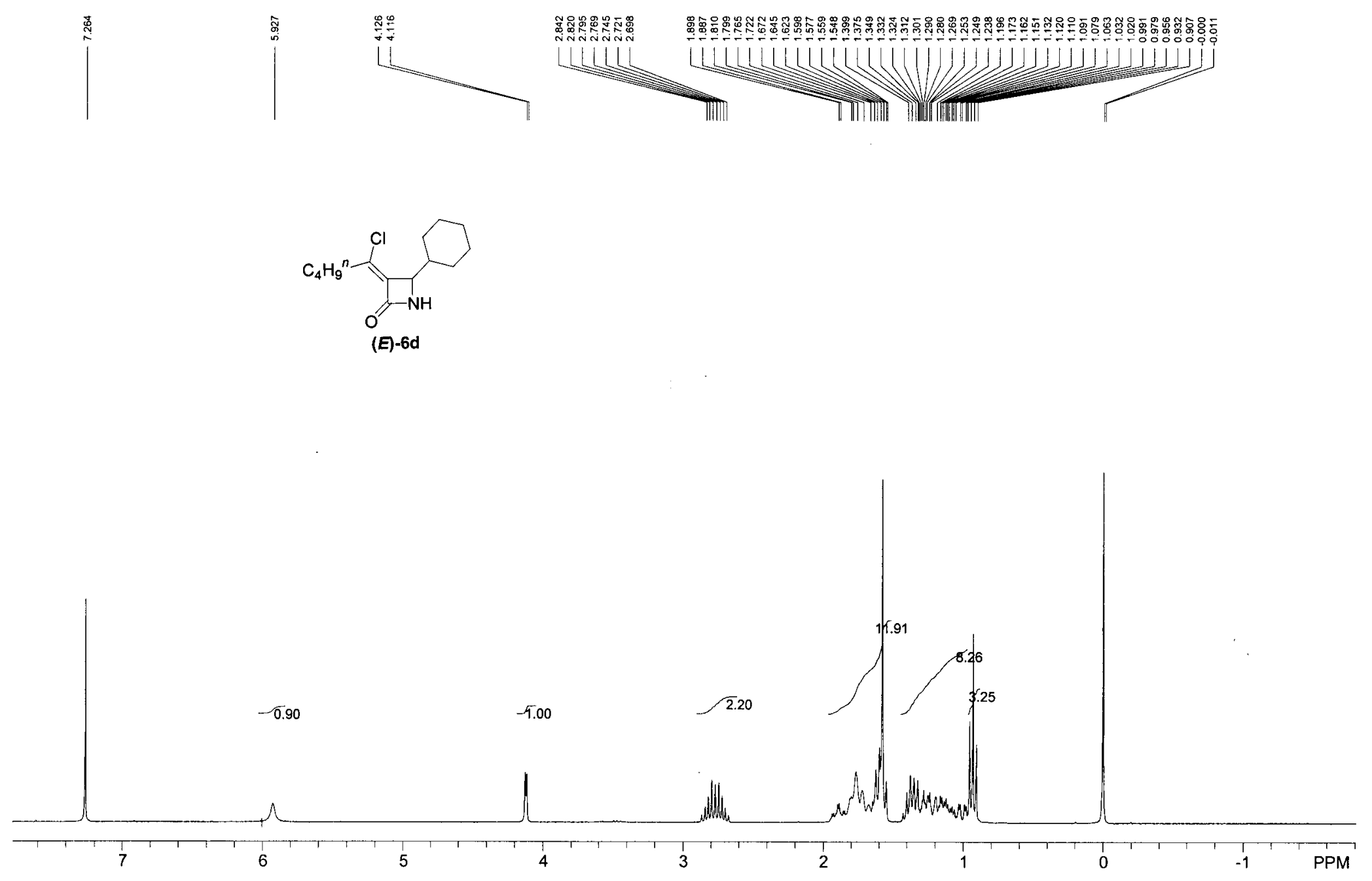
b-8-52-c

Archive directory: /export/home/masm/unnrsys/dat

directory
ARBON

Pulse Sequence: 52pur
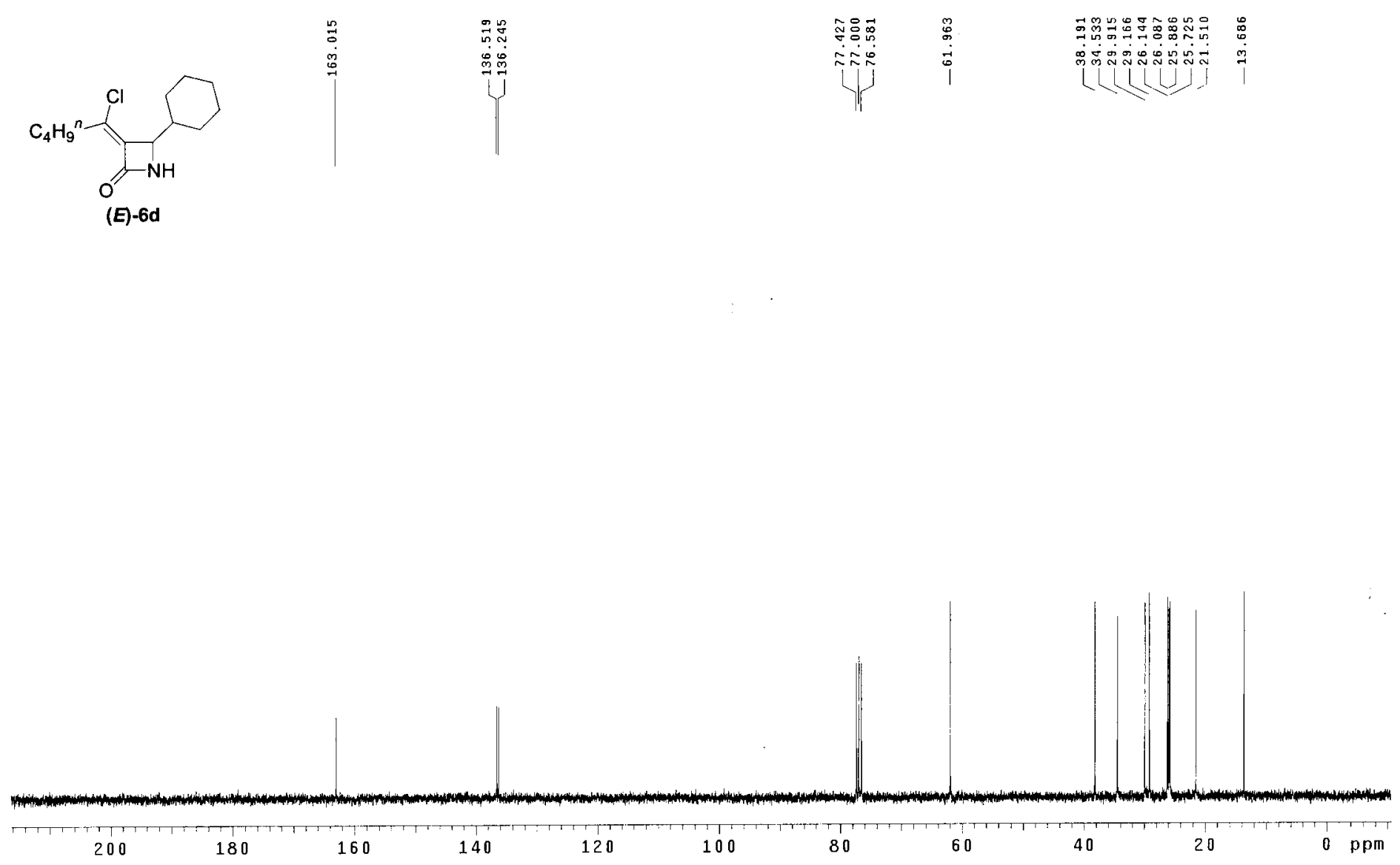


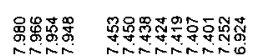

Why

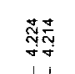

4

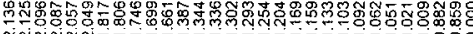

(1)

y y Yylfos
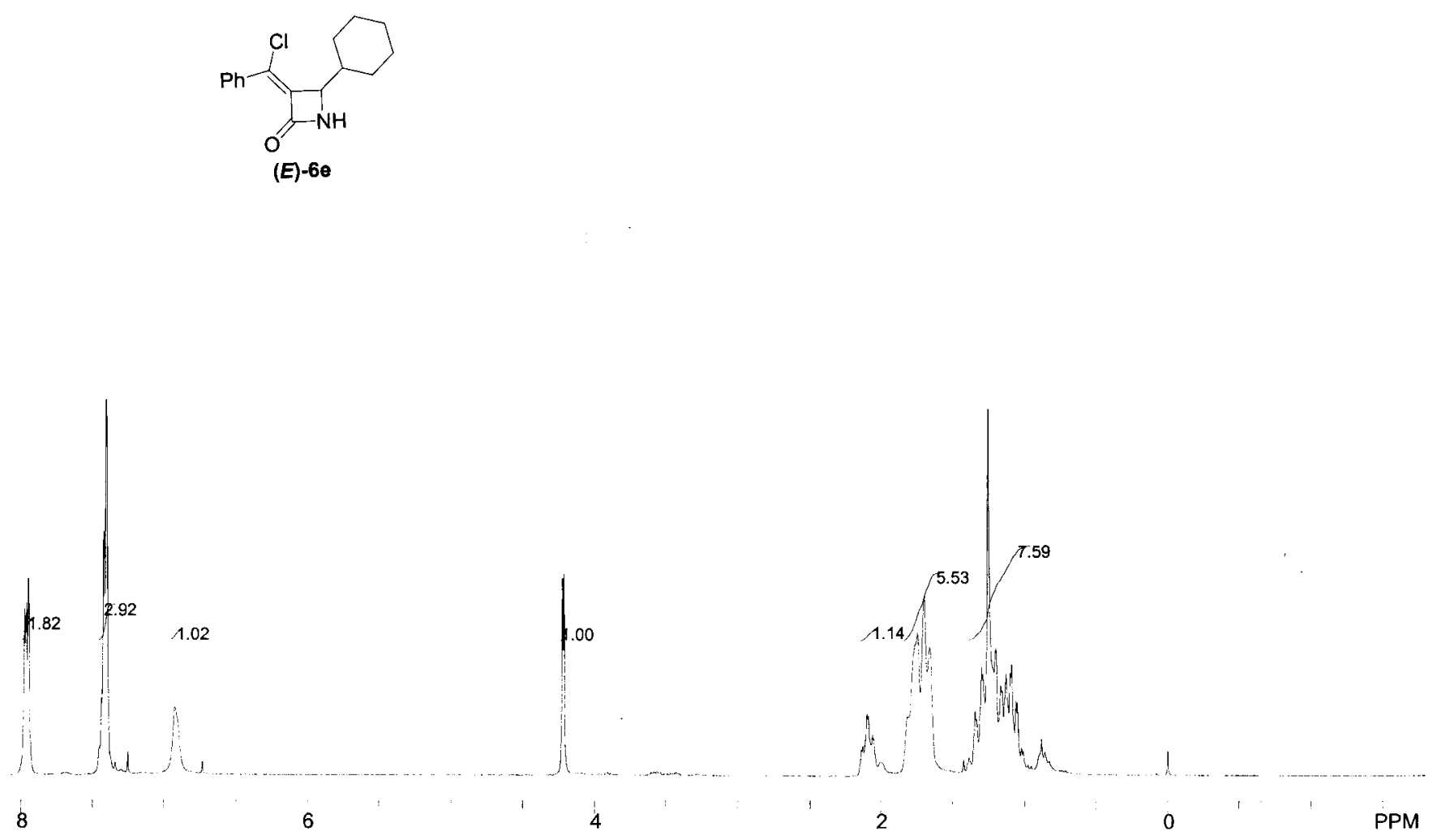

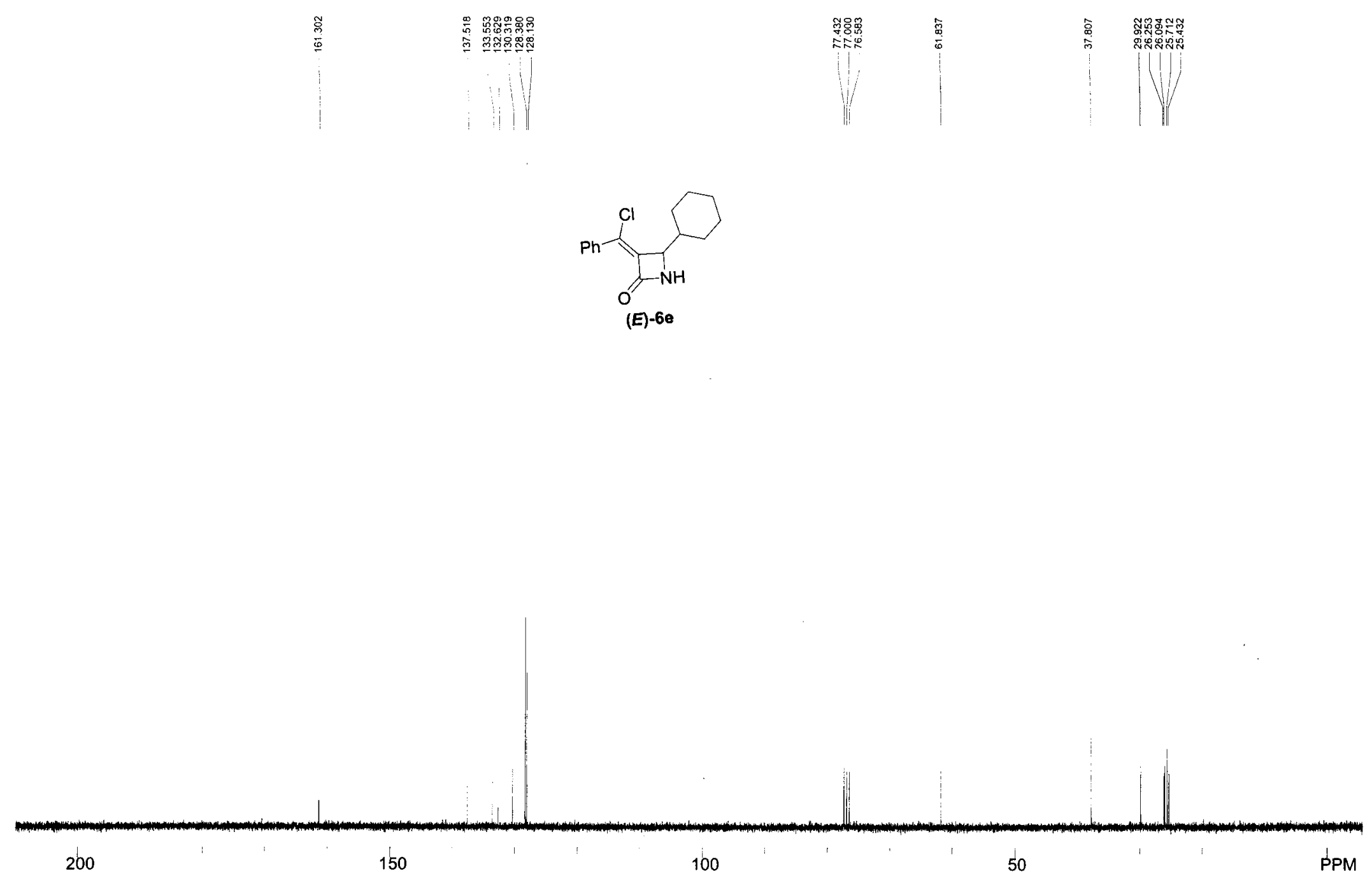

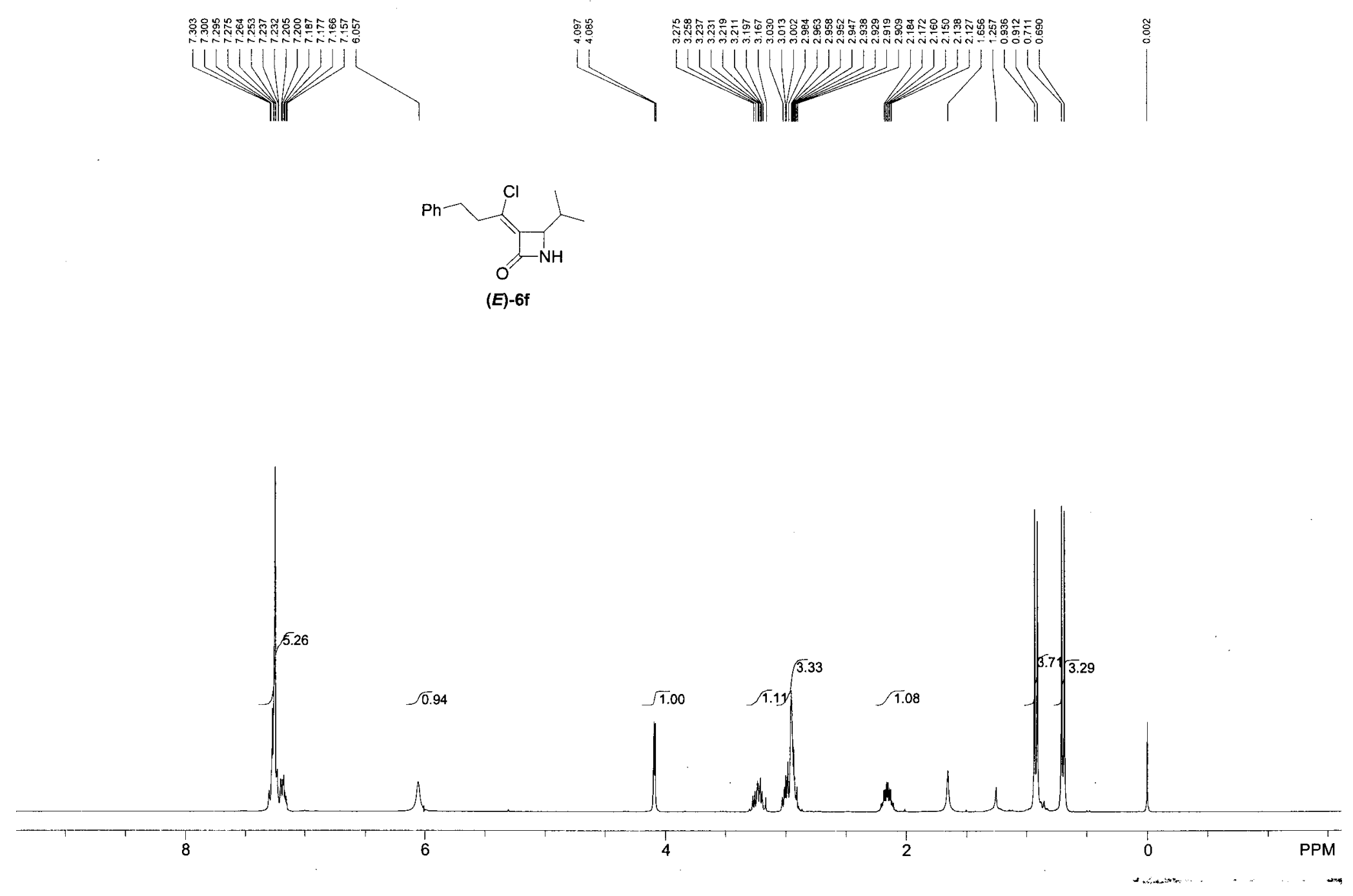
Ab-8-65-c

Archive directory: /export/home/masm/vnmrsys/data

e directory

Pulse Sequence: s2pur
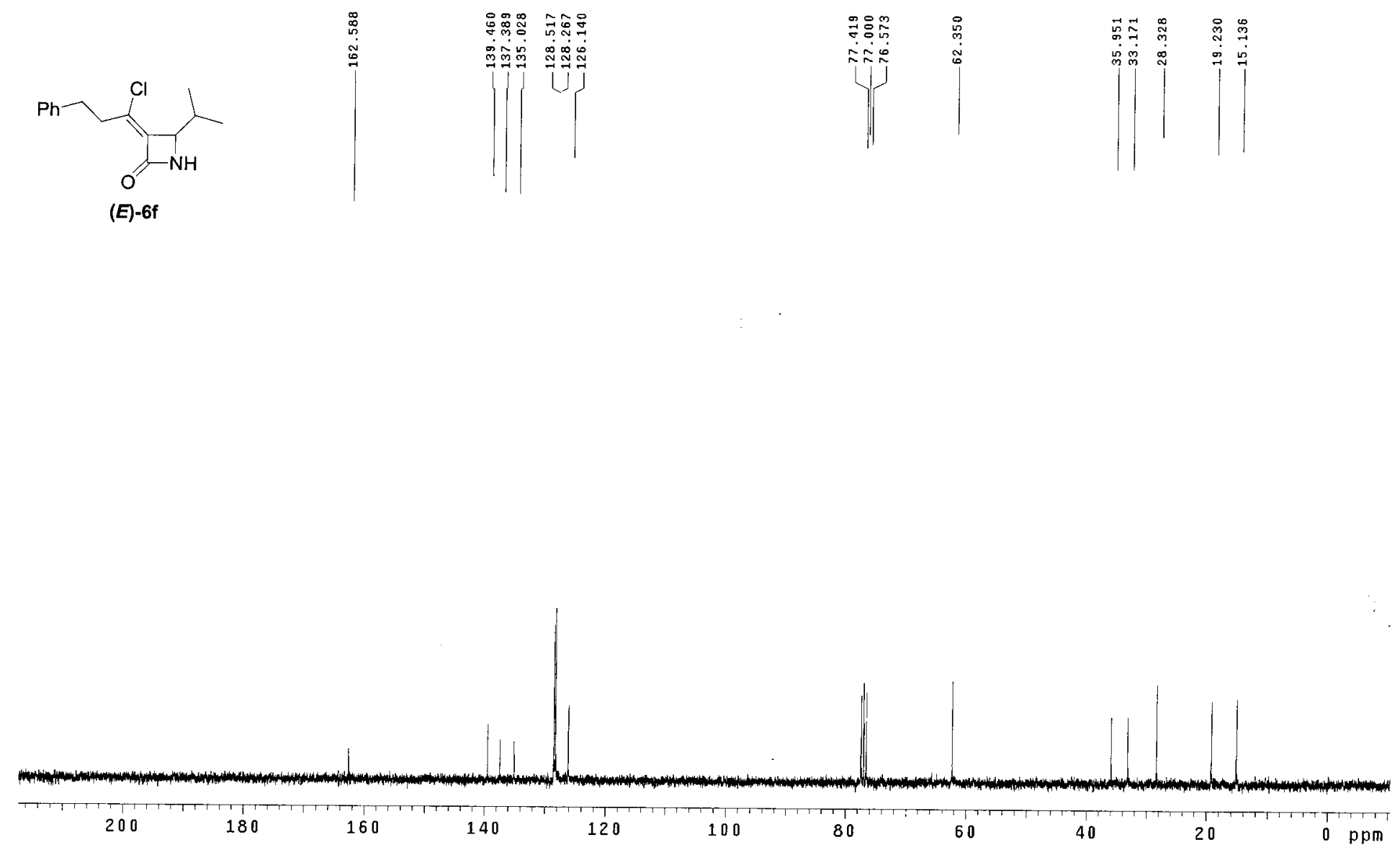

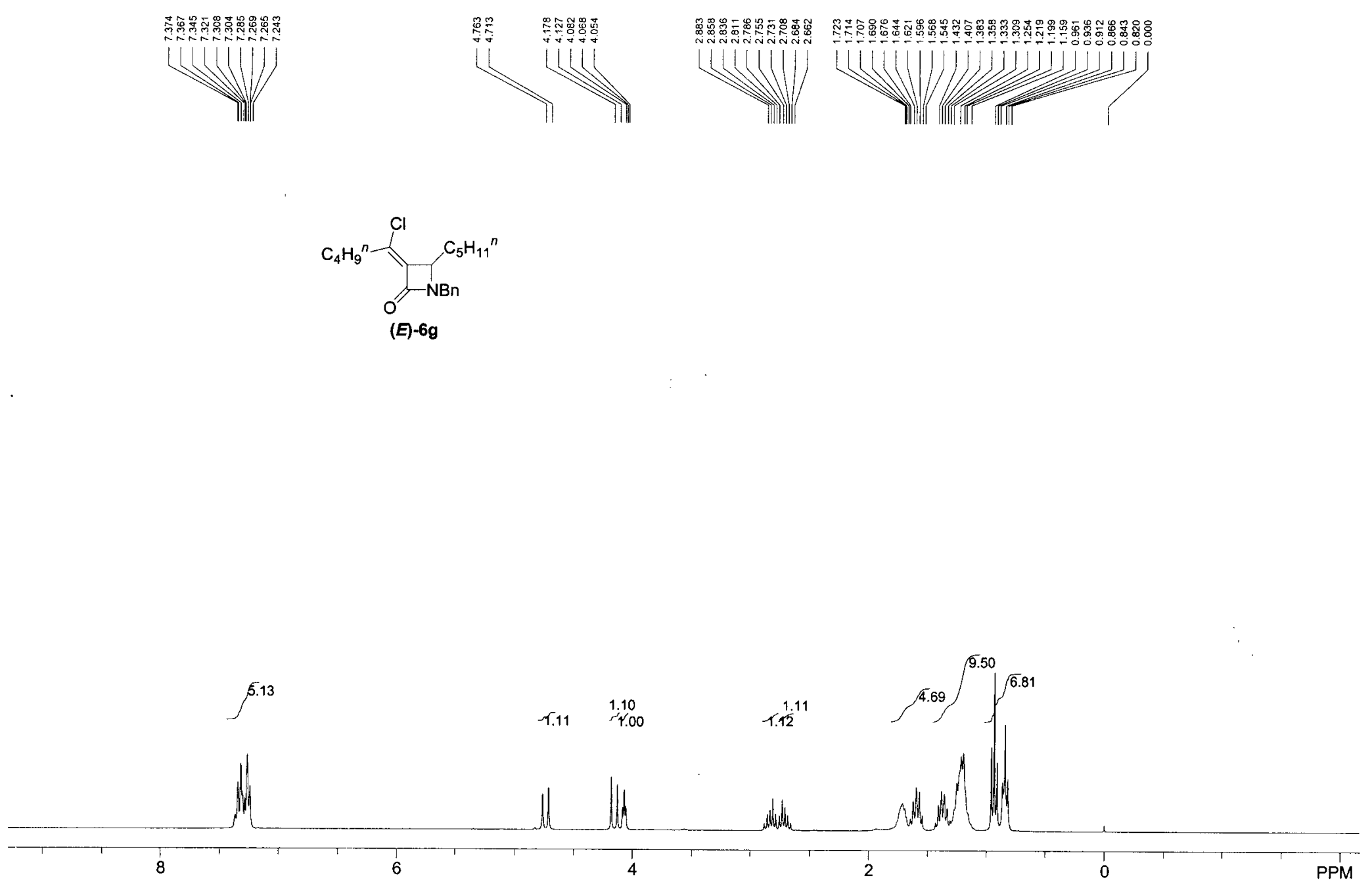
wb-8-76-c

Archive directory: /export/home/masm/ynarsys/dat

Sile: CARBON

Pu 1 se Sequence: s2pul
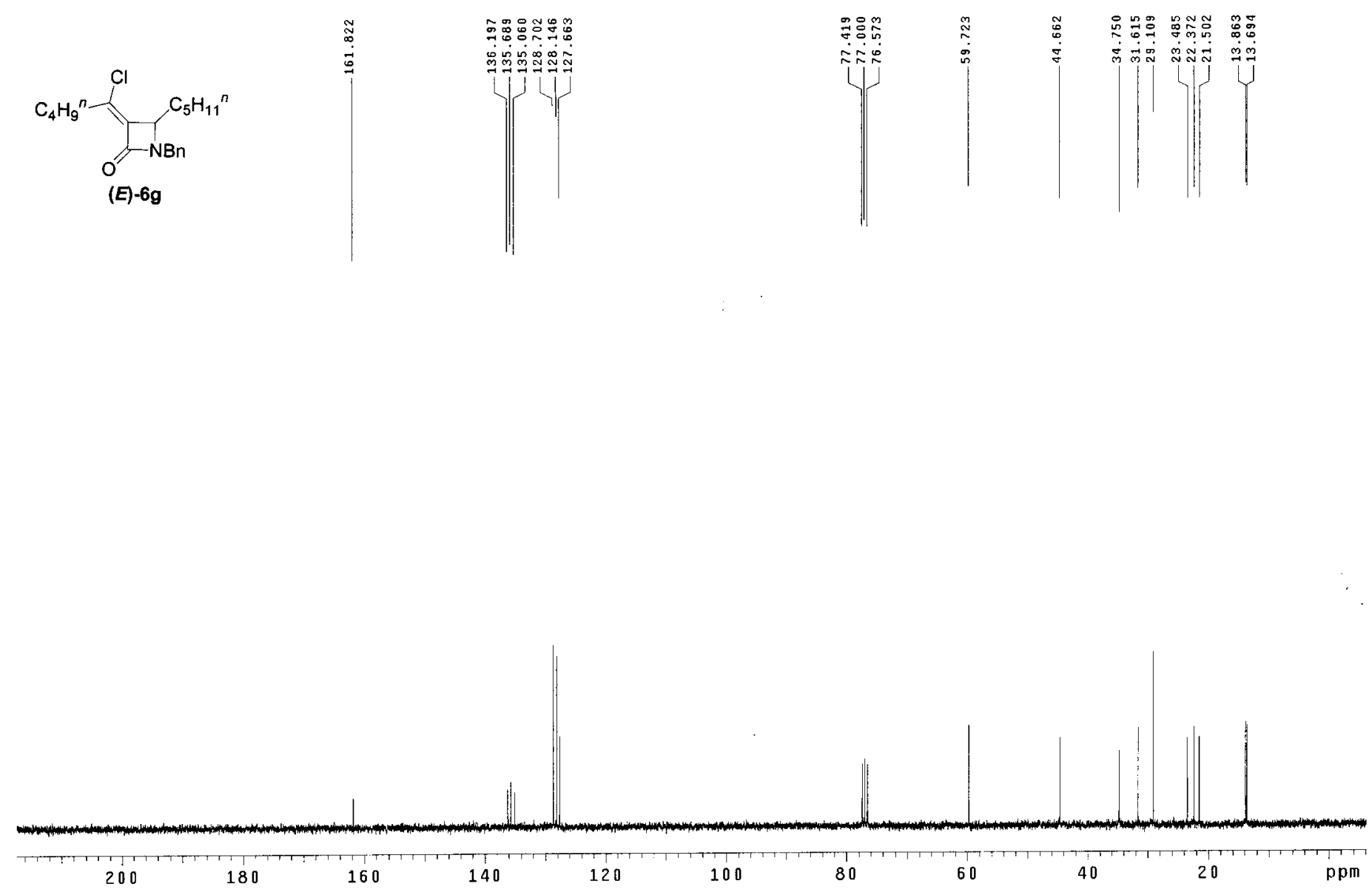

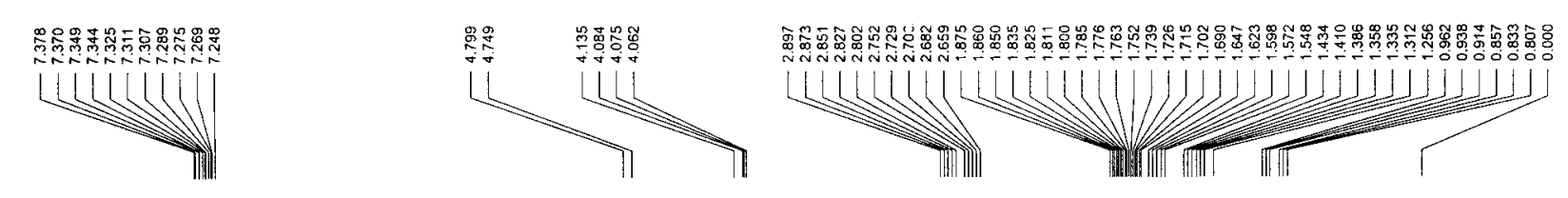

$$
\mathrm{N}_{(E)-6 \mathrm{~h}}^{\mathrm{C}_{2} \mathrm{H}_{5}}
$$

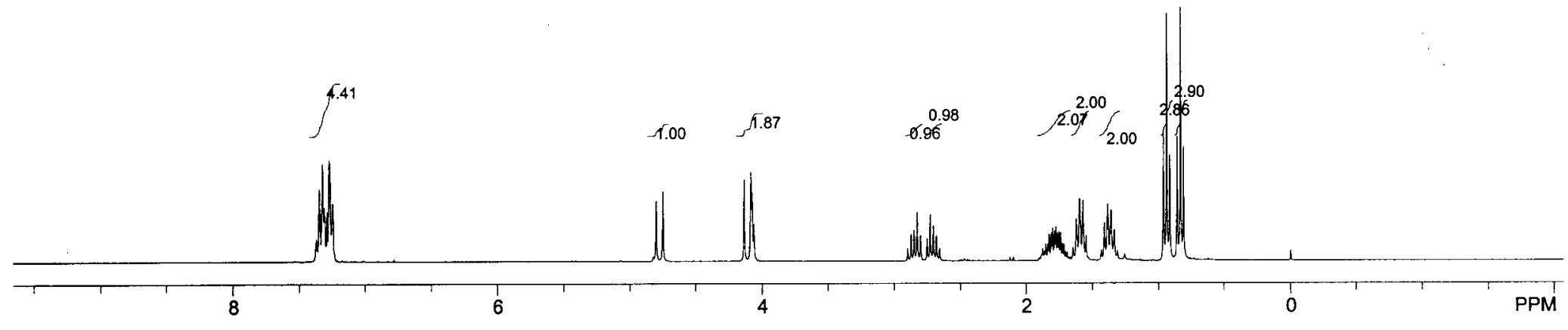


Archive di rectory: /export/home/masm/vnmrsys/data

tive directory:

Pulse sequence: s2pul
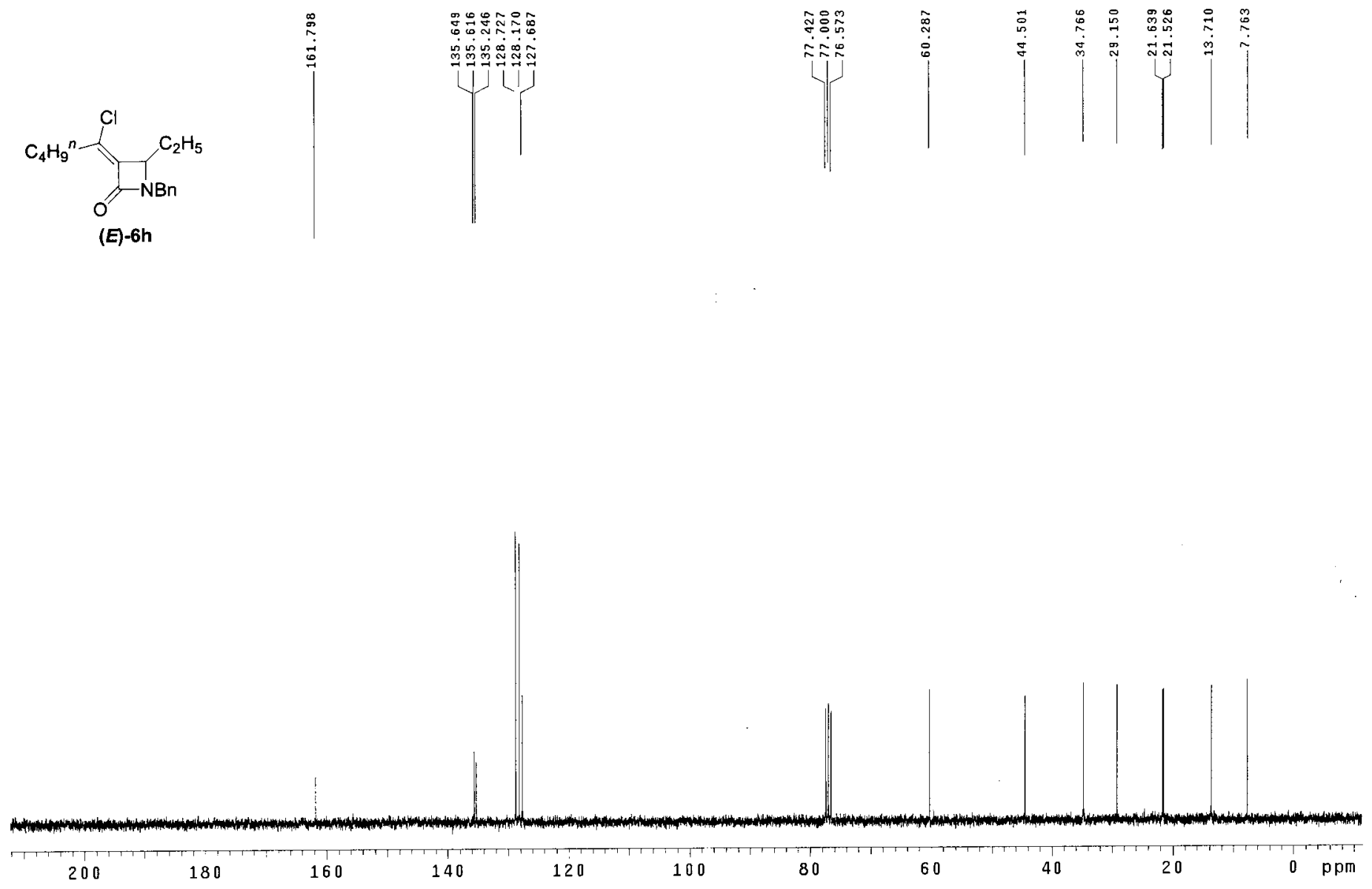

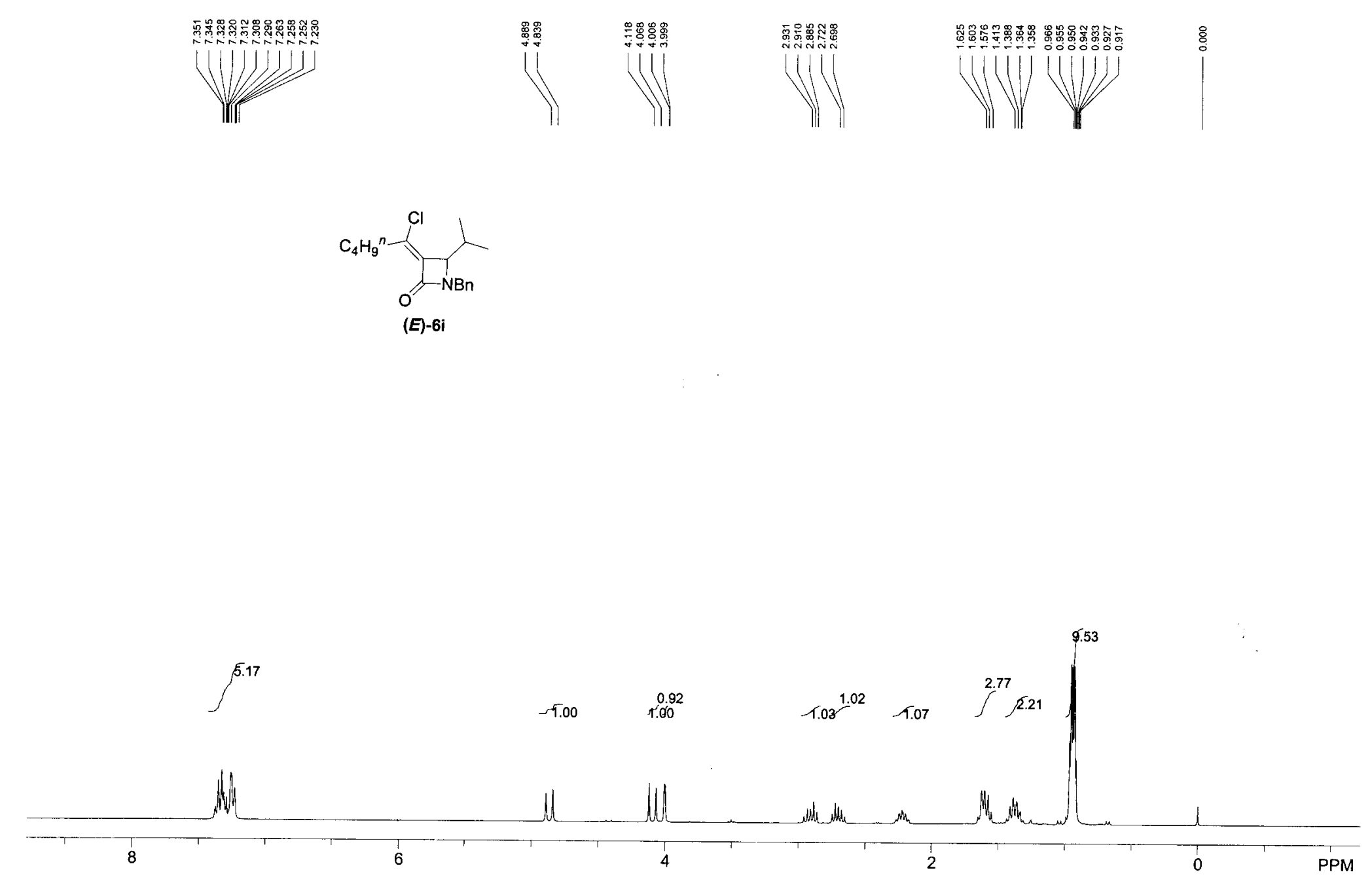
wb-8-61-c13

Arenive directory: /export/home/masm/unmrsys/data

Sample directory
File: CARBON

Pulse Sequence: s2pul
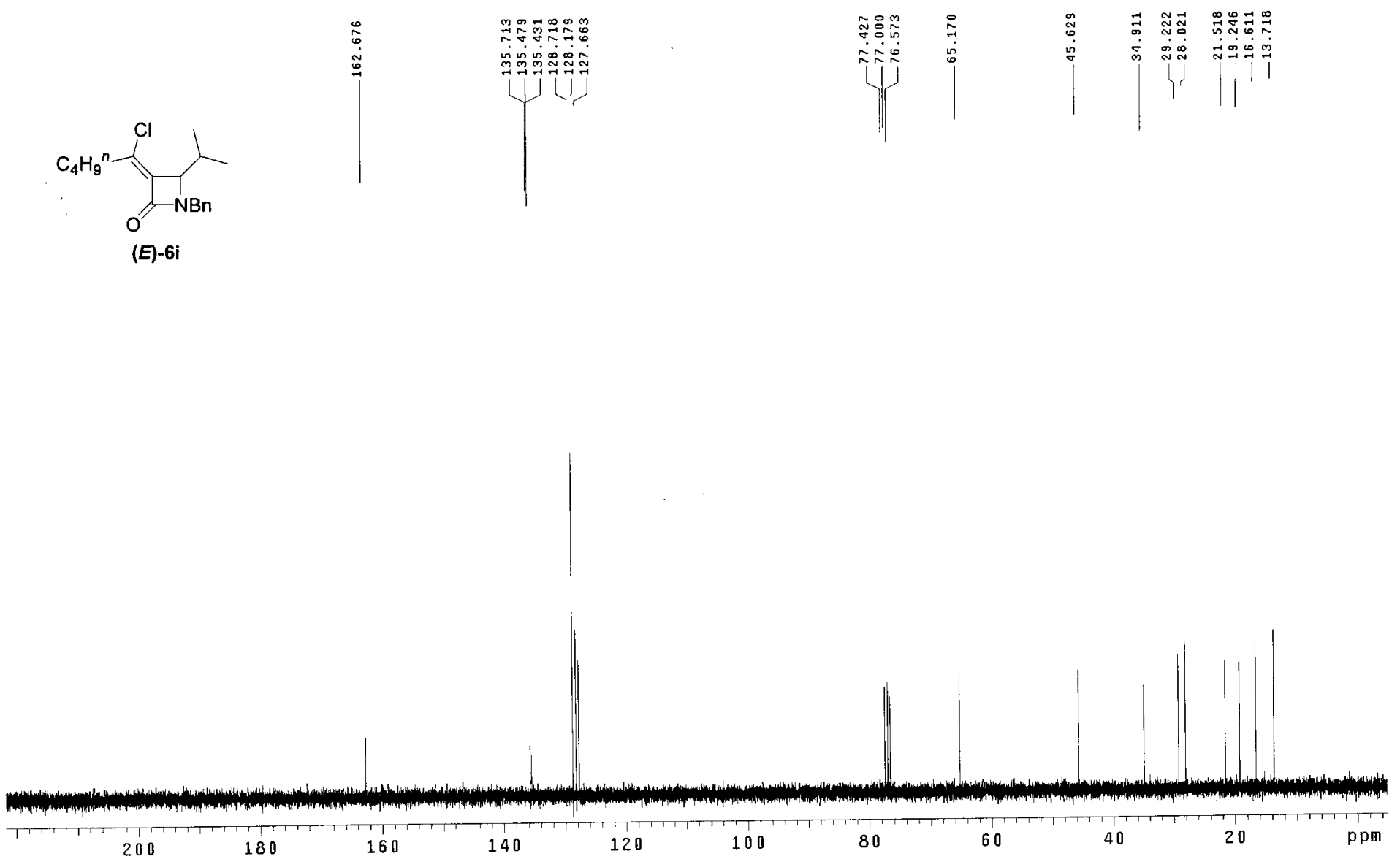

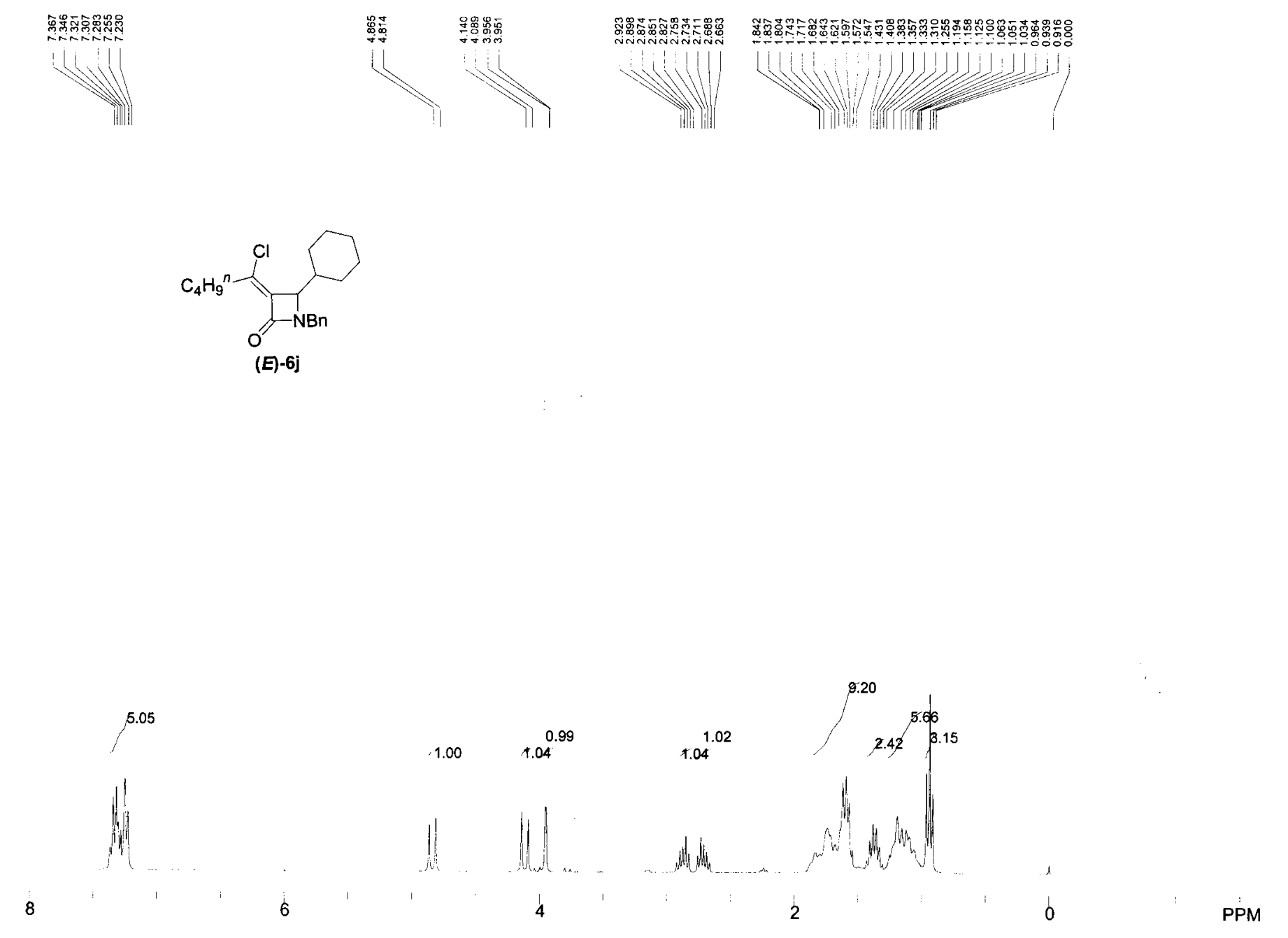


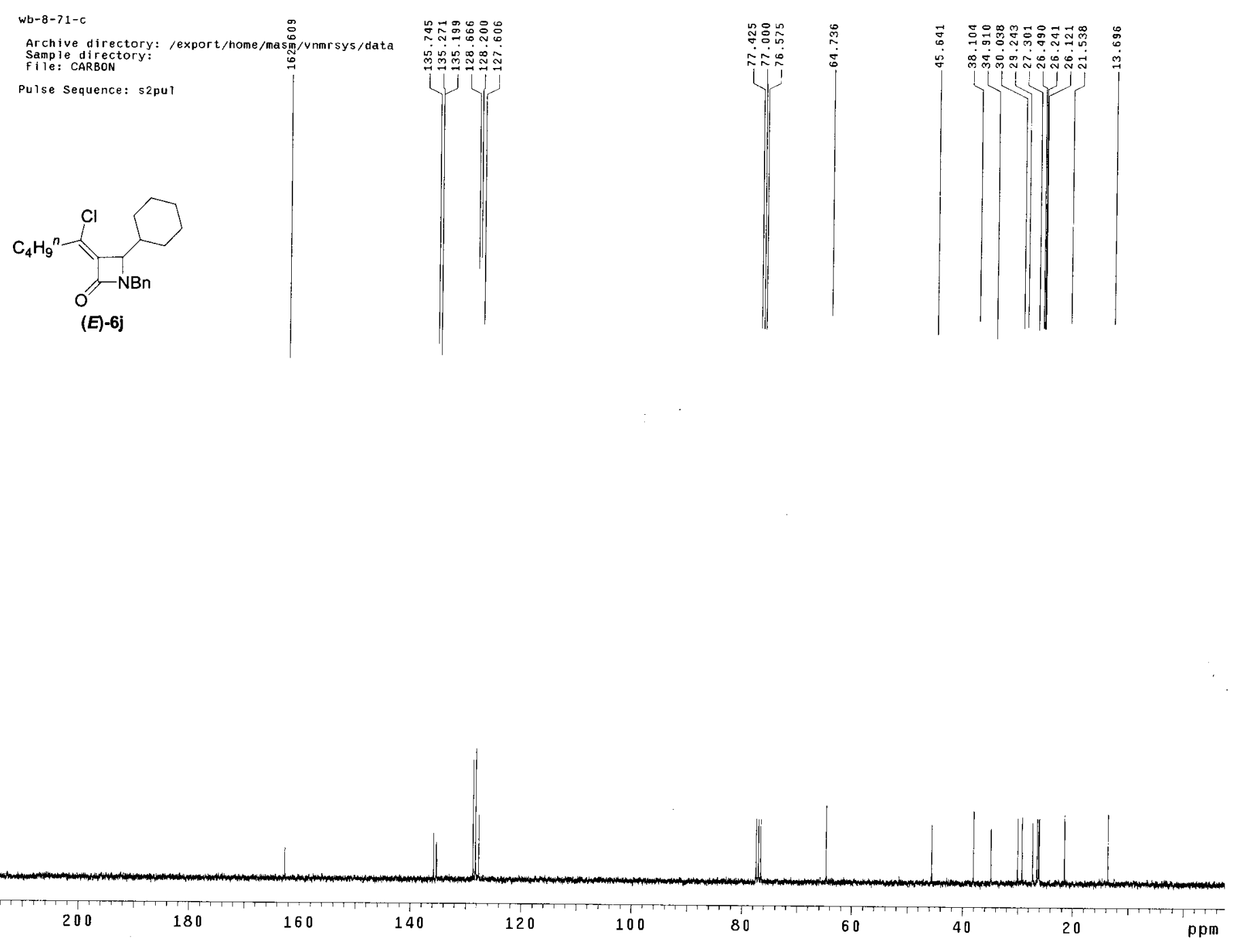



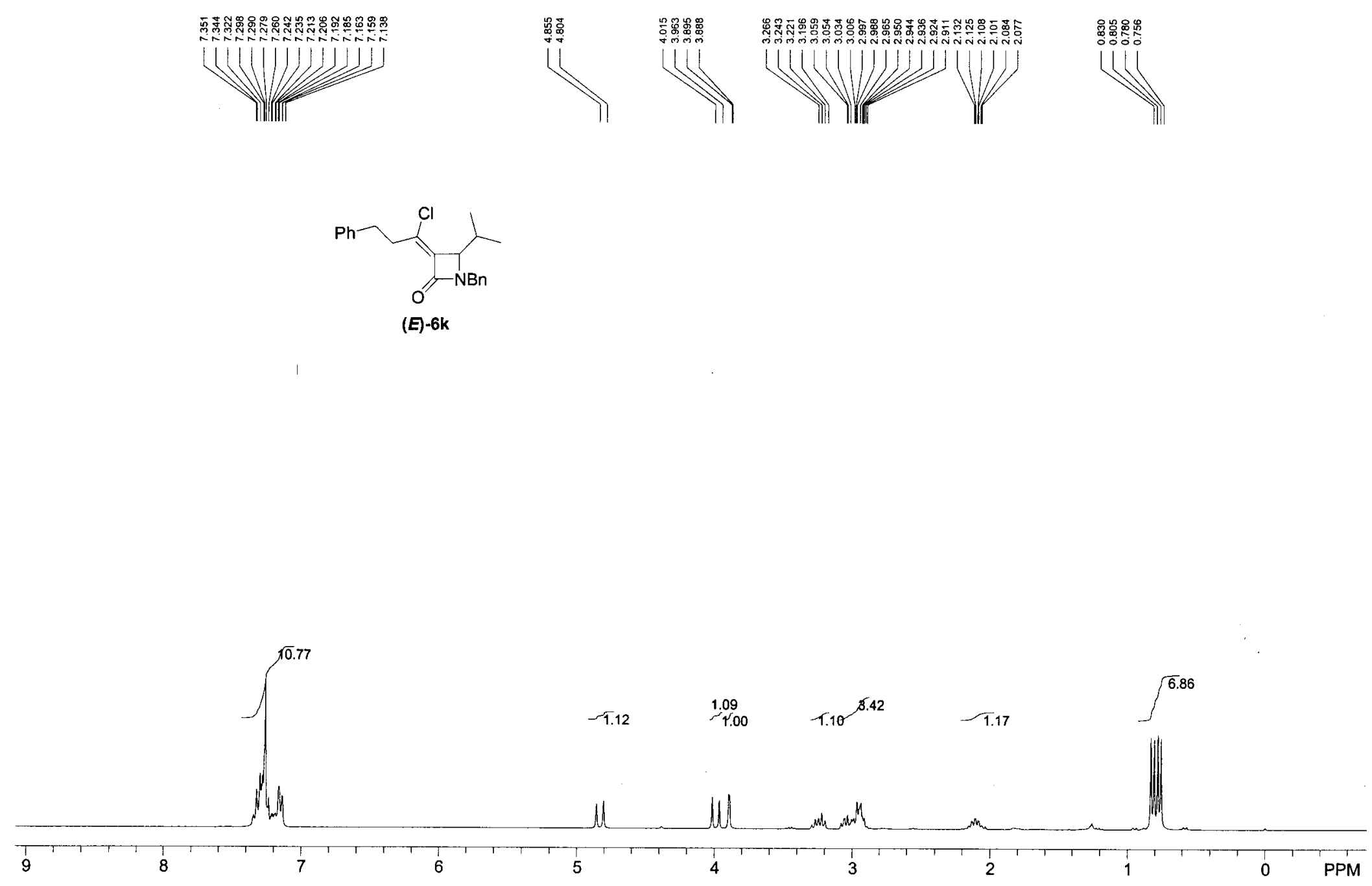
wb-8-77-c

Archive directory: /export/home/masm/vnmrsys/data
Sample directory:

Sample directory
file: CARBON

Pulse Sequence: s2pul
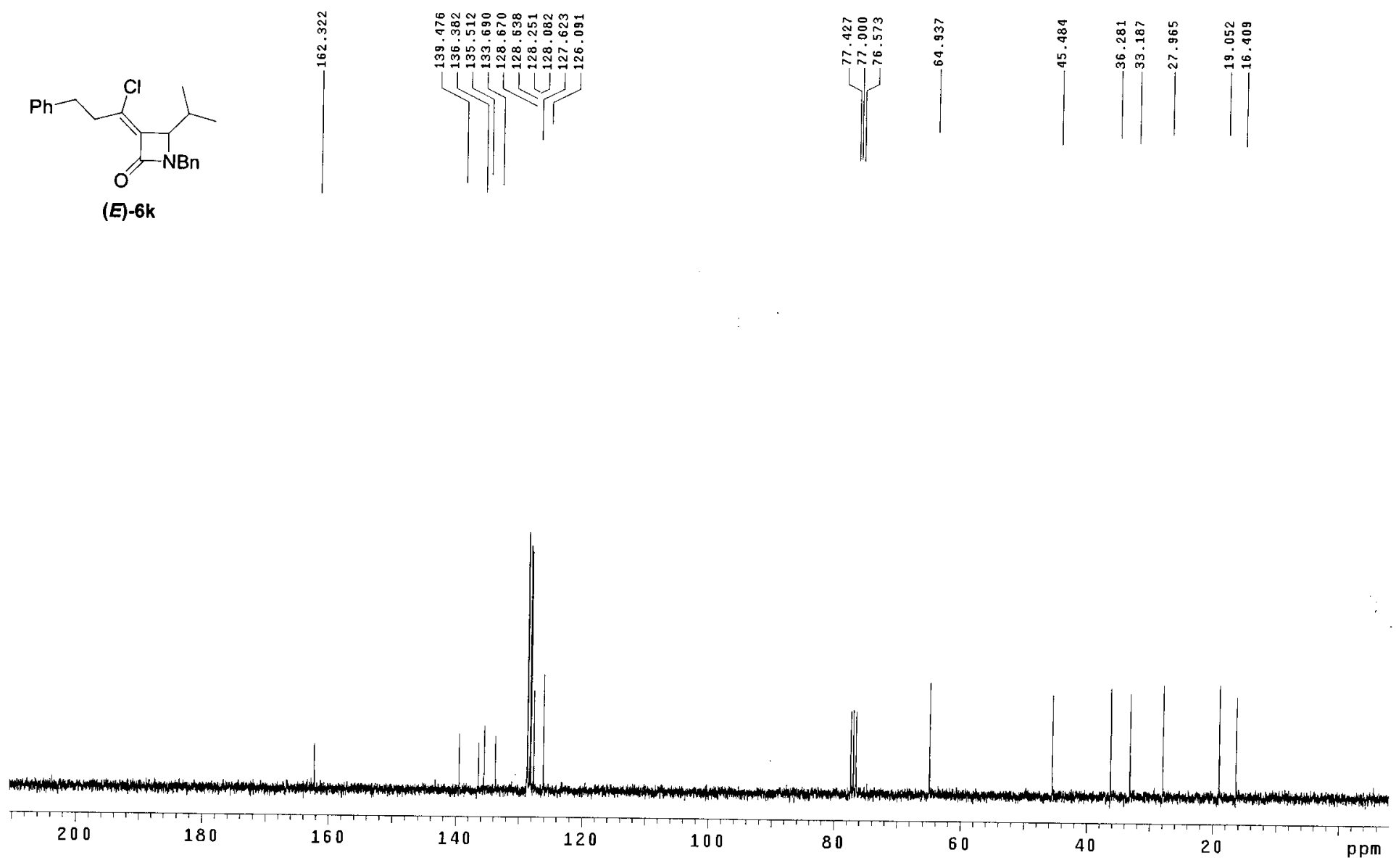


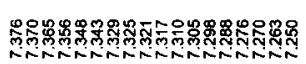

WWWULDLD!

III

กำ

4 W

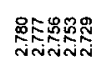

y
产

-

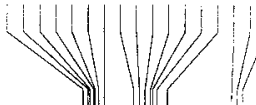

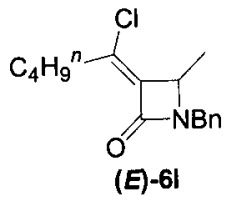

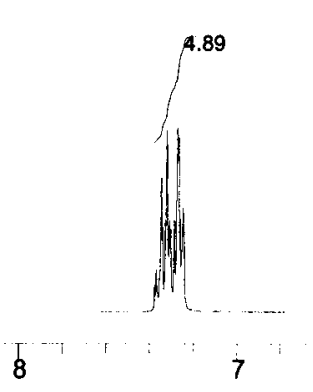

4.89
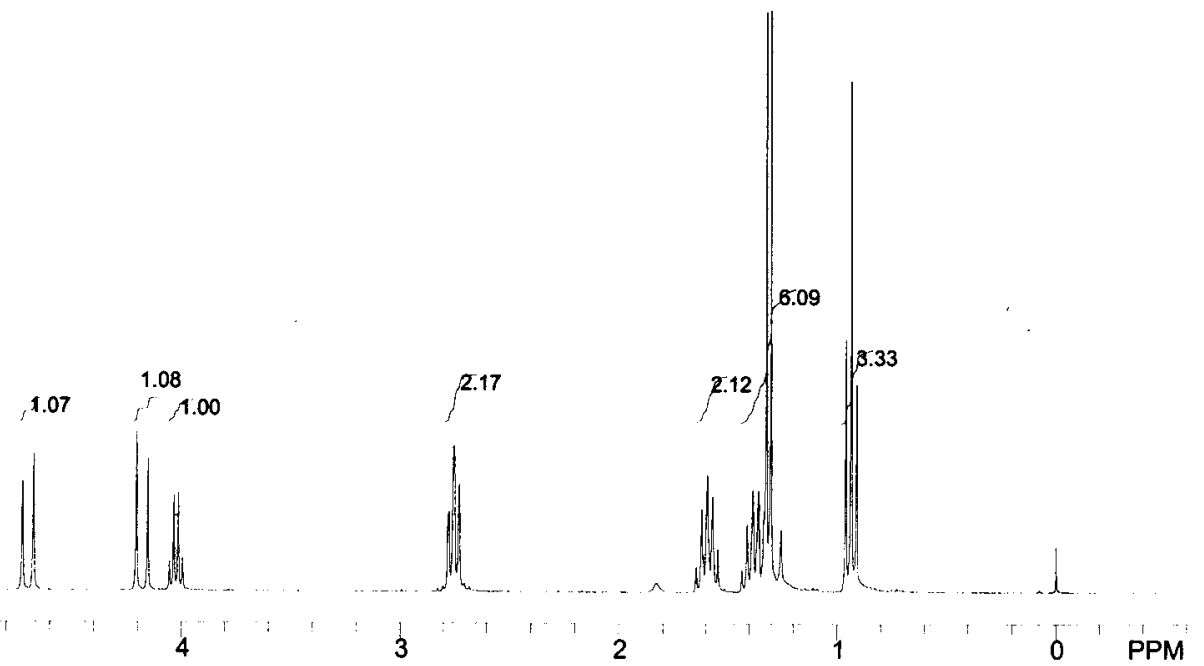
wb-7-199-c

Archive directory: /export/home/masm/vnmrsys/data

File: CARBON

Pulse Sequence: s2ou
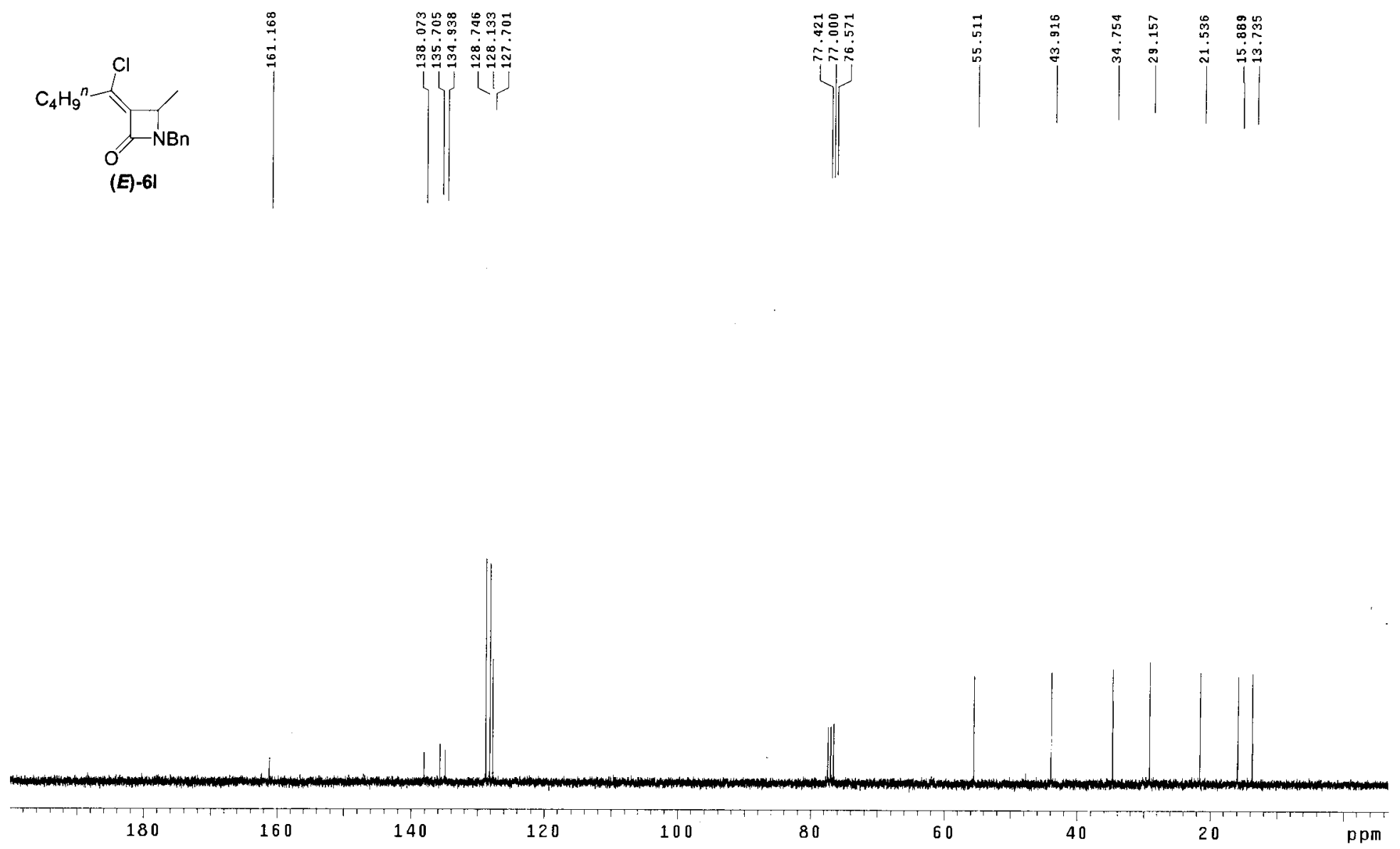


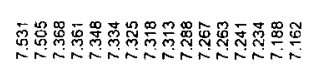

[Huluty

管

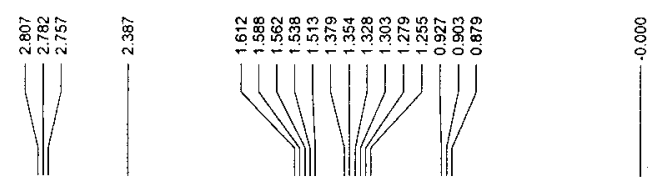
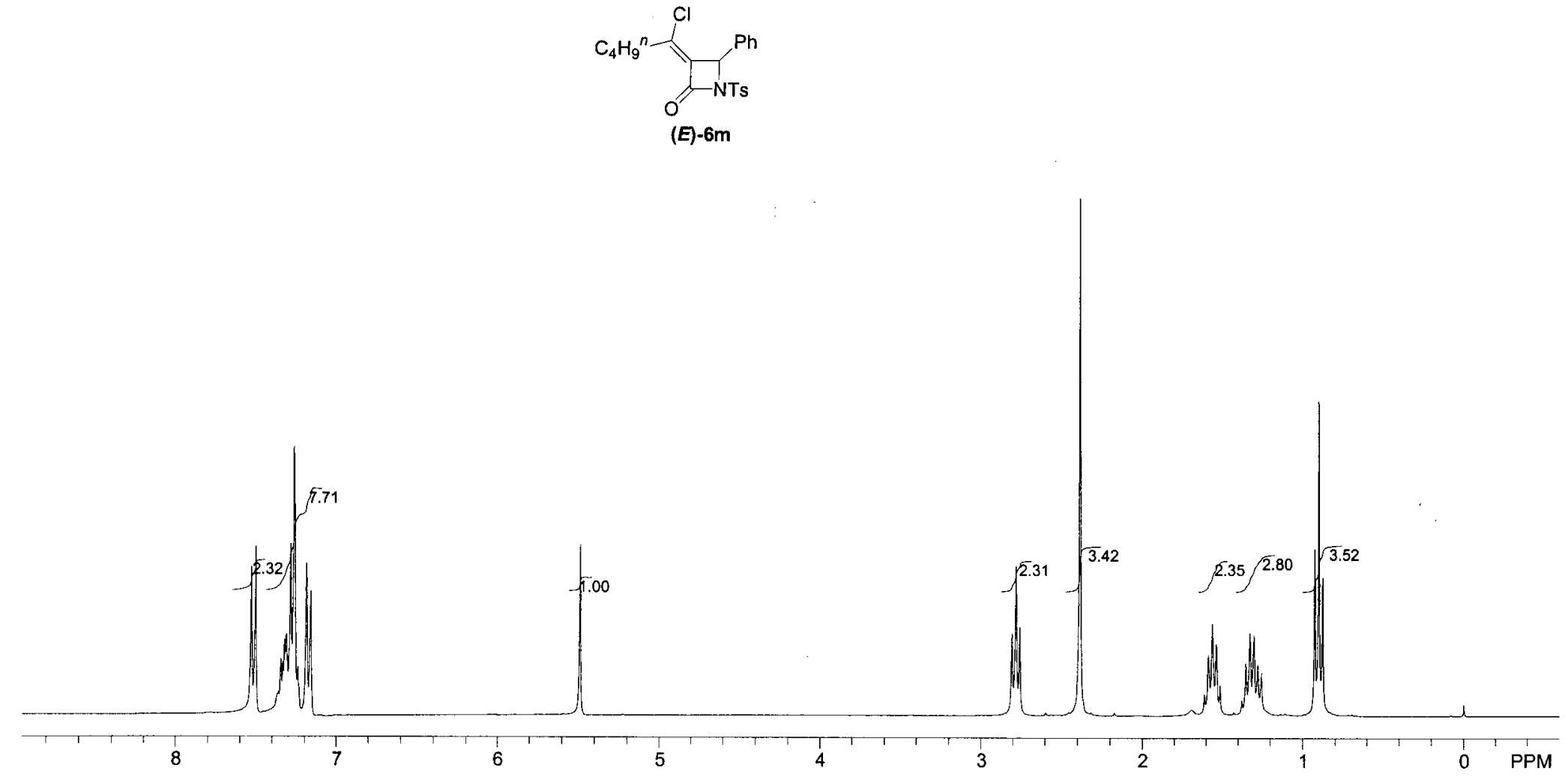
wb-8-81-c

Archive directory: /export/home/masm/vnmirsys/data

File: CARBON

Pulse Sequence: $52 \mathrm{pul}$

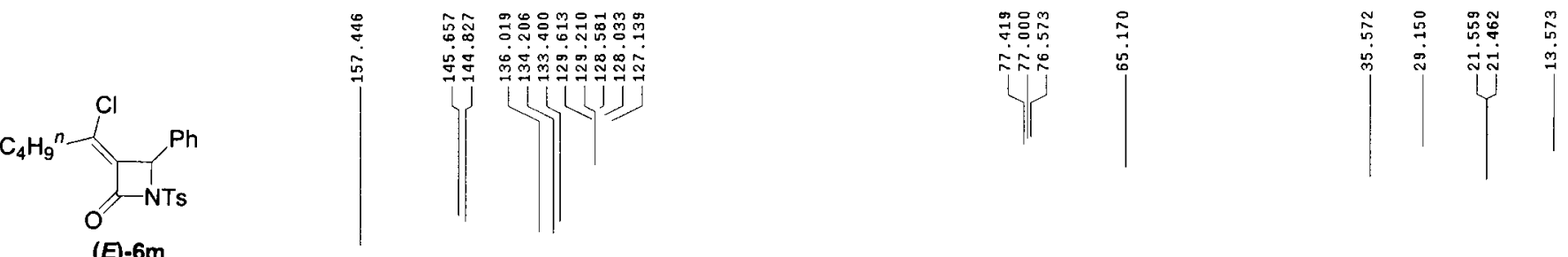

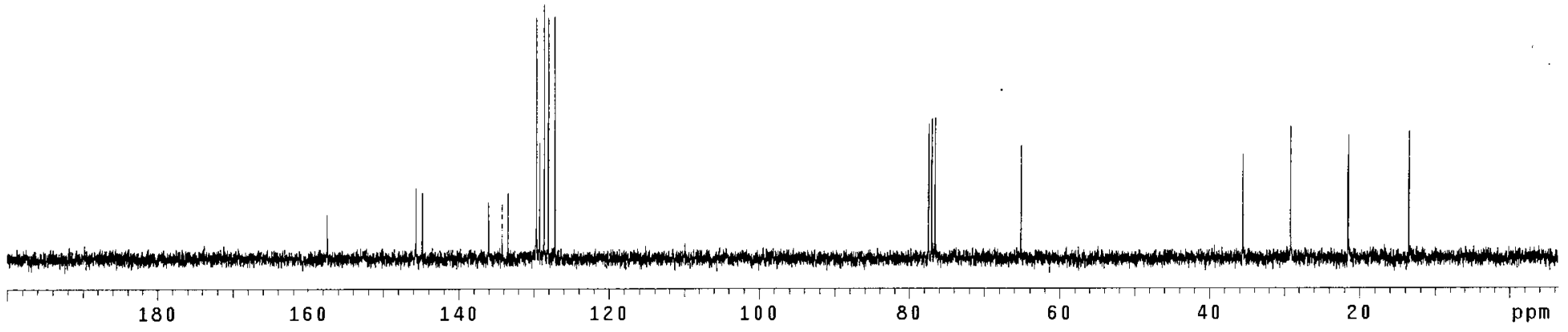

